\title{
tracione \\ Stratigraphic Sections of the \\ Phosphoria Formation in \\ Idaho, 1949, Part I
}

By R. P. Sheldon, M. A. Warner, M. E. Thompson, and H. W. Peirce
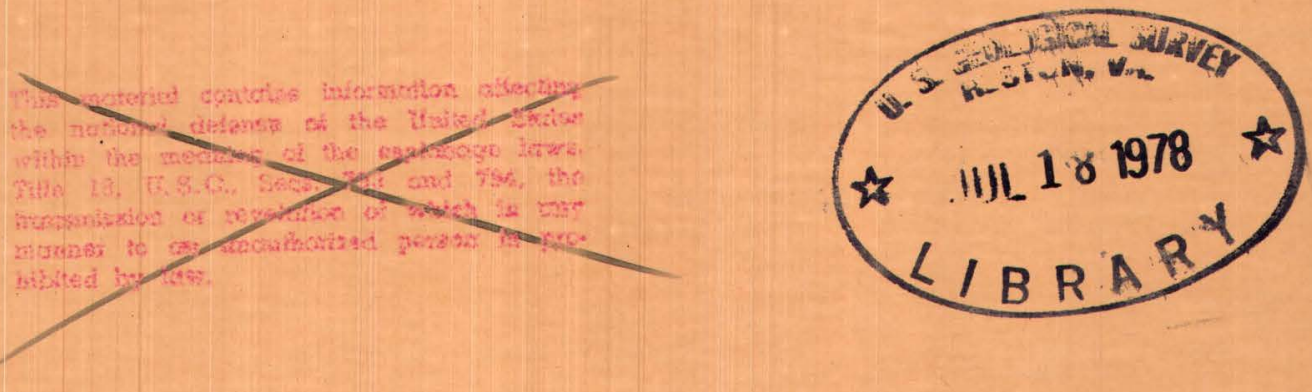

\section{Trace Elements Investigations Report 364}

UNITED STATES DEPARTMENT OF THE INTERIOR

US, GEOLOGICAL SURVEY

Declassified $3 / 10 / 69$ 
Geology and Mineralogy

This document consists of 36 pages. Series A

UNITED STATES DEPARTMENT OF THE INTERIOR

$\checkmark$ U.S. GEOLOGICAL SURVEY

STRATIGRAPHIC SECTIONS OF THE PHOSPHORIA FORMATION

IN IDAHO, 1949, PART I*

By

R. P. Sheldon, M。 A. Warner, M。 $\mathbb{E}$ 。 Thompson, and $H_{\circ} W_{0}$ Peiree

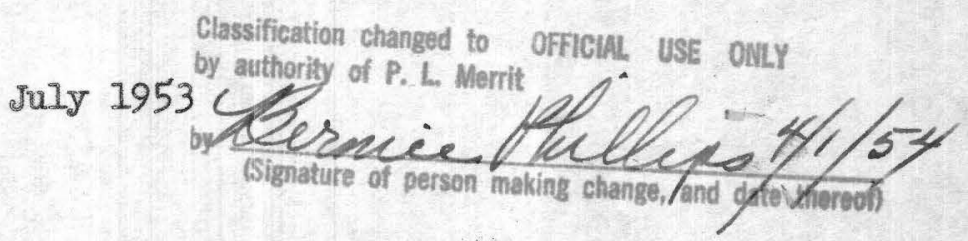

Trace Elements Investigations Report 364

This preliminary report is distributed without editorial and technical review for conformity with official standards and nomenclature. It is not for pubIic inspection or quotation.
Nis material contains information afferting the national defense of the United States within the meaning of the esplonage laws, Title 18 , U.S.C., Secs. 793 and 794 , the transmission or revelation of which in and manner to an unauthorized person is prohibited by law.

*This report concerns work done partly on behalf of the Division of Raw Materials of the U. S. Atomic Energy Commission. 


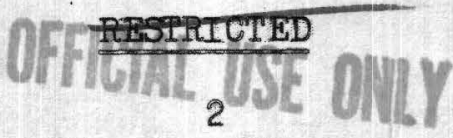

\author{
USGS - TEI -364 \\ GEOLOGY AND MINERALOGY
}

Distribution (Series A)

No. of copies

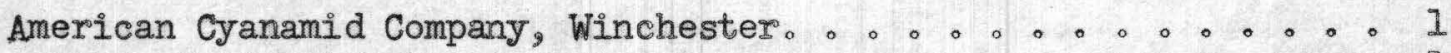

Argonne National Laboratory. . . . . . . . . . . . . . . . . . 1

Atomic Energy Commission, Washington ............... . 1

Battelle Memorial Institute, Columbus. . . . . . . . . . . . 1

Carbide and Carbon Chemicals Company, $Y-12$ Area. . . . . . . . I

Division of Raw Materials, Butte . . . . . . . . . . . . . . . 1

Division of Raw Materials, Denver. . . . . . . . . 。 。 . . . 1

Division of Raw Materials, Grants. . . . . .......... . 1

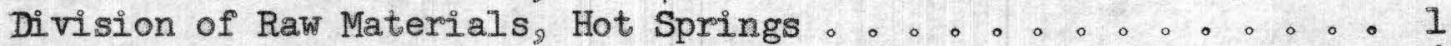

Division of Raw Materials, New York。 . o. . . . . 。 . . . . 6

Division of Raw Materials, Richfield . . . . . . . . . . . . . . 1

Division of Raw Materials, Salt Lake City。 . . 。 . . 。 . . . . . 1

Division of Raw Materials, Washington. . . . . . . . . . . . . 3

Dow Chemical Company, Pittsburg, . . . . . . . . . . . . . . . 1

Exploration Division, Grand Junction Operations Office 。 o . . 1

Grand Junction Operations Office . . . . . . . . . . . . . . . . 1

Technical Information Service, Oak Ridge o. . . . . . . . . . 6

Tennessee Valley Authority, Wilson Dam 。. 。 . . . 。 . . . 1

U. S. Geological Survey:

Alaskan Geology Branch Washington ............... 1

Fuels Branch, Washington 。. . . . . . . . . . . . . . . . . 1

Geochemistry and Petrology Branch, Washington, 。 。 。 。 。 . 1

Geophysics Branch, Washington. . . . . . . . . . . . . . . . 1

Mineral Deposits Branch, Washington。 . . . . . . . . . . . 2

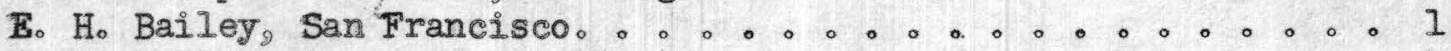

N. Mo Denson, Denver 。 . 。 。

C. E。 Dutton, Madison. . . . . . . . . . . . . . . . . . . . 01

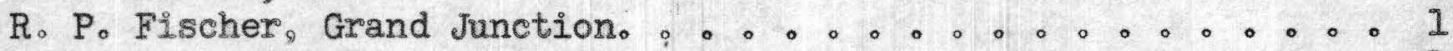

R. G. Petersen, Plant Citj .................... 1

M. R. Klepper, Washington, . . . . . . . . . . . . . . . . . . 1

A. H. Koschmann, Denver。 . . . . . . . . . . . . . . . . 1

R. A. Laurence, Knoxville. . . . . . . . . . . . . . . . . 1

D. M. Lemmon, Washington 。. . . . . . . . . . . . . . . 1

K. L. Buck, Denver 。. . . . . . . . . . . . . . . . . . . 2

R. Jo Roberts, Salt lake City。.................. . 1

R. W. Swanson, Spokane . . . . . . . . . . . . . . . . . . . . . 2

A. E. Weissenborn, Spokane . . . . . . . . . . . . . . . . . . 2

TEPCO, Washington:

Resource Compilation Section . . . . . . . . . . . . . . . . 2

Reports Processing Section . . . . . . . . . . . . . . . . 2 (Including master)

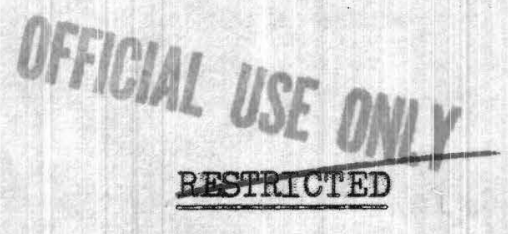


CONTENTS

Lot no. Page

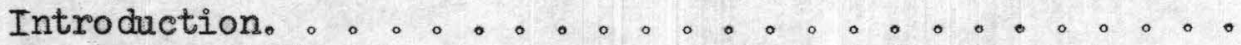

Acknowledgments。 ...................

Stratigraphy of the Phosphoria formation in southeastern Idaho

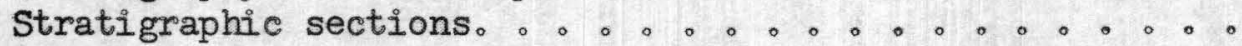

Literature cited. . . . . . . . . . . . . . . . . . . . .

Tables of stratigraphic sections, Idaho

Timber Creek .................. 1310

South Stewart Canyon ............... 1306

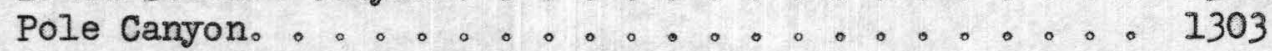

Spectrographic analyses ..............

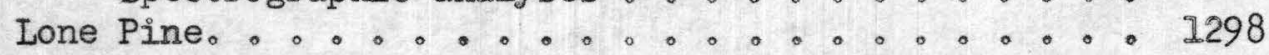

1298
1320

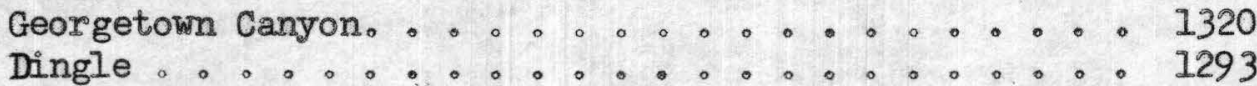

Hot Springs.

\section{ILLUSTRATIONS}

Figure 1. Outcrops of the Phosphoria formation in Idaho and localities sampled. ..............

2. Generalized section of Phosphoria formation at

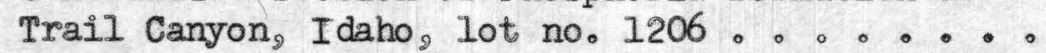




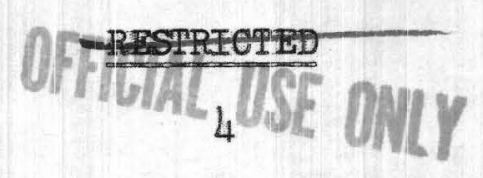

STRATIGRAPHIC SECTIONS OF THE PHOSPHORIA. FORMATION IN IDAHO, 1949, PART I

by R. P. Sheldon, M。 A. Warner, M。 E. Thompson, and H. Wo Peirce

\section{INTRODUCTION}

The U。 S. Geological Survey has recently measured and sampled the Phosphoria formation at many localities in Idaho and other western states. These data will not be fully synthesized and analyzed for several years, but segments of the data, accompanied by little or no interpretation, are published as preliminary reports as they are assembled. This report, which contains abstracts of many of the sections measured in southeastern Idaho (fig. 1), is one of this series and is the fourth report of data gathered in Idaho: it includes about half of the data gathered in Idaho in 1949. The field and laboratory procedures adopted in these investigations are described rather fully in a previous report (McKelrey and others, 1953a).

Many people have taken part in this investigation, which was organized and supervised by V. Eo McKelvey。 F。J.Anderson, D. F。 Davidson, R。 S。 Jones, R. A. Smart, R。 Go Waring, and Jo Do Weiser participated in the description of strata and the collection of sanples referred to in this report. $T$. $K$. Rigby assisted in the preparation of trenches and the collection, crushing, and splitting of samples in the field. The laboratory preparation of samples for chemical analysis was done in Denter, Colo., under the direction of W. P. Huleatto

The $\mathrm{P}_{2} \mathrm{O}_{5}$ and acid-insoluble analyses were made for the Survey by the U. S. Bureau of Mines at the Northwest Electrodevelopment Laboratory, Albany, Oreg., under the direction of $\mathrm{S}_{0} \mathrm{M}_{0}$ shelton and $\mathrm{M}_{0} \mathrm{~L}_{0}$ Wright。 The $\mathrm{Al}_{2} \mathrm{O}_{3}$ g 


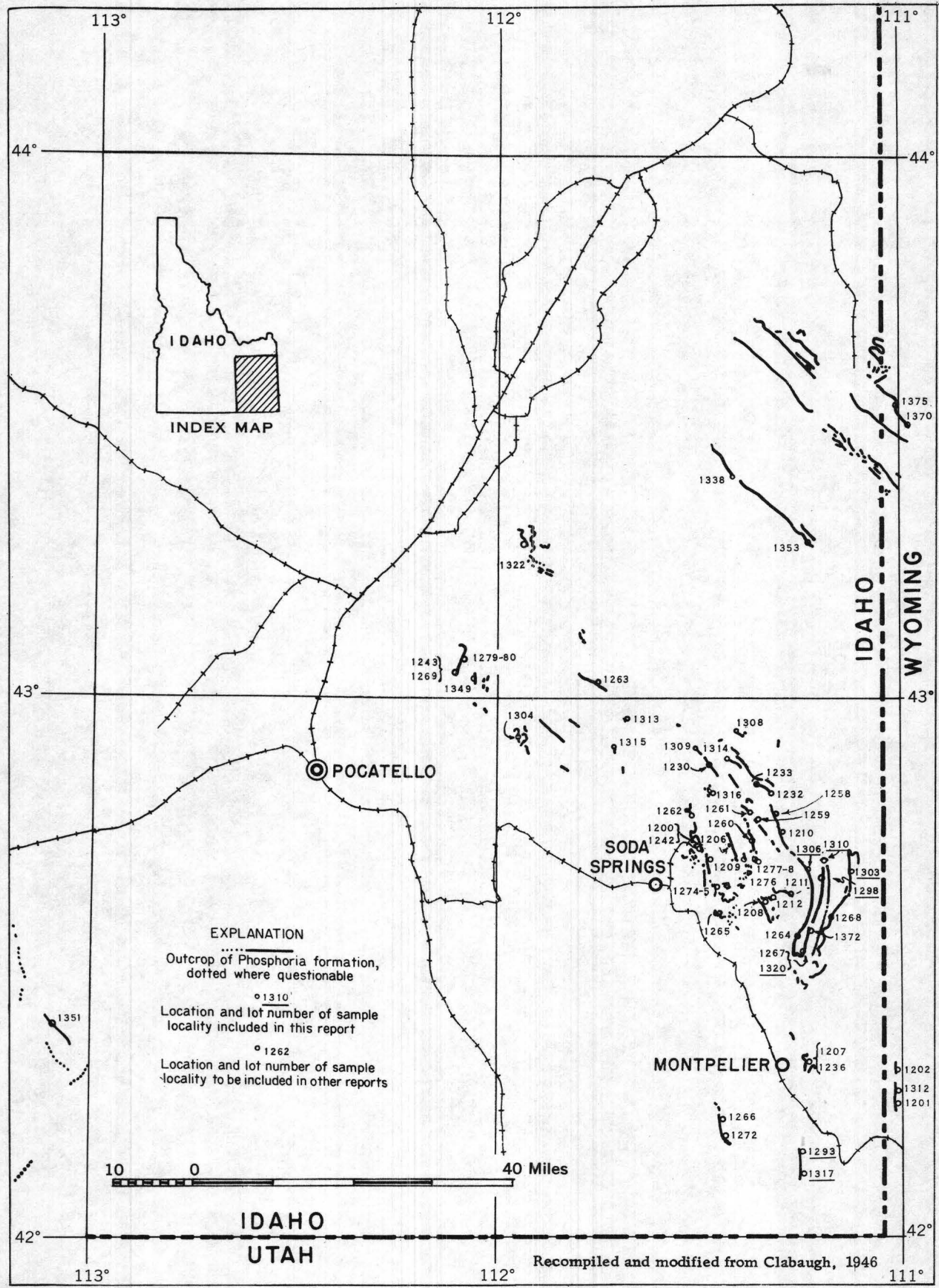

Figure 1. - Outcrops of the Phosphoria formation in Idaho and localities sampled. 


\section{OFFTHATISE ONIY}

$\mathrm{Fe}_{2} \mathrm{O}_{3}$, and loss-on-ignition analyses were made in the Trace Elements Section laboratory of the Survey in Washington, $D_{0} C_{0}$, under the direction of $J \circ C$. Rabbitt, by chemists H. Albexty, I. Barlow, A. Caemmerer, Fo J. Flanagan, F. S. Grimaldi, No Guttag, C. Hoy, Jo Jo Rowe, and W. P. Tucker, and the spectrographic analyses were made in this laboratory by $\mathrm{K}$. Valentine and C. I. Waring. The radioactivity analyses were made in the Trace Elements Section laboratory in Denver, Colo, , under the direction of L。 F。 Rader, by J. No Rosholt and in the Washington laboratory by F。 Jo Flanagan, B。 A. McCall, and $J_{0} J_{0}$ Warr, Jro The chemical uranium analyses were made in the Denver laboratory by G. T. Burrow and W. Mountjoy and in the Washington laboratory by A. B. Caemmerer, F。 Jo Flanagan, F. S. Grimaldi, N. Guttag, C. Hoy, A. Co Pietsch, and A. I. White.

The data were compiled largely by $K$. So Bergman under the supervision of R. W. Swanson. Organization of the tabular data was by Anita Wise.

\section{Acknowledgments}

Special thanks are due W. W. Rubey, Jo Steele Williams, and A. E. Weissenborn who have given much advice in planning and organizing the field program. The cost of both the field and laboratory investigations has been borne partly by the Division of Raw Materials of the Atomic Energy Commission. This support is gratefully acknowledged。

It is a pleasure to acknowledge the fine cooperation extended to the field parties by the local residents, property owners, and operating phosphate companies, who furnished information and services and gave access to property。 A. Jo Winters, Superintendent of the Montpelier Schools: E。 Mo Norris, CoT。 Russell, and L. E. Traeger of the Anaconda Copper Mining Company;

D. L。 King 


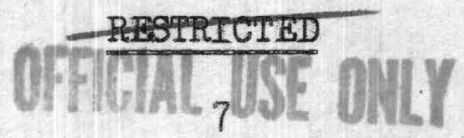

of the San Francisco Chemical Company; and G. A. McHugh and H. Bo Fowler of the Simplot Fertilizer Company have been especially helpful in this connection.

\section{STRATIGRAPHY OF THE PHOSPHORIA FORMATION IN SOUTHEASTERN IDAHO}

At its type locality in southeastern Idaho (Richards and Mansfield, 1912), the Phosphoria formation consists of a lower member, the phosphatic shale, about 180 feet thick and an upper member, the Rex chert, about 240 feet thick: another member, a thin-bedded cherty mudstone 15 to 75 feet thick, overlies the Rex chert in most of southeastern Idaho and western Wyoming, though it is not well defined at the type locality.

The Phosphoria formation overlies the Wells formation of Pennsylvanian age and underlies the Dinwoody formation of Triassic age. The upper 50 to 75 feet of the Wells formation consists of gray fossiliferous cherty limestone and contains some thin phosphatic layers. It may be the correlative of the lowermost member (A member) of the Phosphoria formation in Montana and the lower limestone member of the Park City formation in Utah (McKelvey, 1949).

In southeastern Idaho most of the phosphatic beds are in the phosphatic shale member, and it is on this member that most of our studies have been focused. It consists of many thin layers, some of which persist over the whole area. They may be grouped into several broad units, as yet unnamed, as shown in figure 2 .

\section{STRATIGRAPHIC SECTIONS}

Abstracts of stratigraphic sections measured at seven localities and the available analytical data are presented in the following pages. Their locations, as well as the locations of sections reported previously (McKelvey 


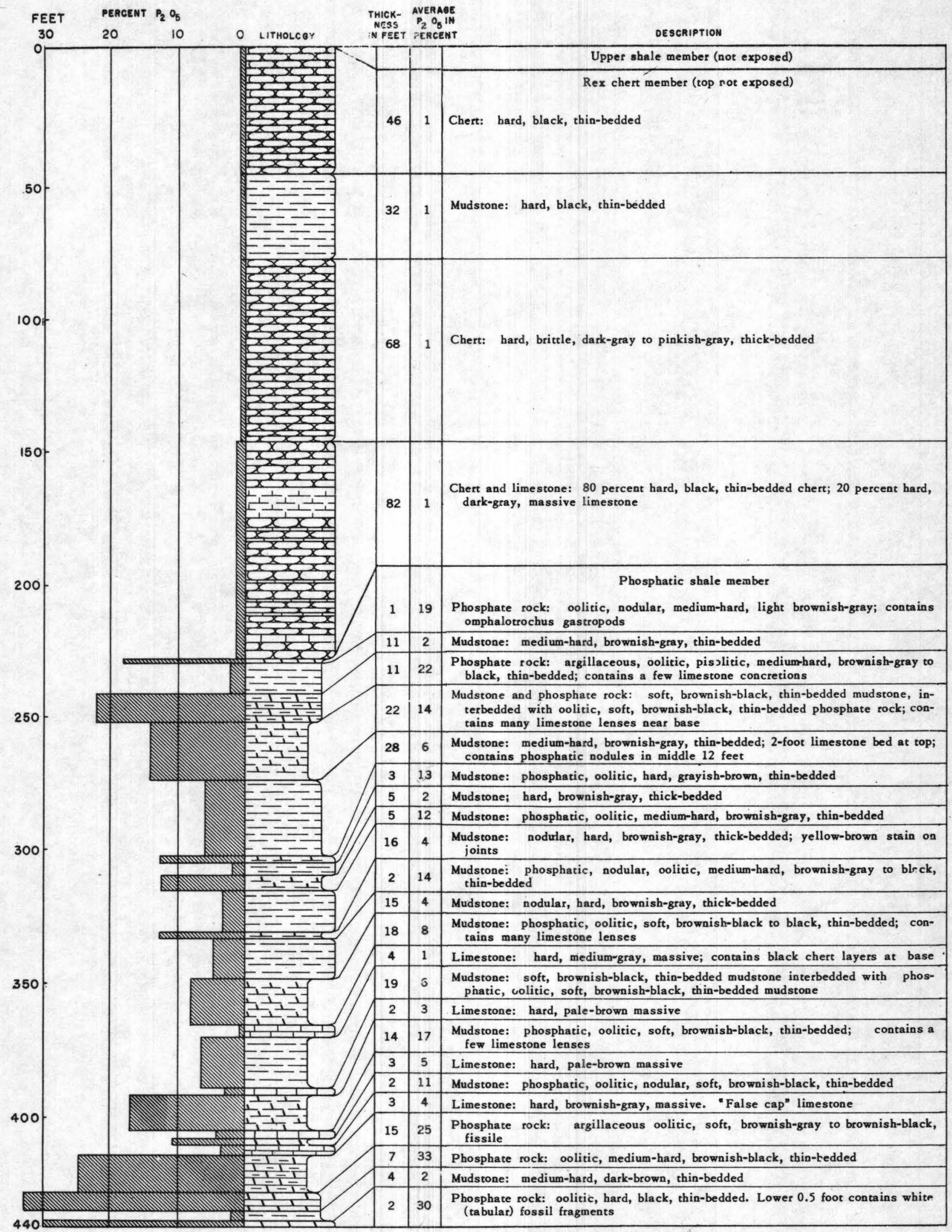

Wells formation

Figure 2. -Generalized section of the Phosphoria formation at Trail Canyon (lot no. 1206). 


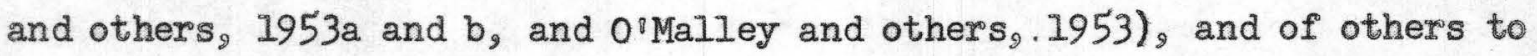
be reported later, are shown in figure 1.

The semiquantitative spectrographic analyses are based upon comparisons with a standard plate representing known quantities of the elements tested for and made at the same exposure. Greater sensitivities for many elements can be obtained by additional exposures. The standard sensitivities for the elements noted in this report are as follows:

\section{Spectrographie Sensitivities}

\begin{tabular}{|c|c|c|c|c|c|c|c|c|c|c|c|}
\hline Al & $\ldots$ & 0.0001 & $\mathrm{Gd}$ & $\ldots$ & .01 & $\mathrm{Nd}$ & $\ldots$ & .01 & $\mathrm{Na}$ & $\ldots$ & $* 01$ \\
\hline $\mathrm{Sb}$ & $\ldots$ & .01 & $\mathrm{Ga}$ & $\ldots$ & .01 & Ni & $\ldots$ & .01 & Sr & $\ldots$ & .01 \\
\hline As & $\ldots$ & .1 & Ge & $\ldots$ & .001 & $\mathrm{Nb}$ & $\ldots$ & .01 & $\mathrm{Ta}$ & $\ldots$ & .1 \\
\hline $\mathrm{Ba}$ & $\ldots$ & .0001 & $\mathrm{Au}$ & $\ldots$ & .01 & os & $\ldots$ & $\circ$ & $\mathrm{Te}$ & $\ldots$ & .1 \\
\hline $\mathrm{Be}$ & $\ldots$ & .0001 & $\mathrm{Hf}$ & $\ldots$ & .1 & $\mathrm{Pd}$ & $\ldots$ & .01 & $\mathrm{~Tb}$ & $\ldots$ & .01 \\
\hline Bi & $\ldots$ & .001 & Ho & $\ldots$ & .01 & $P$ & $\ldots$ & .2 & $\mathrm{TI}$ & $\ldots$ & .1 \\
\hline B & $\ldots$ & .001 & In & $\ldots$ & .001 & Pt & $\ldots$ & .01 & $\mathrm{Th}$ & $\ldots$ & .1 \\
\hline $\mathrm{Cd}$ & $\ldots$ & .01 & Ir & $\ldots$ & .1 & $\mathrm{~K}$ & $\ldots$ & $* 1.0$ & $\mathrm{Tm}$ & $\ldots$ & .01 \\
\hline $\mathrm{Ca}$ & $\ldots$ & .001 & $\mathrm{Fe}$ & $\ldots$ & .001 & Pr & $\ldots$ & .01 & $\mathrm{Sn}$ & $\ldots$ & .01 \\
\hline $\mathrm{Ce}$ & $\ldots$ & .1 & $\mathrm{La}$ & $\ldots$ & 0.01 & $\mathrm{Re}$ & $\ldots$ & .1 & $\mathrm{TI}$ & $\ldots$ & .001 \\
\hline Cs & $\ldots$ & $* 1.0$ & $\mathrm{~Pb}$ & $\ldots$ & .01 & $\mathrm{Rh}$ & $\ldots$ & .01 & W & $\ldots$ & .1 \\
\hline Cr & $\ldots$ & .001 & Li & $\ldots$ & $* 1$ & $\mathrm{Rb}$ & $\ldots$ & 10.0 & $\nabla$ & $\cdots$ & .01 \\
\hline Co & $\ldots$ & .01 & Lu. & $\ldots$ & .01 & $\mathrm{Ru}$ & $\ldots$ & .01 & $\mathrm{Yb}$ & $\ldots$ & .0001 \\
\hline $\mathrm{Cu}$ & $\ldots$ & .0001 & $\mathrm{Mg}$ & $\ldots$ & .0001 & $\mathrm{Sm}$ & & .1 & $Y$ & $\ldots$ & .001 \\
\hline Dy & $\ldots$ & .01 & $\mathrm{Mn}$ & $\ldots$ & .001 & Sc & & .001 & $\mathrm{zn}$ & $\ldots$ & .01 \\
\hline Er & $\cdots$ & .01 & $\mathrm{Hg}$ & $\cdots$ & .1 & Si & $\ldots$ & .0001 & $2 r$ & $\ldots$ & .001 \\
\hline Eu & $\ldots$ & .01 & Mo & $\ldots$ & .001 & $\mathrm{Ag}$ & & .0001 & & & \\
\hline
\end{tabular}

*A greater sensitivity may be obtained by additional exposures. 
Clabaugh, P. S.g 1946, Permian phosphate deposits of Montana, Idaho, Wyoming, and Utah: U. S. Geol. Survey, Strategic Minerals Inv. Prelim. Map $3-198$.

McKelvey, Vo. E., 1949, Geological studies of the western phosphate field: Am. Inst. Min. Met. Eng. Mining Trans., v。 184, p. 270-279.

McKelrey, Vo Eo, Davidson, Do F, O Malley, Fo Wog and Smith, L. Eog 1953a, Stratigraphic sections of the Phosphoria formation in Idaho, $1947-48$, Part I: U. S. Geol. Survey Circ. 208.

McKelvey, Vo E., Armstrong, F。 C., Campbell, R. M., and Gulbrandsen, R. A, g $1953 \mathrm{~b}$, Stratigraphic sections of the Phosphoria formation in Idaho, 1947-48, Part II: U. S. Geol. Survey Cire. 30L.

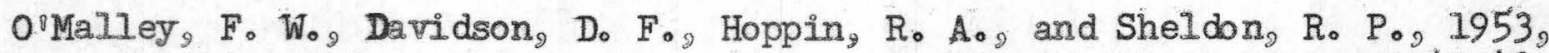
Stratigraphic sections of the Phosphoria formation in Idaho, $1947-48$, Part III: U. S. Geol. Survey Circ. 262.

Richards, R。 Wo, and Mansfield, Go Ro, 1912, The Bannock overthrust: a major fault in southeastern Idaho and northeastern Utah: Jour. Geology, vo 20, p. 684 。 
Timber Creek, Idaho, lot 1310

Phosphatic shale member of the Phosphoria formation sampled in bulldozer trench on east limb of Snowdrift anticline, $\mathrm{S} \frac{1}{2} \mathrm{sec}$. $21, \mathrm{~T} .8 \mathrm{~S}$., R. $45 \mathrm{E}$., Caribou County, Idaho. Section measured by M. A. Warner, R. G. Waring, R. A. Smart, J. D. Weiser, and M. E. Thompson and sampled by
Smart, Waring, H. W. Peirce, and Warner in August 1949. Samples analyzed for $\mathrm{P}_{2} \mathrm{O}_{5}$ and acid insoluble by U. S. Bureau of Mines laboratory, Smart, Waring, H. W. Peirce, and Warner in August 1949. Samples analyzed for $\mathrm{P}_{2} \mathrm{O}_{5}$ and acid insoluble by U. S. Bureau of $\mathrm{M}$
Albany, Oreg., and for other constituents by Trace Elements Section laboratory, U. S. Geological Survey, Washington, D. C.

Samples analyzed for $\mathrm{eU}$ and chem. $U$ by the U. S. Geological Survey laboratory, Geochemistry and Petrology Branch.

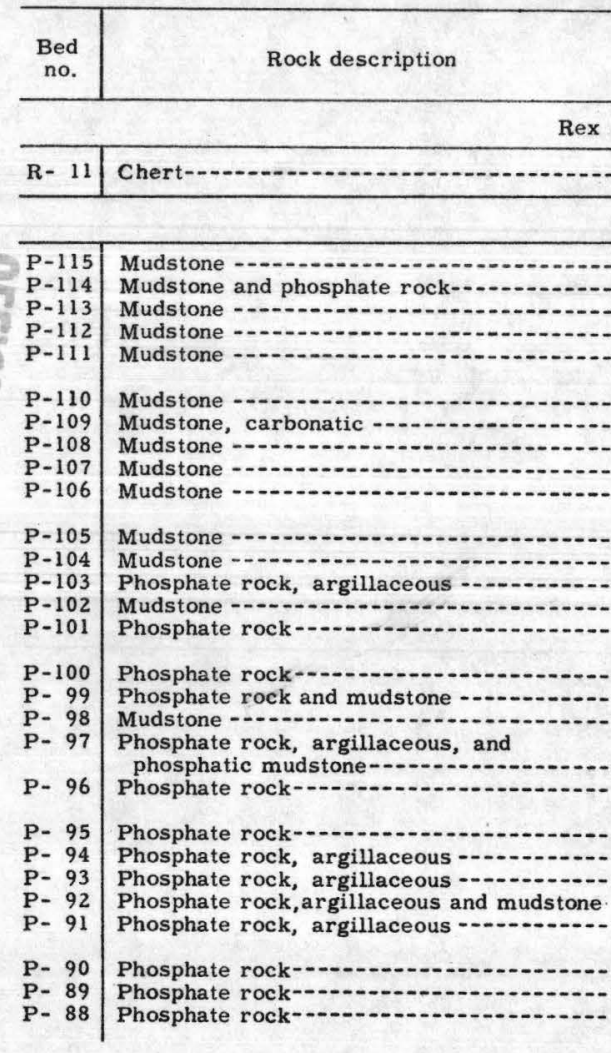

Lot no. 1310

\begin{tabular}{|c|c|c|c|c|c|c|c|}
\hline \multirow{2}{*}{$\begin{array}{c}\text { Sample } \\
\text { no. }\end{array}$} & \multirow{2}{*}{$\begin{array}{c}\text { Thickness } \\
\text { (feet) }\end{array}$} & \multicolumn{5}{|c|}{ Chemical analyses (percent) } & \multirow{2}{*}{$\begin{array}{l}\text { Cumulative } \\
\text { thickness } \\
\text { (feet) }\end{array}$} \\
\hline & & & $\mathrm{Al}_{2} \mathrm{O}_{3}$ & $\mathrm{Fe}_{2} \mathrm{C}$ & & $\begin{array}{r}A \\
\text { ins }\end{array}$ & \\
\hline
\end{tabular}

\begin{tabular}{l|l} 
Thickness $x$ \\
percent $\mathrm{P}_{2} \mathrm{O}_{5}$ \\
(cumulative)
\end{tabular}
(percent)
(pium content Chem. U

Thickness $x$ percent eU
(cumulative)

ex chert member of Phosphoria formation-basal bed only

\begin{tabular}{c|c|c|c|c|c|}
\hline Phosphatic shale member of Phosphoria formation &
\end{tabular}

\begin{tabular}{l|l|l|l|l|l}
\hline 94.0 & 2.2 & -- & 0.000 & 0.001 & - \\
\hline
\end{tabular}

Poria formation

\begin{tabular}{|c|}
\hline $\begin{array}{l}\text { 4234-MAW } \\
4233-\mathrm{MAW} \\
4232-\mathrm{MAW} \\
4031-\mathrm{MAW} \\
4030-\mathrm{MAW}\end{array}$ \\
\hline $\begin{array}{l}\text { 4029-MAW } \\
\text { 4228-MAW } \\
\text { 4227-MAW } \\
4226-\mathrm{MAW} \\
\text { 4459-RGW }\end{array}$ \\
\hline $\begin{array}{l}\text { 4458- RGW } \\
\text { 4457- RGW } \\
\text { 4456- RGW } \\
\text { 4455- RGW } \\
\text { 4454- RGW }\end{array}$ \\
\hline $\begin{array}{l}\text { 4453-RGW } \\
4452-\mathrm{RGW} \\
4451-\mathrm{RGW}\end{array}$ \\
\hline $\begin{array}{l}\text { 4450-RGW } \\
5209-\text { RGW }\end{array}$ \\
\hline $\begin{array}{l}\text { 5208- RGW } \\
5207-\text { RGW } \\
5206-\text { RGW } \\
5205-\text { RGW } \\
4445-\text { RAS }\end{array}$ \\
\hline $\begin{array}{l}\text { 4444- RAS } \\
4443-\text { RAS } \\
4442-\text { RAS }\end{array}$ \\
\hline
\end{tabular}

\begin{tabular}{l|}
1.5 \\
1.2 \\
2.7 \\
1.5 \\
1.8 \\
2.5 \\
2.7 \\
1.3 \\
2.0 \\
.9 \\
.6 \\
3.3 \\
.7 \\
3.0 \\
1.7 \\
1.3 \\
1.2 \\
1.0 \\
1.0 \\
1.0 \\
1.9 \\
.7 \\
.6 \\
1.8 \\
1.1 \\
.9 \\
2.2
\end{tabular}

\begin{tabular}{|c|c|c|c|}
\hline 1.7 & -- & -. & -- \\
\hline 10.4 & $\cdots$ & $-\cdot$ & . \\
\hline 4. 7 & -- & - & -- \\
\hline $\begin{array}{l}1.8 \\
1.8\end{array}$ &.- &.- & - \\
\hline 2. 0 & -- & -- & -- \\
\hline & -- & $\because$ & -- \\
\hline 1.6 & - & -- & -- \\
\hline 1.1 & -- & -- & -- \\
\hline 3. 0 & -. & -. & -- \\
\hline 3. 0 & $\div-$ & $\cdots$ & -- \\
\hline $\begin{array}{r}28.2 \\
3.8\end{array}$ & -- & $-\overline{-}$ & -- \\
\hline 35.7 & 0.86 & 0.35 & 4.75 \\
\hline 36.0 & 85 & .33 & 5.55 \\
\hline $\begin{array}{r}29.6 \\
6.2\end{array}$ & $\begin{array}{l}2.98 \\
9.05\end{array}$ & $\begin{array}{l}1.05 \\
2.78\end{array}$ & $\begin{array}{l}4.50 \\
5.65\end{array}$ \\
\hline 21.1 & 6.98 & 2. 10 & 4.80 \\
\hline 34.4 & 1.34 & 60 & 2.75 \\
\hline 32.5 & 1.84 & .80 & 2.80 \\
\hline & 3.90 & 1.13 & 3. 70 \\
\hline 24.1 & 4.06 & 1.38 & 4.90 \\
\hline 15. & 6.08 & 1.98 & 6.93 \\
\hline & 5.68 & 2.00 & 5.50 \\
\hline 30.8 & 2. 46 & .75 & 3.45 \\
\hline $\begin{array}{l}37.1 \\
35.5\end{array}$ & $\begin{array}{l}1.34 \\
1.18\end{array}$ & .33 & 3.15 \\
\hline & 1.18 & .35 & 4.88 \\
\hline
\end{tabular}

\begin{tabular}{r|}
91.0 \\
60.6 \\
71.5 \\
65.7 \\
78.6 \\
79.1 \\
68.8 \\
82.9 \\
78.0 \\
80.5 \\
65.6 \\
73.6 \\
20.2 \\
71.9 \\
3.4 \\
3.2 \\
17.5 \\
71.3 \\
36.7 \\
8.2 \\
12.7 \\
28.5 \\
30.6 \\
46.9 \\
43.0 \\
14.5 \\
3.6 \\
4.0
\end{tabular}

1.5
2.7
5.4
6.9
8.7
11.2
13.9
15.2
17.2
18.1
18.7
22.0
22.7
25.7
27.4
28.7
29.9
31.5
32.5
33.5
35.4
36.1
36.7
37.5
38.6
39.7
40.6
42.8

2.55
15.03
27.72
35.37
38.61
43.61
45.23
46.01
49.21
50.20
52.00
61.90
81.64
93.04
153.73
200.53
236.05
245.97
267.07
301.47
363.22
380.79
395.25
407.57
428.03
461.91
495.30
573.40

0.001
.005
.004
.005
.003
.003
.001
.004
.002
.002
.004
.004
.009
.004
.009
.011
.009
.004
.007
.013
.013
.010
.008
.007
.006
.014
.016
.017

0.003
.008
.004
.006
.002
.002
.001
.001
.002
.002
.003
.004
.011
.004
.012
.014
.010
.003
.008
.015
.016
.012
.008
.008
.007
.018
.019
.019

0. 004

.014
.025

.025
.034
.038

.038

.042
.045
.046

.046

.051

.054

.064
.067
.075

.087
.087

.126
.138

.138
.142

.150
.165

.196

204
.209

.215
.223

.243
.260
.302
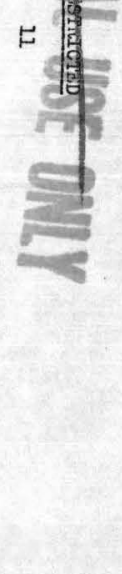

V - Idaho - 1 
Timber Creek-Continued

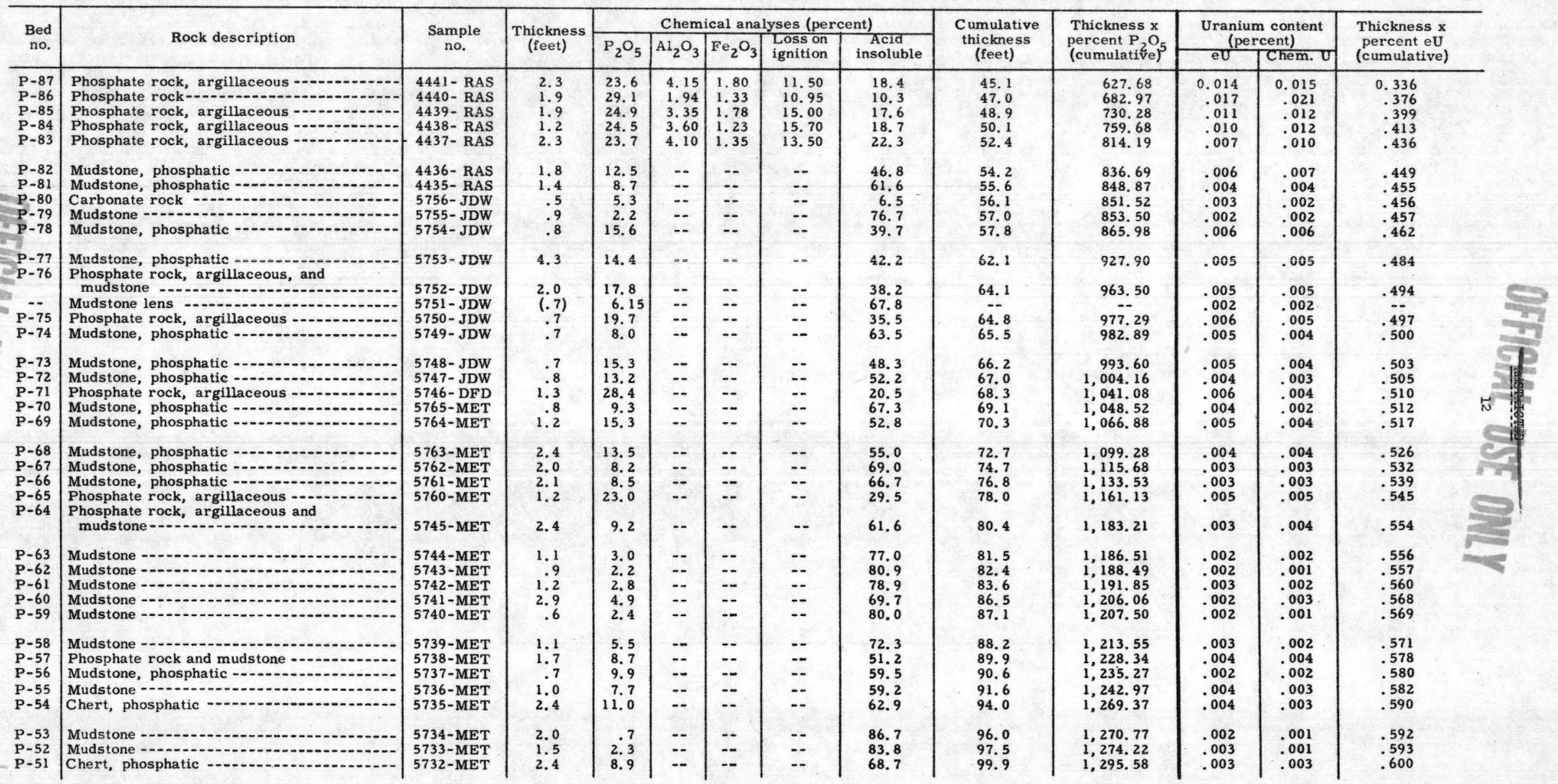




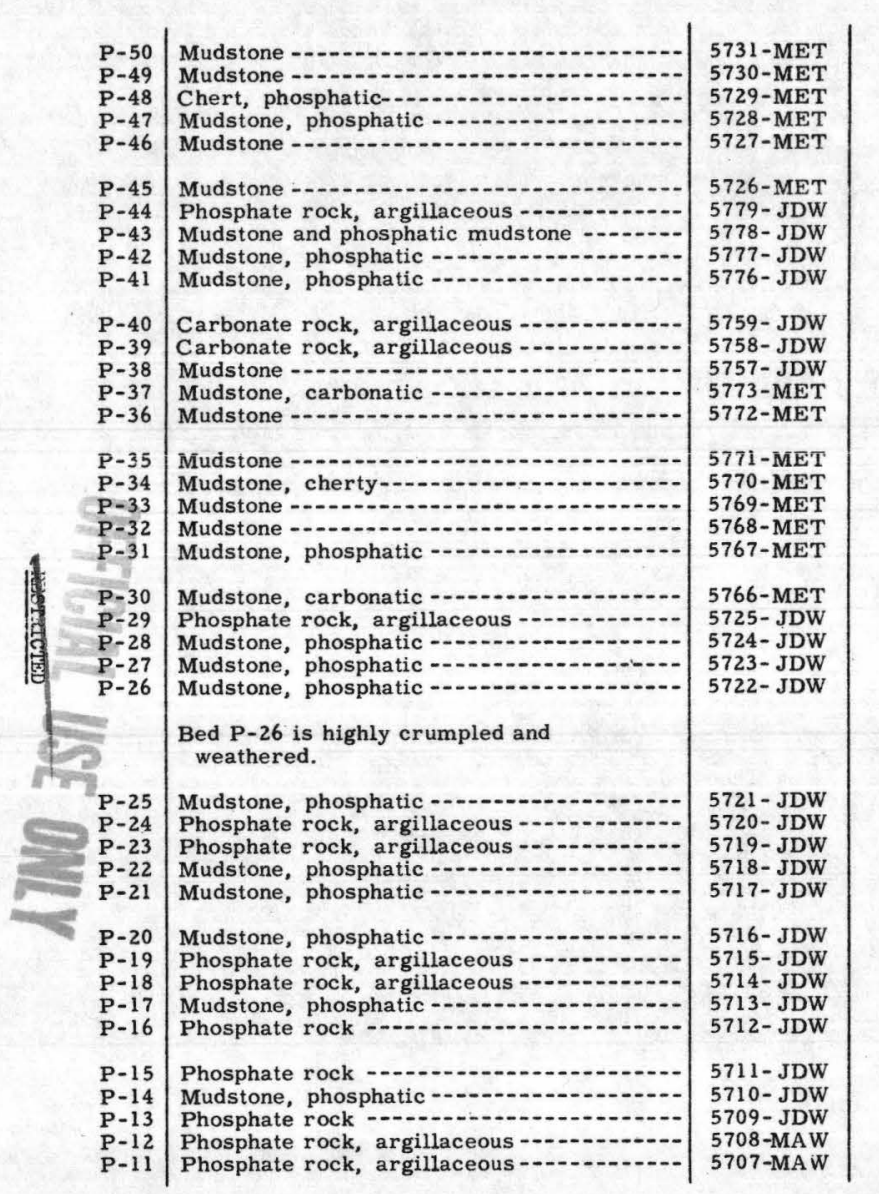

\begin{tabular}{|c|c|c|c|c|}
\hline 3.3 & .7 & - & -- & -- \\
\hline $\begin{array}{l}1.2 \\
4.9\end{array}$ & $\begin{array}{r}1.0 \\
15.5\end{array}$ & - & $\therefore$ & 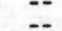 \\
\hline .9 & 9.7 & -. & -- & -- \\
\hline 2. 1 & 6.1 & -- & -- & - \\
\hline 2.4 & 6.4 & -- & -- & - \\
\hline .7 & 23.2 & -- & -- & $=$ \\
\hline & $\begin{array}{l}10.4 \\
14.1\end{array}$ & -- & $\bar{z}$ & $=$ \\
\hline $\begin{array}{l}4.6 \\
1.9\end{array}$ & $\begin{array}{r}8.4 \\
\end{array}$ & -. & - & - \\
\hline 1.7 & 1.0 & -- & -- & - \\
\hline 1.2 & 1.1 & -- & - & -- \\
\hline 1.2 & 2.5 & -- & -- & $\cdots$ \\
\hline 1.3 & 2.7 & -. & -- & -- \\
\hline .5 & 2.0 & -- & -- & -- \\
\hline .9 & 2. 0 & $=$ & -- & -- \\
\hline 0 & 3.5 & -- & $\overline{-}$ & $=$ \\
\hline $\begin{array}{l}4.0 \\
3.2\end{array}$ & 8.7 & - & - & - \\
\hline .7 & 5. 7 & -- & -- & -- \\
\hline 5 & 18.2 & -- & -- & 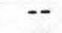 \\
\hline 1.3 & 10.4 & $\because$ & $\overline{-}$ & $=$ \\
\hline 3.2 & 14.2 & -. & -. & -. \\
\hline 1.5 & 15.4 & -- & - & - \\
\hline 7 & $\begin{array}{r}25.3 \\
25.4\end{array}$ & - & $\overline{-}$ & $\because$ \\
\hline 3.0 & $\begin{array}{l}15.8 \\
\end{array}$ & -. & - & - \\
\hline 3.4 & 12.7 & -- & -- & -- \\
\hline 1.5 & 8.8 & -- & -- & -- \\
\hline & 25.4 & -- & -- & -- \\
\hline $\begin{array}{l}2.1 \\
1.3\end{array}$ & $\begin{array}{r}21.0 \\
8.4\end{array}$ & -- & $=$ & $=$ \\
\hline 5.0 & 31.5 & 1.53 & .70 & 7.30 \\
\hline 1.4 & 29.4 & 2.04 & .85 & 7.15 \\
\hline & 16.3 & 6.20 & 2.05 & 9.35 \\
\hline & $\begin{array}{r}30.4 \\
15.9\end{array}$ & $\begin{array}{l}2.30 \\
7.32\end{array}$ & $\begin{array}{r}.95 \\
95\end{array}$ & 9.00 \\
\hline 04 & 27.4 & 3.30 & 1.18 & 6.75 \\
\hline
\end{tabular}

\begin{tabular}{l|}
86.2 \\
86.6 \\
50.1 \\
59.6 \\
68.8 \\
65.6 \\
15.2 \\
51.5 \\
36.6 \\
60.7 \\
29.1 \\
43.6 \\
78.0 \\
54.3 \\
64.3 \\
74.3 \\
85.3 \\
72.3 \\
66.7 \\
57.7 \\
52.7 \\
31.9 \\
56.1 \\
42.2 \\
40.1 \\
\\
\\
45.4 \\
17.6 \\
22.5 \\
47.5 \\
51.3 \\
61.5 \\
19.3 \\
31.9 \\
64.7 \\
8.9 \\
14.9 \\
40.5 \\
11.5 \\
38.7 \\
16.2 \\
\end{tabular}

103.2
104.4
109.3
110.2
112.3
114.7
116.4
118.3
122.5
124.4
126.1
127.3
128.5
130.8
132.1
133.6
134.5
136.5
141.1
144.3
145.0
148.5
149.8
152.5
155.7

153
157.2
158.9
160.1
163.1
166.5
168.0
171.0
173.1
174.4
179.4
180.8
181.8
182.7
183.4
185.8

$1,297.89$
$1,299.09$
$1,375.04$
$1,383.77$
$1,396.58$
$1,411.94$
$1,451.38$
$1,471.14$
$1,530.36$
$1,546.32$
$1,548.02$
$1,549.34$
$1,552.34$
$1,555.10$
$1,558.61$
$1,561.61$
$1,563.41$
$1,570.41$
$1,593.41$
$1,621.25$
$1,625.24$
$1,688.94$
$1,702.46$
$1,742.42$
$1,787.86$

$1,$.
$1,810.96$
$1,853.97$
$1,984.45$
$1,975.03$
$1,988.23$
$2,064.43$
$2,108.53$
$2,119.45$
$2,276.95$
$2,318.11$
$2,334.41$
$2,361.77$
$2,372.90$
$2,438.66$

\begin{tabular}{|c|c|}
\hline $\begin{array}{l}.001 \\
.003 \\
.004 \\
.003 \\
.003\end{array}$ & $\begin{array}{l}.001 \\
.001 \\
.003 \\
.003 \\
.002\end{array}$ \\
\hline $\begin{array}{l}.004 \\
.014 \\
.006 \\
.005 \\
.003\end{array}$ & $\begin{array}{l}.003 \\
.016 \\
.005 \\
.005 \\
.002\end{array}$ \\
\hline $\begin{array}{l}.003 \\
.002 \\
.003 \\
.002 \\
.003\end{array}$ & $\begin{array}{l}.001 \\
.001 \\
.002 \\
.001 \\
.002\end{array}$ \\
\hline $\begin{array}{l}.002 \\
.002 \\
.004 \\
.003 \\
.003\end{array}$ & $\begin{array}{l}.002 \\
.002 \\
.003 \\
.002 \\
.002\end{array}$ \\
\hline $\begin{array}{l}.004 \\
.004 \\
.004 \\
.003 \\
.005\end{array}$ & $\begin{array}{l}.003 \\
.004 \\
.002 \\
.003 \\
.003\end{array}$ \\
\hline $\begin{array}{l}.007 \\
.005 \\
.010 \\
.005 \\
.006\end{array}$ & $\begin{array}{l}.003 \\
.005 \\
.009 \\
.004 \\
.004\end{array}$ \\
\hline $\begin{array}{r}.005 \\
.011 \\
.010 \\
.006 \\
.012\end{array}$ & $\begin{array}{l}.002 \\
.009 \\
.009 \\
.003 \\
.012\end{array}$ \\
\hline $\begin{array}{l}.014 \\
.008 \\
.012 \\
.009 \\
.007\end{array}$ & $\begin{array}{l}.013 \\
.006 \\
.010 \\
.005 \\
.007\end{array}$ \\
\hline
\end{tabular}


Timber Creek-Continued

\begin{tabular}{|c|c|c|c|c|c|c|c|c|c|c|c|c|c|}
\hline \multirow{2}{*}{$\begin{array}{l}\text { Bed } \\
\text { no. }\end{array}$} & \multirow[b]{2}{*}{ Rock description } & \multirow{2}{*}{$\begin{array}{c}\text { Sample } \\
\text { no. }\end{array}$} & \multirow{2}{*}{$\begin{array}{c}\text { Thickness } \\
\text { (feet) }\end{array}$} & \multicolumn{5}{|c|}{ Chemical analyses (percent) } & \multirow{2}{*}{$\begin{array}{l}\text { Cumulative } \\
\text { thickness } \\
\text { (feet) }\end{array}$} & \multirow{2}{*}{$\begin{array}{l}\text { Thickness } x \\
\text { percent } \mathrm{P}_{2} \mathrm{O}_{5} \\
(\text { cumulative) }\end{array}$} & \multirow{2}{*}{\multicolumn{2}{|c|}{$\begin{array}{c}\text { Uranium content } \\
\text { (percent) }\end{array}$}} & \multirow{2}{*}{$\begin{array}{l}\text { Thickness } \mathbf{x} \\
\text { percent eU } \\
\text { (cumulative) }\end{array}$} \\
\hline & & & & $\mathrm{P}_{2} \mathrm{O}_{5}$ & $\mathrm{Al}_{2} \mathrm{O}_{3}$ & $\mathrm{Fe}_{2} \mathrm{O}_{3}$ & $\begin{array}{l}\text { Loss on } \\
\text { ignition }\end{array}$ & $\begin{array}{c}\text { Acid } \\
\text { insoluble }\end{array}$ & & & & & \\
\hline $\begin{array}{l}\text { P-10 } \\
\text { P- } 9 \\
\text { P- } 8 \\
\text { P- } 7 \\
\text { P- } 6\end{array}$ & $\begin{array}{l}\text { Phosphate rock, argillaceous } \\
\text { Phosphate rock, argillaceous } \\
\text { Phosphate rock } \\
\text { Phosphate rock- } \\
\text { Phosphate rock }\end{array}$ & $\begin{array}{l}\text { 5706-MAW } \\
\text { 5705-MAW } \\
\text { 5704-MAW } \\
\text { 5239-MAW } \\
\text { 5703-MAW }\end{array}$ & $\begin{array}{l}1.2 \\
2.1 \\
1.9 \\
2.9 \\
1.2\end{array}$ & $\begin{array}{l}25.0 \\
22.5 \\
32.1 \\
33.1 \\
31.6\end{array}$ & $\begin{array}{l}2.76 \\
4.65 \\
1.47 \\
.70 \\
1.17\end{array}$ & $\begin{array}{l}1.48 \\
2.00 \\
2.88 \\
.53 \\
.68\end{array}$ & $\begin{array}{l}5.67 \\
7.50 \\
6.40 \\
7.55 \\
6.20\end{array}$ & $\begin{array}{r}24.8 \\
24.3 \\
3.5 \\
2.3 \\
6.0\end{array}$ & $\begin{array}{l}187.0 \\
189.1 \\
191.0 \\
193.9 \\
195.1\end{array}$ & $\begin{array}{l}2,468.66 \\
2,515.91 \\
2,576.90 \\
2,672.89 \\
2,710.81\end{array}$ & $\begin{array}{l}.003 \\
.004 \\
.009 \\
.024 \\
.012\end{array}$ & $\begin{array}{l}.003 \\
.003 \\
.008 \\
.025 \\
.013\end{array}$ & $\begin{array}{r}.988 \\
.994 \\
1.009 \\
1.082 \\
1.097\end{array}$ \\
\hline $\begin{array}{l}\text { P- } 5 \\
\text { P- } 4 \\
\text { P- } 3 \\
\text { P- } 2 \\
\text { P- } 1\end{array}$ & $\begin{array}{l}\text { Mudstone } \\
\text { Mudstone } \\
\text { Mudstone } \\
\text { Mudstone - } \\
\text { Phosphate rock, argillaceous }\end{array}$ & $\begin{array}{l}\text { 5702-MAW } \\
5701-\mathrm{MAW} \\
\text { 4238-MAW } \\
4237-\mathrm{MAW} \\
\text { 4236-MAW }\end{array}$ & $\begin{array}{l}1.2 \\
2.4 \\
1.2 \\
.7 \\
.5\end{array}$ & $\begin{array}{r}.3 \\
.4 \\
.7 \\
7.6 \\
26.4\end{array}$ & $\begin{array}{l}- \\
=- \\
z \\
--\end{array}$ & $\begin{array}{l}\because \\
\because \\
\because- \\
-\end{array}$ & $\begin{array}{l}- \\
\because \\
\because- \\
-\end{array}$ & $\begin{array}{l}75.3 \\
71.9 \\
70.3 \\
57.0 \\
15.5\end{array}$ & $\begin{array}{l}196.3 \\
198.7 \\
199.9 \\
200.6 \\
201.1\end{array}$ & $\begin{array}{l}2,711.17 \\
2,712.13 \\
2,712.97 \\
2,718.29 \\
2,731.49\end{array}$ & $\begin{array}{l}.004 \\
.001 \\
.002 \\
.004 \\
.009\end{array}$ & $\begin{array}{l}.001 \\
.001 \\
.001 \\
.002 \\
.009\end{array}$ & $\begin{array}{l}1.098 \\
1.101 \\
1.102 \\
1.103 \\
1.108\end{array}$ \\
\hline
\end{tabular}


South Stewart Canyon, Idaho, lot 1306

Phosphatic shale member of Phosphoria formation sampled in two hand trenches on overturned east limb of the Dry Valley anticline in South Stewart Canyon, SE $\frac{1}{4} \mathrm{SE} \frac{1}{4}$ sec. 31, T. 8 S., R. 45 E., Caribou County, Idaho. Because of limitations of hand trenches and complicated local structure, the total thickness of the phosphatic shale member is probably in error. Section measured by R. P. Sheldon, R. G. Waring, M. A. Warner, D. F. Davidson, M. E. Thompson, J. D. Weiser, and H. W. Peirce and sampled by Waring, Warner, Peirce, and R. A. Smart in August 1949 .

Samples analyzed for $\mathrm{eU}$ and chem. U by the U. S. Geological Survey laboratory, Geochemistry and Petrology Branch. Section laboratory, U. S. Geological Survey, Washington, D. C.

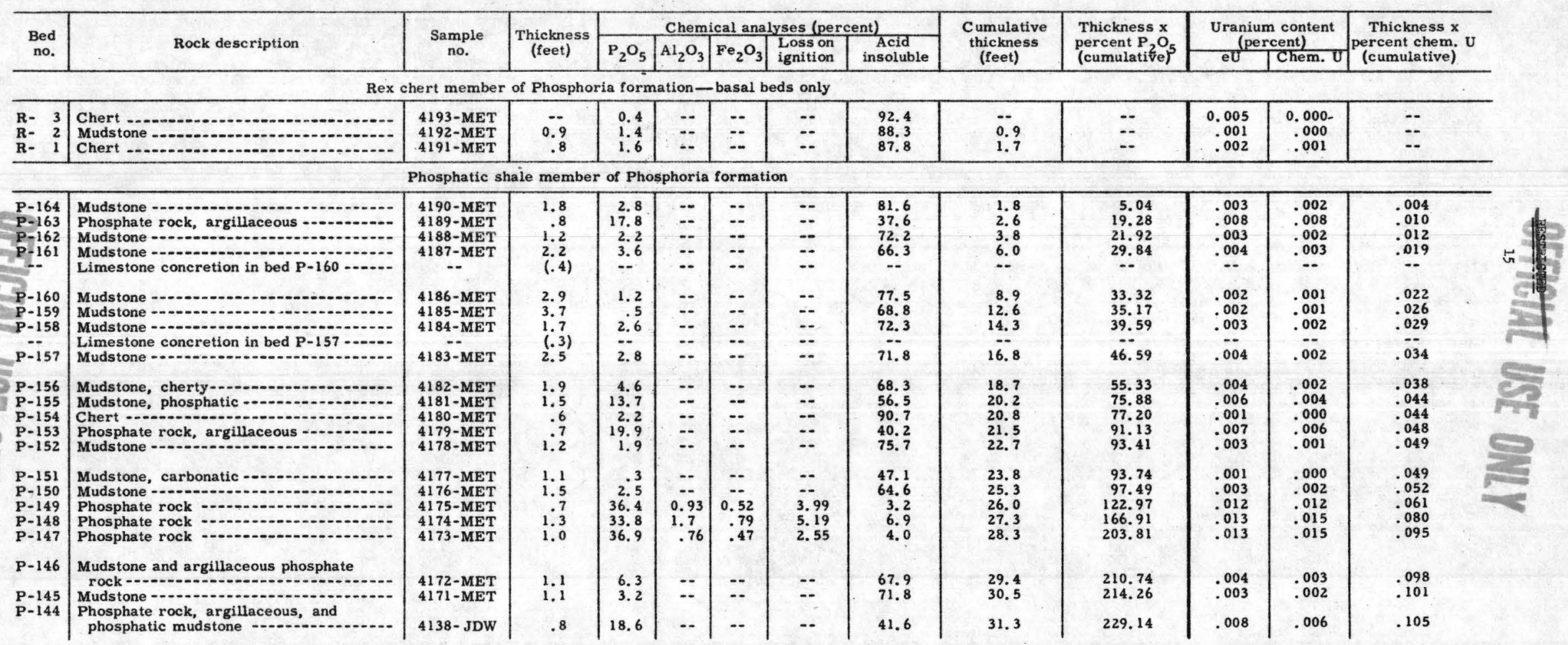


South Stewart Canyon-Continued

\begin{tabular}{|c|c|c|c|c|c|c|c|c|c|c|c|c|c|}
\hline \multirow{2}{*}{$\begin{array}{r}\text { Bed } \\
\text { no. }\end{array}$} & \multirow{2}{*}{ Rock description } & \multirow{2}{*}{$\begin{array}{c}\text { Sample } \\
\text { no. }\end{array}$} & \multirow{2}{*}{$\begin{array}{l}\text { Thickness } \\
\text { (feet) }\end{array}$} & \multicolumn{5}{|c|}{ Chemical analyses (percent) } & \multirow{2}{*}{$\begin{array}{l}\text { Cumulative } \\
\text { thickness } \\
\text { (feet) }\end{array}$} & \multirow{2}{*}{$\begin{array}{l}\text { Thickness } \mathrm{x} \\
\text { percent } \mathrm{P}_{2} \mathrm{O}_{5} \\
\text { (cumulative) }\end{array}$} & \multicolumn{2}{|c|}{$\begin{array}{l}\text { Uranium content } \\
\text { (percent) }\end{array}$} & \multirow{2}{*}{$\begin{array}{c}\text { Thickness } \mathrm{x} \\
\text { percent chem. } \\
\text { (cumulative) }\end{array}$} \\
\hline & & & & $\mathrm{P}_{2} \mathrm{O}_{5}$ & $\mathrm{Al}_{2} \mathrm{O}_{3}$ & $\mathrm{Fe}_{2} \mathrm{O}_{3}$ & $\begin{array}{l}\text { Loss on } \\
\text { ignition }\end{array}$ & $\begin{array}{l}\text { Acid } \\
\text { insoluble }\end{array}$ & & & $\frac{\operatorname{pe}}{\mathrm{eU}}$ & cent) & \\
\hline$P-143$ & Phosphate rock -...... & 4137-JDW & 0.8 & 34.4 & 1.3 & 0.72 & 2.88 & 8.6 & 32.1 & 256.66 & 0.014 & 0.014 & 0.117 \\
\hline $\mathrm{P}-142$ & Phosphate rock, argillaceous - & 4136-JDW & .5 & 27.8 & 3.0 & 1.15 & 4.22 & 22.1 & & 270.56 & .011 & .012 & .123 \\
\hline $\mathrm{P}-141$ & Mudstone, phosphatic & 4135-JDW & .5 & 10.9 & 7.9 & 3.97 & 5.40 & 56.9 & 33.1 & 276.01 & .005 & .006 & .126 \\
\hline $\mathrm{P}-140$ & Phosphate rock & $4134-J D W$ & .65 & 35.6 & 1.0 & .62 & 3.97 & 4.0 & 33.75 & 299.15 & .016 & .021 & .139 \\
\hline$P-139$ & Mudstone, phosphatic & $4133-\mathrm{JDW}$ & .6 & 14.2 & 6.6 & 2.63 & 5.77 & 50.2 & 34.35 & 307.67 & .007 & .005 & .142 \\
\hline $\mathrm{P}-138$ & Phosphate rock - & $4132-J D W$ & 1.9 & 31.2 & 2.1 & .79 & 5.67 & 12.2 & 36.25 & 366.95 & .014 & .013 & .167 \\
\hline $\mathrm{P}-137$ & Mudstone, phosphatic--- & $4131-J D W$ & 1.2 & 9.5 & 3.2 & 2.94 & 6.21 & 63.0 & 37.45 & 378.35 & .003 & .003 & .171 \\
\hline $\mathrm{P}-136$ & Mudstone and phosphate roc & $4130-J D W$ & 1.1 & 12.0 & 6.8 & 2.62 & 6.15 & 56.7 & 38.55 & 391.55 & .005 & .006 & .177 \\
\hline$P-135$ & Phosphate rock & $4129-J D W$ & .9 & 33.2 & 1.5 & .67 & 5.12 & 9.1 & 39.45 & 421.43 & .012 & .019 & .194 \\
\hline $\mathrm{P}-134$ & Phosphate rock, argillaceous & $4128-J D W$ & .65 & 26.9 & 4.3 & 1.80 & 5.75 & 20.7 & 40.10 & 438.92 & .007 & .012 & .202 \\
\hline P-133 & Phosphate rock -.. & 4127-JDW & 2.1 & 36.6 & .85 & .45 & 5.13 & 2.3 & 42. 20 & 515.78 & .016 & .023 & .250 \\
\hline $\mathrm{P}-132$ & Phosphate rock - & $4126-J D W$ & 4.0 & 26.1 & 2.90 & 1.22 & 13.70 & 14.2 & 46.20 & 620.18 & .013 & .016 & .314 \\
\hline$P-131$ & Phosphate rock, argillaceous & $4125-J D W$ & 5.0 & 24.1 & 3.5 & 1.66 & 14.66 & 18.2 & 51.20 & 740.68 & .010 & .012 & .374 \\
\hline $\mathrm{P}-130$ & Mudstone, phosphatic and mudstone - & $4124-J D W$ & 3.7 & 13.9 & -- & -- & -- & 39.8 & 54.90 & 792.10 & .006 & .008 & .404 \\
\hline$P-129$ & Mudstone - & $4123-J D W$ & 1.45 & 7.3 & -- & -- & -- & 64.6 & 56. 35 & 802.69 & .003 & .004 & .410 \\
\hline $\mathrm{P}-128$ & Mudstone - & 4538-MET & 1.1 & 2.8 & -- & -- & -. & 76.5 & 57.45 & 805.77 & .003 & .002 & .412 \\
\hline$P-127$ & Mudstone, phosphatic & 4537-MET & & 14.6 & -. & -- & -- & 40.0 & 59 & 830 & .007 & .006 & .422 \\
\hline $\mathrm{P}-126$ & Mudstone, phosphatic & 4536-MET & 1.1 & 14.1 & -- & -- & -- & 41.2 & 60. & 846.10 & .007 & .008 & .431 \\
\hline $\mathrm{P}-125$ & Mudstone, phosphatic & 4535-MET & 2.2 & 12.0 & -- & -- & -- & 49. & 62. & 872.50 & .006 & .005 & .442 \\
\hline$P-124$ & Mudstone, phosphatic -............ & 4534-MET & 2.3 & 11.3 & -- & -- & -- & 56.0 & 64.75 & 898.49 & .004 & .004 & .451 \\
\hline P-123 & Phosphate roc & 4533-MET & 2.3 & 20.9 & -- & -- & - & 37.5 & 67.05 & 946.56 & .004 & .006 & .465 \\
\hline $\mathrm{P}-122$ & Mudstone - & 4532-MET & 1.4 & 6.0 & -- & - & -- & 71.4 & 68.45 & 954.96 & .001 & .002 & .468 \\
\hline P-121 & Mudstone & 4531-MET & 1,2 & 5.5 & -- & -- & -- & 75.0 & 69. & 961.56 & .003 & .001 & .469 \\
\hline$P-120$ & Mudstone, phosphatic & 4530-MET & 2.2 & 14.5 & -- & -- & -- & 50.9 & & 993. & .005 & .005 & .480 \\
\hline$P-119$ & Mudstone, phosphatic & 4529-MET & 4.2 & 8.0 & -- & -- & -- & & 76.05 & $1,027.06$ & .003 & .001 & .484 \\
\hline$P-118$ & Mudstone & 4528-MET & 1.6 & 6.4 & -. & -. & -- & 72.6 & 77.65 & $1,037.30$ & .004 & .003 & .489 \\
\hline $\mathrm{P}-117$ & Mudstone and phosphate rock & 4527-MET & 2. & 11.8 & -- & -- & -- & 57.6 & 80.05 & 1,0 & .005 & .004 & .499 \\
\hline$P-116$ & Mudstone, phosphatic- & 4526-MET & 2.2 & 9.2 & -- & -- & -- & 57.1 & 82. 25 & $1,085.86$ & .003 & .002 & .503 \\
\hline$P-115$ & Mudstone & 4160 - RPS & 1.4 & 4.1 & -- & -- & -- & 72.9 & 83.65 & $1,091.60$ & .004 & .002 & .506 \\
\hline $\mathrm{P}-114$ & Mudstone - & 4159-RPS & .7 & 2.6 & -- & - & -- & & & $1,093.42$ & .002 & .001 & .506 \\
\hline$P-113$ & Mudstone - & 4158-RPS & 1.5 & 5.8 & -- & -- & $=$ & 64.7 & 85.85 & $1,102.11$ & .004 & .002 & .509 \\
\hline $\mathrm{P}-112$ & Mudstone - & 4157-RPS & 3.5 & 3.5 & -- & -- & -- & 75.1 & 89.35 & 1,1 & .003 & .001 & .513 \\
\hline P-111 & Mudstone, carbonatic - & 4156-RPS & 1.2 & 2.0 & -- & -- & -- & 59.2 & 90.55 & $1,116.76$ & .004 & .002 & .515 \\
\hline$P-110$ & Mudstone, phosphatic and phosphate & 4155- RPS & 1.0 & 19.9 & -- & -- & -- & 29.4 & 91.55 & 1,13 & .007 & .005 & .520 \\
\hline P-109 & Mudstone, phosphatic-. & 4154-RPS & 1.7 & 8.5 & -- & -- & -. & 60.5 & 93.25 & $1,151.11$ & .004 & .003 & .525 \\
\hline
\end{tabular}




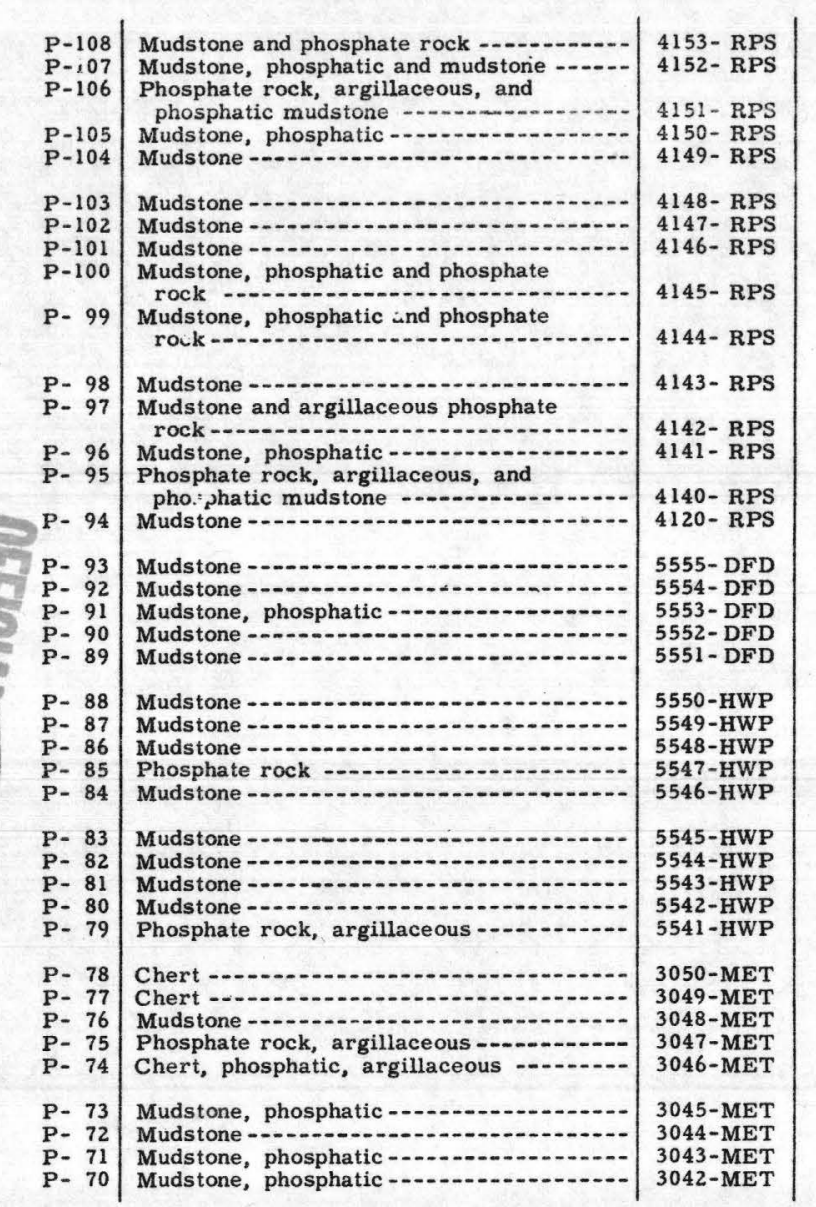

\begin{tabular}{|c|c|c|c|c|}
\hline $\begin{array}{l}2.4 \\
1.8\end{array}$ & $\begin{array}{l}\text { 8. } 0 \\
\text { 8. } 1\end{array}$ & $=$ & $=$ & $=$ \\
\hline $\begin{array}{l}1.2 \\
1.3\end{array}$ & $\begin{array}{l}18.2 \\
12.0\end{array}$ & $\overline{-}$ & $\therefore$ & $=$ \\
\hline 2.4 & 5.9 & -- & $\cdots$ & -- \\
\hline 2.6 & 4.2 & -. & -- & - \\
\hline $\begin{array}{r}.6 \\
1.8\end{array}$ & $\begin{array}{r}.6 \\
5.0\end{array}$ & $=$ & $\bar{z}$ & -- \\
\hline 1.6 & 11.5 & -- & -- & -. \\
\hline 1.9 & 21.8 & -. & -- & -. \\
\hline 1.6 & 2. 7 & -- & -- & .. \\
\hline $\begin{array}{l}1.4 \\
1.5\end{array}$ & $\begin{array}{r}13.5 \\
8.8\end{array}$ & $=$ & $=$ & $=$ \\
\hline $\begin{array}{l}1.4 \\
1.2\end{array}$ & $\begin{array}{r}16.6 \\
4.2\end{array}$ & $=$ & $=$ & $=$ \\
\hline .9 & 6.5 & - & -- & $=$ \\
\hline $\begin{array}{l}.7 \\
.4\end{array}$ & $\begin{array}{r}2.4 \\
13.2\end{array}$ & $\cdots$ & $\because$ & $\because$ \\
\hline 1.7 & 1.6 & $\cdots$ & - & $\because$ \\
\hline & & 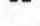 & 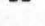 & \\
\hline 1.4 & 5.3 & - & -- & - \\
\hline 1.4 & $\begin{array}{r}.5 \\
4\end{array}$ & $\because$ & $\because-$ & $\because$ \\
\hline 1.5 & 29.1 & - & -. & - \\
\hline 1.3 & 2.9 & $\cdots$ & -- & -- \\
\hline 2.5 & 1.1 & - & -- & -. \\
\hline & .5 & - & -- & $\cdots$ \\
\hline 1.6 & $\begin{array}{r}.3 \\
1.4\end{array}$ & 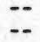 & $\because$ & $\ldots$ \\
\hline 1.5 & 18.6 & $\cdots$ & - & - \\
\hline 3.3 & .7 & -- & -- & - \\
\hline 3.4 & 2. 6 & 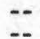 & $=$ & $\overline{-}$ \\
\hline $\begin{array}{r}1.6 \\
.8\end{array}$ & 17.8 & -- & - & -. \\
\hline 2.2 & 24.4 & -. & $\cdots$ & $\cdots$ \\
\hline 1.0 & 9.1 & - & -- & $a$ \\
\hline .8 & 1.2 & $=$ & $=$ & $=$ \\
\hline $\begin{array}{l}2.3 \\
1.1\end{array}$ & $\begin{array}{r}7.5 \\
12.6\end{array}$ & $\ldots$ & $\ldots$ & $\ldots$ \\
\hline
\end{tabular}

\begin{tabular}{|c|c|}
\hline $\begin{array}{l}51.2 \\
56.8\end{array}$ & $\begin{array}{l}95.65 \\
97.45\end{array}$ \\
\hline $\begin{array}{l}31.1 \\
47.7 \\
63.5\end{array}$ & $\begin{array}{r}98.65 \\
99.95 \\
102.35\end{array}$ \\
\hline $\begin{array}{l}72.2 \\
83.5 \\
73.6\end{array}$ & $\begin{array}{l}104.95 \\
105.55 \\
107.35\end{array}$ \\
\hline 45.2 & 108.95 \\
\hline 28.4 & 110.85 \\
\hline 76.4 & 112.45 \\
\hline $\begin{array}{l}41.6 \\
51.2\end{array}$ & $\begin{array}{l}113.85 \\
115.35\end{array}$ \\
\hline $\begin{array}{l}30.7 \\
69.5\end{array}$ & $\begin{array}{l}116.75 \\
117.95\end{array}$ \\
\hline $\begin{array}{l}60.7 \\
78.3 \\
55.2 \\
79.9 \\
70.5\end{array}$ & $\begin{array}{l}118.85 \\
119.55 \\
119.95 \\
121.65 \\
122.45\end{array}$ \\
\hline $\begin{array}{l}76.6 \\
87.7 \\
88.3 \\
17.3 \\
81.1\end{array}$ & $\begin{array}{l}123.85 \\
125.25 \\
125.75 \\
127.25 \\
128.55\end{array}$ \\
\hline $\begin{array}{l}84.6 \\
87.8 \\
87.8 \\
84.3 \\
41.4\end{array}$ & $\begin{array}{l}131.05 \\
131.85 \\
133.45 \\
133.95 \\
135.45\end{array}$ \\
\hline $\begin{array}{l}86.1 \\
81.6 \\
87.1 \\
40.1 \\
28.1\end{array}$ & $\begin{array}{l}138.75 \\
142.15 \\
143.75 \\
144.55 \\
146.75\end{array}$ \\
\hline $\begin{array}{l}58.4 \\
84.9 \\
61.8 \\
53.1\end{array}$ & $\begin{array}{l}149.75 \\
148.55 \\
150.85 \\
151.95\end{array}$ \\
\hline
\end{tabular}

\begin{tabular}{l|l}
$1,170.31$ & .004 \\
$1,184.89$ & .004 \\
$1,206.73$ & .006 \\
$1,222.33$ & .004 \\
$1,236.49$ & .002 \\
$1,247.41$ & .003 \\
$1,247.77$ & .002 \\
$1,256.77$ & .004 \\
$1,275.17$ & .004 \\
$1,316.59$ &. .007 \\
$1,320.91$ & .004 \\
$1,339.81$ & .005 \\
$1,353.01$ & .005 \\
$1,376.25$ & .005 \\
$1,381.29$ & .003 \\
$1,387.14$ & .004 \\
$1,388.82$ & .002 \\
$1,394.10$ & .003 \\
$1,396.82$ & .003 \\
$1,402.74$ & .004 \\
$1,410.16$ & .003 \\
$1,410.86$ & .002 \\
$1,411.06$ & .002 \\
$1,454.71$ & .006 \\
$1,458.48$ & .003 \\
$1,461.23$ & .002 \\
$1,461.63$ & .002 \\
$1,462.11$ & .002 \\
$1,462.81$ & .002 \\
$1,490.71$ & .008 \\
$1,493.02$ & .003 \\
$1,501.86$ & .002 \\
$1,502.98$ & .002 \\
$1,517.22$ & .005 \\
$1,570.90$ & .005 \\
$1,580.00$ & .005 \\
$1,580.96$ & .002 \\
$1,602.81$ & .003 \\
$1,616.67$ & .003
\end{tabular}

\begin{tabular}{l|l}
.002 & .530 \\
.004 & .537 \\
.005 & .543 \\
.003 & .547 \\
.003 & .555 \\
.003 & .562 \\
.002 & .564 \\
.003 & .569 \\
.005 & .577 \\
.006 & .588 \\
.002 & .592 \\
.005 & .599 \\
.004 & .605 \\
.006 & .613 \\
.002 & .615 \\
.004 & .619 \\
.002 & .620 \\
.002 & .621 \\
.001 & .623 \\
.002 & .624 \\
.002 & .627 \\
.001 & .629 \\
.001 & .629 \\
.006 & .638 \\
.002 & .641 \\
.001 & .643 \\
.001 & .644 \\
.001 & .646 \\
.001 & .646 \\
.007 & .657 \\
.001 & .666 \\
.001 & .663 \\
.001 & .665 \\
0.006 & .670 \\
.005 & .681 \\
.004 & .685 \\
.001 & .686 \\
.004 & .695 \\
.004 & .699 \\
&
\end{tabular}


South Stewart Canyon-Continued

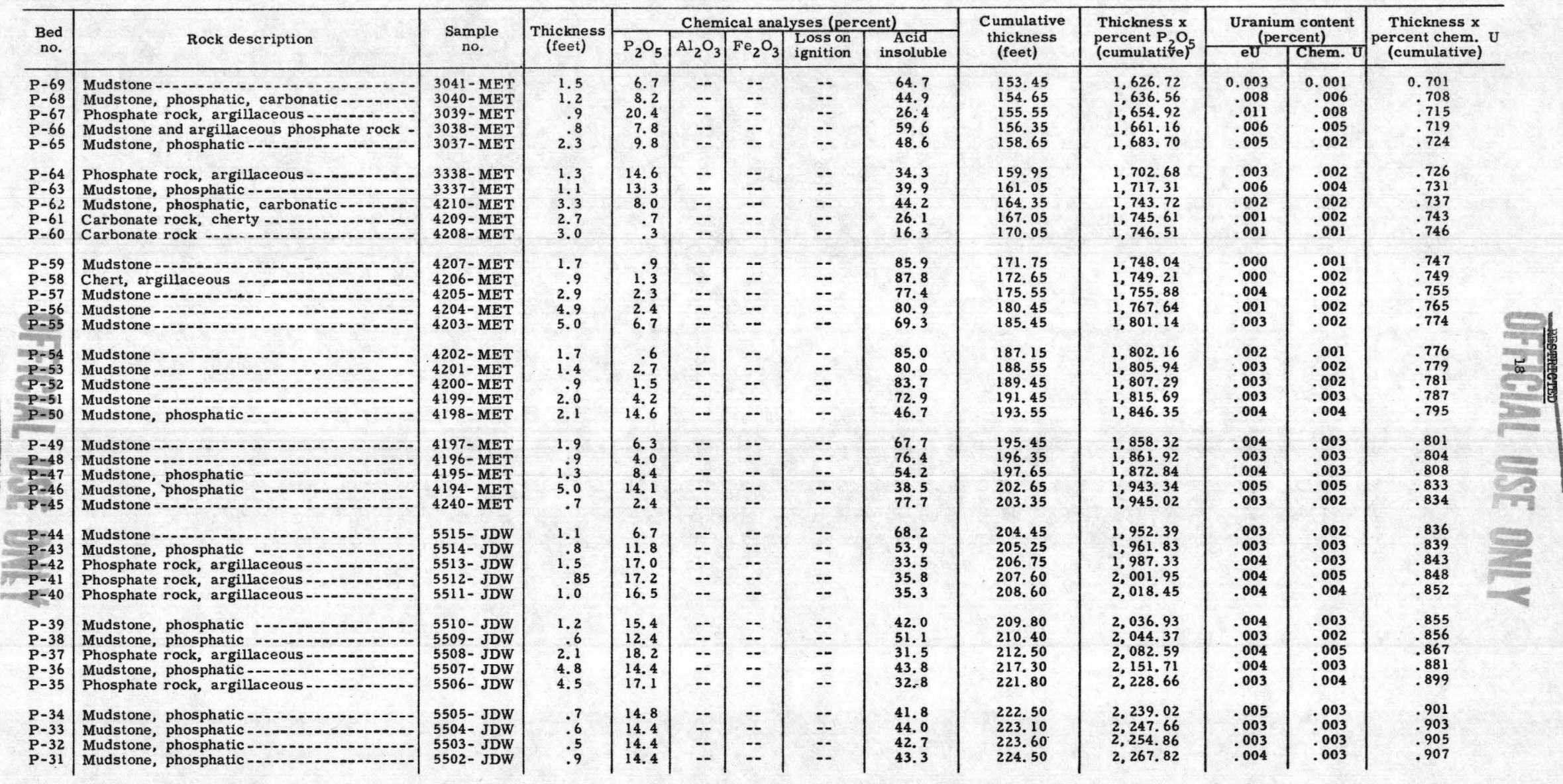

Lot 1306

IV - Idaho - 8 


\begin{tabular}{|c|c|c|c|c|c|c|c|c|c|c|c|c|c|c|c|}
\hline & P-30 & Phosphate rock, argillaceous & $5501-J D W$ & 1.4 & 17.6 & -- & -- & -- & 34.7 & 225.90 & $2,292.46$ & .004 & .004 & .913 & \\
\hline & $\begin{array}{c}P-29 \\
P-28\end{array}$ & Phosphate rock, argillaceous & $5500-$ JDW & 3.5 & 17.6 & & & & 36.8 & 229.40 & $2,354.06$ & .004 & .004 & .927 & \\
\hline & $\begin{array}{c}\text { P-28 } \\
\text { P-27 }\end{array}$ & Phosphate rock, argillaceous - & 5525- JDW. & 3.8 & 20.6 & 4.8 & 2.09 & 14. 34 & 22.8 & 233.20 & $2,432.34$ & .004 & .005 & .946 & \\
\hline & $\begin{array}{l}P-27 \\
P-26\end{array}$ & $\begin{array}{l}\text { Mudstone, phosphatic } \\
\text { Mudstone, phosphatic }\end{array}$ & $\begin{array}{l}5524-J \mathrm{JWW} \\
5523-\mathrm{JDW}\end{array}$ & $\begin{array}{l}1.5 \\
1.5\end{array}$ & $\begin{array}{l}13.2 \\
11.2\end{array}$ & $\begin{array}{l}7.4 \\
7.2\end{array}$ & $\begin{array}{l}3.13 \\
3.22\end{array}$ & $\begin{array}{r}9.59 \\
10.11\end{array}$ & $\begin{array}{l}44.9 \\
49.9\end{array}$ & $\begin{array}{l}234.70 \\
236.20\end{array}$ & $\begin{array}{l}2,452.14 \\
2,468.94\end{array}$ & .005 & .004 & .952 & \\
\hline & $\mathrm{P}-25$ & Phosphate rock-................. & 4122-JDW & 1.5 & 32.9 & 1.3 & 1.19 & & & & & & & & \\
\hline & $\mathrm{P}-24$ & Phosphate rock- & $4121-J D W$ & 1.0 & 27.4 & 1.7 & 1.17 & 12.04 & $\begin{array}{l}3.4 \\
7.3\end{array}$ & 238.70 & $\begin{array}{l}2,518.29 \\
2,545.69\end{array}$ & .006 & .009 & .974 & \\
\hline & $\mathrm{P}-23$ & Carbonate rock, argillaceous & 4107-MAW & $\because 9$ & 2.4 & .91 & 1.18 & 8.24 & 33.6 & 239.60 & $2,547.85$ & .006 & .009 & .988 & \\
\hline & $P-22$ & Carbonate rock, argillaceous, phosphatic -- & 4106-MAW & 1.1 & 8.6 & 4.5 & 1.89 & 25.93 & 23.0 & 240.70 & $2,557.31$ & .003 & .003 & .991 & \\
\hline & $P-21$ & Phosphate rock, argillaceous & 4105-MA,W & .6 & 21.2 & 4.4 & 1.98 & 10.19 & 24.4 & 241.30 & $2,570.03$ & .008 & .009 & .996 & \\
\hline & $\begin{array}{l}P-20 \\
P-19\end{array}$ & Carbonate rock, argillaceous & 4104-MAW & .7 & 3.7 & 3.7 & 1.45 & 33.61 & 19.1 & 242.00 & $2,572.62$ & .002 & .002 & .998 & \\
\hline & $\begin{array}{l}P-19 \\
P-18\end{array}$ & $\begin{array}{l}\text { Phosphate rock, argillaceous } \\
\text { Phosphate rock }\end{array}$ & 4103-MAW & .6 & 26.8 & 2.5 & 1.10 & 8.29 & 16.4 & 242.60 & $2,588,70$ & .009 & .011 & 1.004 & \\
\hline & $\mathrm{P}-17$ & $\begin{array}{l}\text { Phosphate rock- } \\
\text { Phosphate rock, argillaceous }\end{array}$ & $\begin{array}{l}\text { 4102-MAW } \\
\text { 4101-MAW }\end{array}$ & $\begin{array}{l}2.8 \\
3.3\end{array}$ & $\begin{array}{l}28.1 \\
20.0\end{array}$ & 2.6 & 1.22 & 7.81 & 12.5 & 245.40 & $2,667.38$ & .012 & .015 & 1.046 & \\
\hline & $\mathrm{P}-16$ & & 4100-MAW & 1.8 & $\begin{array}{r}20.0 \\
2.4\end{array}$ & $\begin{array}{l}4.3 \\
1.7\end{array}$ & $\begin{array}{r}1 . .91 \\
.67\end{array}$ & $\begin{array}{r}9.75 \\
37.57\end{array}$ & $\begin{array}{l}30.2 \\
13.5\end{array}$ & $\begin{array}{l}248.70 \\
250.50\end{array}$ & $\begin{array}{l}2,733.38 \\
2,737.70\end{array}$ & $\begin{array}{l}.009 \\
.002\end{array}$ & .013 & $\begin{array}{l}1.089 \\
1.091\end{array}$ & \\
\hline & P-15 & Mudstone, phosphatic & $5522-J D W$ & 2.5 & 11.8 & 6.6 & .24 & 9.79 & 45.3 & 253.00 & $2,767.20$ & .005 & .005 & 1.103 & \\
\hline & P-14 & Carbonate rock, argillaceous & $5521-J D W$ & 1.0 & 2.1 & 2.8 & 1.05 & 34.54 & 21.2 & 254.00 & $2,769.30$ & .001 & .002 & 1.105 & \\
\hline & $\begin{array}{l}P-13 \\
P-12\end{array}$ & Phosphate rock, argillaceous ............ & $5520-J D W$ & (?) & 24.6 & 2.5 & 1.04 & 10.26 & 18.0 & $=$ & & .007 & .008 & & \\
\hline & & $\begin{array}{l}\text { Interval, probably small, between beds } \\
\text { P-13 and } \mathbf{P}-12 \text {. }\end{array}$ & & & & & & & & & & & & & \\
\hline$=$ & P-12 & Phosphate rock, argillaceous ........... & 4525-RGW & .8 & 18.5 & 4.3 & 3.34 & 10.29 & 32.7 & $* .8$ & *14.80 & .010 & .009 & $* .007$ & \\
\hline & $P-11$ & Mudstone, phosphatic .................. & 4524-RGW & .9 & 14.6 & 6.2 & 1.85 & 10.65 & 41.9 & 1.7 & 27.94 & .007 & .007 & .014 & 0 \\
\hline 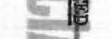 & $\begin{array}{r}P-10 \\
P-9\end{array} \mid \quad-190$ & Phosphate rock & 4523-RGW & 1.6 & 29.6 & 2.2 & .68 & 7.82 & 12.5 & 3.3 & 75.30 & .009 & .015 & .038 & \\
\hline $3=$ & $\begin{array}{l}P-9 \\
P-8\end{array}$ & $\begin{array}{l}\text { Phosphate rock } \\
\text { Phosphate rock, argillaceous }\end{array}$ & $\begin{array}{l}4522-\mathrm{RGW} \\
4521-\mathrm{RGW}\end{array}$ & $\begin{array}{r}1.7 \\
.7\end{array}$ & $\begin{array}{l}30.3 \\
27.4\end{array}$ & $\begin{array}{l}1.9 \\
2.7\end{array}$ & $\begin{array}{l}.67 \\
.78\end{array}$ & $\begin{array}{l}7.56 \\
7.15\end{array}$ & $\begin{array}{l}11.8 \\
18.0\end{array}$ & $\begin{array}{l}5.0 \\
5.7\end{array}$ & $\begin{array}{l}126.81 \\
145.99\end{array}$ & $\begin{array}{l}.009 \\
.008\end{array}$ & $\begin{array}{l}.012 \\
.013\end{array}$ & $\begin{array}{l}.058 \\
.067\end{array}$ & 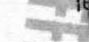 \\
\hline & P- 7 & Phosphate rock- & 4511-RGW & 1.2 & 31.1 & .92 & .53 & 8.04 & 5.0 & 6.9 & 183.31 & .019 & .023 & .095 & 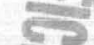 \\
\hline$=$ & $P-6$ & Phosphate rock, argillaceous -........ & 4244-MAW & .7 & 24.3 & 2.7 & 1.30 & 8.93 & 19.7 & 7.6 & 200.32 & .010 & .012 & .103 & 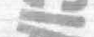 \\
\hline 20 & P- 5 & Mudstone $-\ldots$ & 4243-MAW & .6 & 1.5 & 7.7 & 3.95 & 6.24 & $75 . ?$ & 8.2 & 201.22 & .002 & .002 & .104 & Deter \\
\hline $3 \frac{1}{1}$ & $\begin{array}{r}P-4 \\
P-3\end{array}$ & Mudstone, phosphatic, carbonatic ....... & 4242-MAW & 1.0 & 9.6 & 6.2 & 2.37 & 11.12 & 44.5 & 9.2 & 210.82 & .004 & .004 & .108 & r. \\
\hline $\operatorname{aratg}$ & $\begin{array}{l}\text { P- } 3 \\
\text { P- } 2\end{array}$ & Mudstone, carbonatic - & 4241-MAW & .7 & 2.4 & 5.7 & 2.05 & 19.29 & 45.2 & 9.9 & 212.50 & .002 & .002 & .110 & $\Rightarrow$ \\
\hline 2 & $\begin{array}{l}\text { P- } \\
\text { P- } 1\end{array}$ & $\begin{array}{l}\text { Limestone } \\
\text { Phosphate rock- }\end{array}$ & $\begin{array}{l}4433-D F D \\
4434-D F D\end{array}$ & $\begin{array}{l}3.8 \\
1.6\end{array}$ & $\begin{array}{r}3.25 \\
35.5\end{array}$ & $\overline{-}$ & $\overline{-}$ & $\ddot{--}$ & $\begin{array}{l}4.0 \\
3.3\end{array}$ & $\begin{aligned} 13.7 \\
* * 15.3\end{aligned}$ & $\begin{array}{r}224.85 \\
* * 281.65\end{array}$ & .001 & $\begin{array}{l}.002 \\
.012\end{array}$ & $\begin{array}{r}.117 \\
* .136\end{array}$ & 28 \\
\hline m & & & Well & ormati & - top b & ed only & & & & & & & & & \\
\hline & $\mathrm{Cw}-1$ & Limestone, siliceous & -- & (?) & -- & -. & -. & -- & -. & -- & -. & -- & -- & $\cdots$ & $=$ \\
\hline & & mulative & & & & & & & & & & & & & $\sin$ \\
\hline
\end{tabular}


Pole Canyon, Idaho, lot 1303

Phosphatic shale member of the Phosphoria formation sampled in bulldozer trench on the west limb of the Boulder Creek anticline, in Pole Canyon,

SW $\frac{1}{4}$ sec. 31, T. 8 S., R. 46 E., Caribou County, Idaho. Beds strike N. $10^{\circ} \mathrm{W}$. and dip $20^{\circ} \mathrm{W}$. Section measured and sampled by R. A. Smart, F. J. Anders on, R. S. Jones, and R. G. Waring in August 1949. Samples analyzed for $\mathrm{P}_{2} \mathrm{O}_{5}$ and acid in soluble by U. S. Bureau of
Mines laboratory, Albany, Oreg., and for other constituents by Trace Elements Section laboratory, U. S. Geological Survey, Washington, D. C.
Samples analyzed for eU and chem. U by the U. S. Geological Survey aboratory, Geochemistry and

\begin{tabular}{|c|c|c|c|c|c|c|c|c|c|c|c|c|}
\hline \multirow{2}{*}{$\begin{array}{c}\text { Bed } \\
\text { no. }\end{array}$} & \multirow{2}{*}{ Rock description } & \multirow{2}{*}{$\begin{array}{c}\text { Sample } \\
\text { no. }\end{array}$} & \multirow{2}{*}{$\begin{array}{c}\text { Thickness } \\
\text { (feet) }\end{array}$} & \multicolumn{5}{|c|}{ Chemical analyses (percent) } & \multirow{2}{*}{$\begin{array}{l}\text { Cumulative } \\
\text { thickness } \\
\text { (feet) }\end{array}$} & \multirow{2}{*}{$\begin{array}{l}\text { Thickness } x \\
\text { percent } P_{2} O_{5} \\
\text { (cumulative) }\end{array}$} & \multirow{2}{*}{$\begin{array}{l}\begin{array}{c}\text { Uranium content } \\
\text { (percent) }\end{array} \\
\begin{array}{c|c|c|}\text { eU } & \text { Chem. U }\end{array}\end{array}$} & \multirow{2}{*}{$\begin{array}{l}\text { Thickness } x \\
\text { percent eU } \\
\text { (cumulative) }\end{array}$} \\
\hline & & & & $\mathrm{P}_{2} \mathrm{O}_{5}$ & $\mathrm{Al}_{2} \mathrm{O}_{3}$ & $\mathrm{Fe}_{2} \mathrm{O}_{3}$ & $\begin{array}{l}\text { Loss on } \\
\text { ignition }\end{array}$ & $\begin{array}{l}\text { Accid } \\
\text { insoluble }\end{array}$ & & & & \\
\hline
\end{tabular}

\begin{tabular}{|c|c|c|c|c|c|c|c|c|c|c|c|c|c|}
\hline R- 1 & Chert - & -- & 4.0 & -- & -- & -- & -- & -- & 4.0 & -- & -- & -- & -- \\
\hline
\end{tabular}

Phosphatic shale member of Phosphoria formation

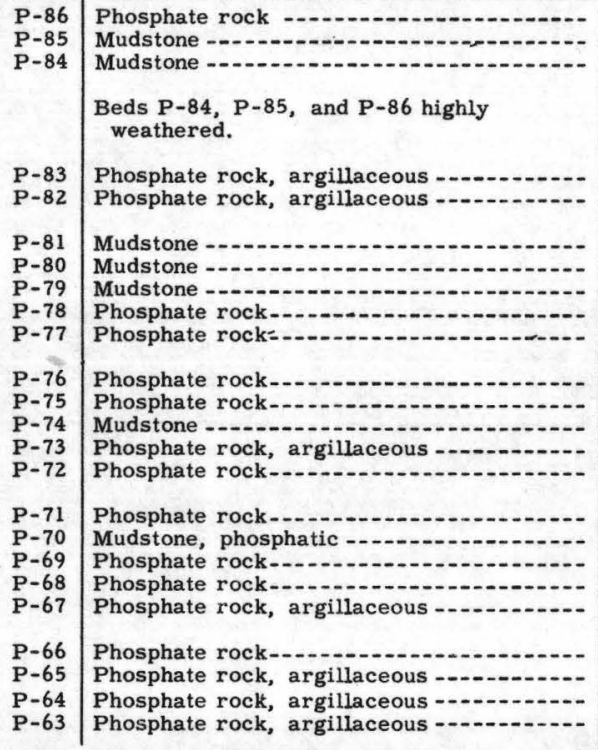

Lot 1303

4225-MAW
$4224-$ MAW

$4223-$ MAW
$4222-$ MAW
$4221-$ MAW
$4432-$ RAS
$4431-$ RAS
$4430-$ RAS
$4429-$ RAS
$4428-$ RAS
$4427-$ RAS
$4426-$ RAS
$4425-$ RAS
$4424-$ RAS
$4423-$ RAS
$4422-$ RAS
$4421-$ RAS
$4420-$ RAS
$4419-$ RAS
4418- RAS
$4417-$ RAS
$4416-$ RAS
4415- RAS

\begin{tabular}{r|r}
0.2 \\
1.8 \\
3.4 \\
\\
\\
.7 \\
1.4 \\
1.5 \\
.9 \\
.8 \\
1.2 \\
.5 \\
.9 \\
1.3 \\
1.1 \\
1.1 \\
2.0 \\
1.0 \\
1.3 \\
1.7 \\
1.9 \\
1.2 \\
2.0 \\
1.4 \\
2.6 \\
2.2
\end{tabular}

\begin{tabular}{|r|c|c|c|}
\hline--.9 & -- & -- & -- \\
1.9 & -- & -- & -- \\
& & & \\
& & & \\
21.9 & -- & -- & -- \\
24.2 & -- & -- & -- \\
5.0 & -- & -- & -- \\
2.0 & -- & - & -- \\
6.1 &.- &.- & -- \\
35.3 & 1.1 & 0.44 & 5.06 \\
37.8 & .44 & .16 & 3.60 \\
34.6 & 1.4 & .67 & 7.04 \\
33.2 & 1.2 & .63 & 2.12 \\
6.7 & 9.7 & 3.69 & 5.26 \\
21.7 & 5.1 & 1.93 & 3.72 \\
34.6 & 1.3 & .56 & 2.44 \\
35.9 & 1.1 & .50 & 3.80 \\
17.2 & 5.6 & 2.37 & 5.44 \\
35.3 & 1.4 & .54 & 7.22 \\
35.4 & 1.3 & .45 & 5.42 \\
26.5 & 3.4 & 1.43 & 13.86 \\
30.3 & 2.0 & .88 & 11.64 \\
27.0 & 3.3 & 1.34 & 9.94 \\
24.0 & 3.4 & 1.61 & 13.62 \\
18.2 &.-- & -- & -- \\
\end{tabular}

0.2
77.7
68.8



32.8
27.7
69.2
78.1
67.6
4.0
2.3
4.4
13.5
66.6
34.8
9.0
4.7
43.6
5.8
4.5
15.8
8.5
16.5
21.0
32.5

0.2
2.0
5.4


6.1
7.5
9.0
9.9
10.7
11.9
12.4
13.3
14.6
15.7
16.8
18.8
19.8
21.1
22.8
24.7
25.9
27.9
29.3
31.9
34.1

.--42
3.42



35.75
69.63
77.13
78.93
83.81
126.17
145.07
176.21
219.37
226.74
250.61
319.81
355.71
378.07
438.08
505.34
537.14
597.74
635.54
697.94
737.98

$0 .-$
0.003
.008

.007
.009
.004
.002
.004
.011
.009
.010
.012
.004
.007
.014
.021
.006
.017
.020
.016
.019
.013
.008
.009

\begin{tabular}{c}
\hline- \\
0.002 \\
\\
\\
.005 \\
.008 \\
.- \\
.- \\
.010 \\
.010 \\
.011 \\
.011 \\
.007 \\
.013 \\
.022 \\
.004 \\
.017 \\
.018 \\
.017 \\
.019 \\
.011 \\
.008 \\
.007
\end{tabular}

0.005

.033

.038
.050

.056

.058

.061
.074
.079

088

.088
.103

.116

144

164
.172

.201

.239
.258

.296
.315

.315
.335
.355

IV - Idaho - 10 


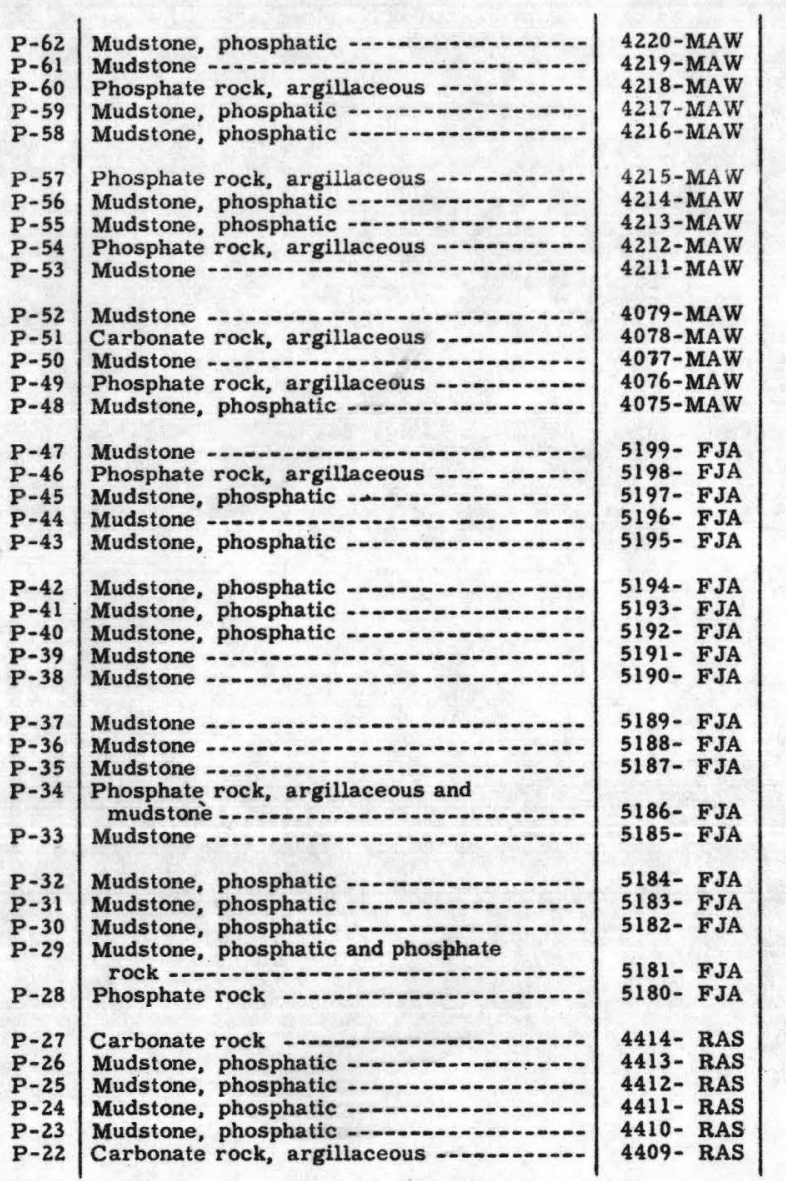

Lot 1303

\begin{tabular}{|c|c|c|c|c|}
\hline $\begin{array}{r}1.3 \\
1.4 \\
3.2 \\
2.6 \\
.9\end{array}$ & $\begin{array}{r}8.4 \\
3.0 \\
17.4 \\
13.4 \\
15.8\end{array}$ & $\begin{array}{l}\because \\
\bar{z} \\
=\end{array}$ & $\begin{array}{l}-\therefore \\
\therefore \\
\therefore \\
\therefore\end{array}$ & $\begin{array}{l}= \\
=- \\
=- \\
=\end{array}$ \\
\hline $\begin{array}{l}2.3 \\
1.5 \\
3.9 \\
1.8 \\
2.1\end{array}$ & $\begin{array}{r}18.8 \\
16.5 \\
9.5 \\
21.8 \\
5.1\end{array}$ & 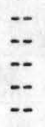 & $\begin{array}{l}-- \\
- \\
-\end{array}$ & $\begin{array}{l}\because- \\
\square \\
=- \\
-\end{array}$ \\
\hline $\begin{array}{l}1.2 \\
1.8 \\
2.8 \\
1.9 \\
2.0\end{array}$ & $\begin{array}{r}7.4 \\
7.1 \\
1.9 \\
19.2 \\
9.1\end{array}$ & $\begin{array}{l}= \\
\because- \\
\because- \\
-\end{array}$ & 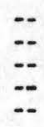 & $\begin{array}{l}\because- \\
\because- \\
\because-\end{array}$ \\
\hline $\begin{array}{l}1.9 \\
3.0 \\
1.0 \\
2.7 \\
1.1\end{array}$ & $\begin{array}{r}1.8 \\
18.8 \\
9.7 \\
5.8 \\
14.2\end{array}$ & $\begin{array}{l}= \\
\bar{z} \\
\ddot{z}\end{array}$ & $\begin{array}{l}z \\
z \\
z \\
z-\end{array}$ & $\begin{array}{l}\because- \\
\because- \\
\therefore \\
--\end{array}$ \\
\hline $\begin{array}{l}.7 \\
2.6 \\
3.6 \\
1.2 \\
2.1\end{array}$ & $\begin{array}{r}10.0 \\
13.4 \\
12.1 \\
5.4 \\
2.6\end{array}$ & 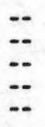 & $\begin{array}{l}z- \\
z- \\
z \\
z\end{array}$ & $\begin{array}{l}\because- \\
\because- \\
\because \\
-\end{array}$ \\
\hline $\begin{array}{l}2.4 \\
1.1 \\
1.6\end{array}$ & $\begin{array}{r}2.6 \\
2.4 \\
.4\end{array}$ & $\begin{array}{l}= \\
=-\end{array}$ & $\begin{array}{l}\because- \\
\because\end{array}$ & $\begin{array}{l}\because \\
\because\end{array}$ \\
\hline $\begin{array}{l}2.2 \\
1.4\end{array}$ & $\begin{array}{r}10.5 \\
1.6\end{array}$ & $=$ & $\overline{-}$ & $\because$ \\
\hline $\begin{array}{l}3.6 \\
1.8 \\
3.9\end{array}$ & $\begin{array}{r}9.9 \\
11.9 \\
14.8\end{array}$ & $\begin{array}{l}\because- \\
=\end{array}$ & $\begin{array}{l}\ldots \\
\therefore \\
-.\end{array}$ & -. \\
\hline $\begin{array}{l}3.7 \\
2.5\end{array}$ & $\begin{array}{l}17.9 \\
28.6\end{array}$ & $=$ & -- & $\because$ \\
\hline $\begin{array}{l}1.8 \\
1.9 \\
2.0\end{array}$ & $\begin{array}{r}2.4 \\
13.9 \\
13.4 \\
11.1 \\
10.1 \\
3.1\end{array}$ & $\begin{array}{l}=- \\
\because- \\
=- \\
=\end{array}$ & 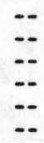 & $\begin{array}{l}\because \\
\because\end{array}$ \\
\hline
\end{tabular}

\begin{tabular}{l|l} 
& \\
58.9 & 35.4 \\
63.4 & 36.8 \\
33.7 & 40.0 \\
49.2 & 42.6 \\
42.7 & 43.5 \\
42.7 & 45.8 \\
46.0 & 47.3 \\
62.8 & 51.2 \\
29.8 & 53.0 \\
69.7 & 55.1 \\
55.2 & 56.3 \\
27.8 & 58.1 \\
79.0 & 60.9 \\
33.0 & 62.8 \\
66.4 & 64.8 \\
79.6 & 66.7 \\
41.7 & 69.7 \\
63.4 & 70.7 \\
69.7 & 73.4 \\
33.0 & 74.5 \\
56.6 & 75.2 \\
38.4 & 77.8 \\
50.1 & 81.4 \\
59.9 & 82.6 \\
74.0 & 84.7 \\
70.8 & 87.1 \\
78.3 & 88.2 \\
84.7 & 89.8 \\
56.3 & 92.0 \\
82.9 & 93.4 \\
54.4 & 97.0 \\
51.1 & 98.8 \\
38.7 & 102.7 \\
33.3 & 106.4 \\
12.1 & 108.9 \\
12.2 & 111.9 \\
45.3 & 113.9 \\
46.3 & 115.9 \\
50.3 & 117.7 \\
55.0 & 119 \\
28.3 & 121.6 \\
& \\
\hline
\end{tabular}

748.90
753.10
808.78
843.62
857.84
901.08
925.83
962.88
$1,002.12$
$1,012.83$
$1,021.71$
$1,034.49$
$1,039.81$
$1,076.29$
$1,094.49$
$1,097.91$
$1,154.31$
$1,164.01$
$1,179.67$
$1,195.29$
$1,202.29$
$1,237.13$
$1,280.69$
$1,287.17$
$1,292.63$
$1,298.87$
$1,301.51$
$1,302.15$
$1,325.25$
$1,327.49$
$1,363.13$
$1,384.55$
$1,442.27$
$1,508.50$
$1,580.00$
$1,587.20$
$1,612.22$
$1,641.70$
$1,661.68$
$1,680.87$
$1,687.07$

.004
.002
.007
.006
.006
.004
.005
.005
.006
.004
.004
.005
.003
.006
.004
.003
.005
.005
.004
.011
.010
.005
.004
.004
.003
.001
.002
.002
.004
.003
.004
.004
.004
.005
.008
.0005
.006
.007
.006
.005
.002

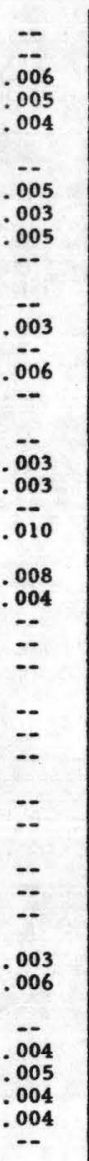


Pole Canyon-Continued

\begin{tabular}{|c|c|c|c|c|c|c|c|c|c|c|c|c|c|}
\hline \multirow{2}{*}{$\begin{array}{c}\text { Bed } \\
\text { no. }\end{array}$} & \multirow{2}{*}{ Rock description } & \multirow{2}{*}{$\begin{array}{c}\text { Sample } \\
\text { no. }\end{array}$} & \multirow{2}{*}{$\begin{array}{c}\text { Thickness } \\
\text { (feet) }\end{array}$} & \multicolumn{5}{|c|}{ Chemical analyses (percent) } & \multirow{2}{*}{$\begin{array}{l}\text { Cumulative } \\
\text { thickness } \\
\text { (feet) }\end{array}$} & \multirow{2}{*}{$\begin{array}{l}\text { Thickness X } \\
\text { percent } \mathbf{P}_{2} \mathrm{O}_{5} \\
\text { (cumulative) }\end{array}$} & \multirow{2}{*}{\multicolumn{2}{|c|}{$\begin{array}{c}\text { Uranium content } \\
\text { (percent) }\end{array}$}} & \multirow{2}{*}{$\begin{array}{l}\text { Thickness } \mathbf{x} \\
\text { percent eU } \\
\text { (cumulative) }\end{array}$} \\
\hline & & & & $\mathrm{P}_{2} \mathrm{O}_{5}$ & $\mathrm{Al}_{2} \mathrm{O}_{3}$ & $\mathrm{Fe}_{2} \mathrm{O}_{3}$ & $\begin{array}{l}\text { Loss on } \\
\text { ignition }\end{array}$ & $\begin{array}{c}\text { Acid } \\
\text { insoluble }\end{array}$ & & & & & \\
\hline $\begin{array}{l}P-21 \\
P-20 \\
P-19 \\
P-18 \\
P-17\end{array}$ & $\begin{array}{l}\text { Phosphate rock, argillaceous } \\
\text { Phosphate rock, argillaceous } \\
\text { Carbonate rock, argillaceous } \\
\text { Phosphate rock- } \\
\text { Phosphate rock }\end{array}$ & $\begin{array}{l}\text { 4408- RAS } \\
5166-\text { RSJ } \\
5164-\text { RSJ } \\
5163-\text { RSJ } \\
\text { 5162- RSJ }\end{array}$ & $\begin{array}{r}1.9 \\
2.1 \\
.9 \\
.9 \\
1.3\end{array}$ & $\begin{array}{l}27.0 \\
22.5 \\
1.1 \\
29.0 \\
29.6\end{array}$ & $\begin{array}{l}2.7 \\
4.0 \\
2.7 \\
2.1 \\
2.3\end{array}$ & $\begin{array}{r}1.19 \\
1.44 \\
.99 \\
.86 \\
1.01\end{array}$ & $\begin{array}{r}8.96 \\
7.56 \\
32.78 \\
8.00 \\
6.52\end{array}$ & $\begin{array}{l}16.7 \\
29.1 \\
21.9 \\
12.1 \\
14.3\end{array}$ & $\begin{array}{l}123.5 \\
125.6 \\
126.5 \\
127.4 \\
128.7\end{array}$ & $\begin{array}{l}1,738.37 \\
1,785.62 \\
1,786.61 \\
1,812.71 \\
1,851.19\end{array}$ & $\begin{array}{l}0.010 \\
.009 \\
.0005 \\
.014 \\
.011\end{array}$ & $\begin{array}{l}0.009 \\
.010 \\
.000 \\
.014 \\
.009\end{array}$ & $\begin{array}{r}0.765 \\
.784 \\
.785 \\
.797 \\
.811\end{array}$ \\
\hline $\begin{array}{l}P-16 \\
P-15 \\
P-14 \\
P-13 \\
P-12\end{array}$ & $\begin{array}{l}\text { Phosphate rock } \\
\text { Phosphate rock, argillaceous } \\
\text { Phosphate rock, contains carbonate rock lens } \\
\text { Carbonate rock, phosphatic-.......... } \\
\text { Phosphate rock, argillaceous, carbonatic -. }\end{array}$ & $\begin{array}{l}\text { 5161- RSJ } \\
5204-\text { RGW } \\
5203-\text { RGW } \\
5202-\text { RGW } \\
5201-\text { RGW }\end{array}$ & $\begin{array}{r}2.6 \\
1.0 \\
1.0 \\
.8 \\
.7\end{array}$ & $\begin{array}{l}31.4 \\
22.1 \\
26.2 \\
8.4 \\
16.7\end{array}$ & $\begin{array}{l}1.7 \\
4.2 \\
3.2 \\
1.3 \\
4.7\end{array}$ & $\begin{array}{l}.73 \\
1.33 \\
1.7 \\
1.58 \\
1.14\end{array}$ & $\begin{array}{r}6.87 \\
8.02 \\
7.70 \\
31.06 \\
16.56\end{array}$ & $\begin{array}{l}10.2 \\
27.8 \\
17.1 \\
10.4 \\
21.0\end{array}$ & $\begin{array}{l}131.3 \\
132.3 \\
133.3 \\
134.1 \\
134.8\end{array}$ & $\begin{array}{l}1,932.83 \\
1,954.93 \\
1,981.13 \\
1,987.85 \\
1,999.54\end{array}$ & $\begin{array}{l}.013 \\
.010 \\
.003 \\
.003 \\
.004\end{array}$ & $\begin{array}{l}.013 \\
.008 \\
-- \\
--\end{array}$ & $\begin{array}{l}.845 \\
.855 \\
.858 \\
.861 \\
.863\end{array}$ \\
\hline $\begin{array}{l}P-11 \\
P-10 \\
P-9 \\
P-8 \\
P-7\end{array}$ & $\begin{array}{l}\text { Phosphate rock, argillaceous } \\
\text { Phosphate rock, argillaceous } \\
\text { Phosphate rock- } \\
\text { Phosphate rock- } \\
\text { Phosphate rock }\end{array}$ & $\begin{array}{l}\text { 5200- RGW } \\
5160-\text { RGW } \\
5159-\text { RGW } \\
5158-\text { RGW } \\
5157-\text { RGW }\end{array}$ & $\begin{array}{l}1.8 \\
.6 \\
.9 \\
.7 \\
.8\end{array}$ & $\begin{array}{l}26.3 \\
24.4 \\
33.3 \\
34.2 \\
32.7\end{array}$ & $\begin{array}{l}3.3 \\
3.5 \\
1.0 \\
.67 \\
1.1\end{array}$ & $\begin{array}{r}1.17 \\
1.77 \\
.43 \\
.34 \\
.50\end{array}$ & $\begin{array}{l}6.48 \\
6.32 \\
6.74 \\
6.66 \\
7.04\end{array}$ & $\begin{array}{r}20.3 \\
24.5 \\
4.9 \\
3.3 \\
4.5\end{array}$ & $\begin{array}{l}136.6 \\
137.2 \\
138.1 \\
138.8 \\
139.6\end{array}$ & $\begin{array}{l}2,046.88 \\
2,061.52 \\
2,091.49 \\
2,115.43 \\
2,141.59\end{array}$ & $\begin{array}{l}.006 \\
.005 \\
.008 \\
.008 \\
.009\end{array}$ & $\begin{array}{l}.006 \\
.003 \\
.008 \\
.008 \\
.009\end{array}$ & $\begin{array}{l}.874 \\
.877 \\
.884 \\
.890 \\
.897\end{array}$ \\
\hline $\begin{array}{l}\mathrm{P}-6 \\
\mathrm{P}-5 \\
\mathrm{P}-4 \\
\mathrm{P}-3 \\
\mathrm{P}-2\end{array}$ & $\begin{array}{l}\text { Phosphate rock- } \\
\text { Phosphate rock } \\
\text { Mudstone - carbonatic and limestone } \\
\text { Mudstone, cork, argillaceous - } \\
\text { Carbonate rock, }\end{array}$ & $\begin{array}{l}\text { 5156- RGW } \\
5155-\mathrm{RGW} \\
5154-\mathrm{RGW} \\
5153-\mathrm{RGW} \\
5152-\mathrm{RGW}\end{array}$ & $\begin{array}{r}.9 \\
.9 \\
.7 \\
2.0 \\
1.1\end{array}$ & $\begin{array}{r}34.3 \\
35.6 \\
4.2 \\
.5 \\
1.2\end{array}$ & $\begin{array}{l}.68 \\
.55 \\
-: \\
--\end{array}$ & $\begin{array}{l}.41 \\
.31 \\
-: \\
-:\end{array}$ & $\begin{array}{c}5.68 \\
4.42 \\
-- \\
--\end{array}$ & $\begin{array}{r}2.5 \\
2.5 \\
66.7 \\
31.7 \\
39.8\end{array}$ & $\begin{array}{l}140.5 \\
141.4 \\
142.1 \\
144.1 \\
145.2\end{array}$ & $\begin{array}{l}2,172.46 \\
2,204.50 \\
2,207.44 \\
2,208.44 \\
2,209.76\end{array}$ & $\begin{array}{l}.029 \\
.014 \\
.005 \\
.001 \\
.002\end{array}$ & $\begin{array}{l}.029 \\
.014 \\
.003 \\
-- \\
--\end{array}$ & $\begin{array}{l}.923 \\
.936 \\
.939 \\
.941 \\
.944\end{array}$ \\
\hline-1 & Phosphate rock-...-. & 5151-RGW & .3 & 32.0 & -- & -- & -- & 5.2 & 145.5 & $2,219.36$ & .011 & .012 & .947 \\
\hline \multicolumn{14}{|c|}{ Wells formation - top bed only } \\
\hline $\mathrm{Cw}_{\mathrm{w}-1}$ & Carbonate rock- & $5150-$ RGW & 0.8 & 2.1 & - & 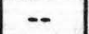 & -- & 4.2 & 0.8 & -- & $0: 002$ & -- & -- \\
\hline
\end{tabular}


Spectrographic Analyses - Pole Canyon, Idaho, lot 1303

Semiquantitative analyses of samples of the phosphatic shale member of the Phosphoria formation, Pole Canyon, Idaho (see immediately preceding pages for location of section, thickness and description of strata, and chemical analyses of samples), made by U. S. Geological Survey, Geochemistry and Petrology $\mathrm{Li}, \mathrm{Lu}, \mathrm{Hg}, \mathrm{Nd}, \mathrm{Os}, \mathrm{Pd}, \mathrm{Pt}, \mathrm{Pr}, \mathrm{Re}, \mathrm{Rh}, \mathrm{Rb}, \mathrm{Ru}, \mathrm{Sm}, \mathrm{Ta}, \mathrm{Te}, \mathrm{Tb}, \mathrm{Tl}$, Th, and $\mathrm{W}$ were looked for in all samples but were not detected

Explanation of Symbols

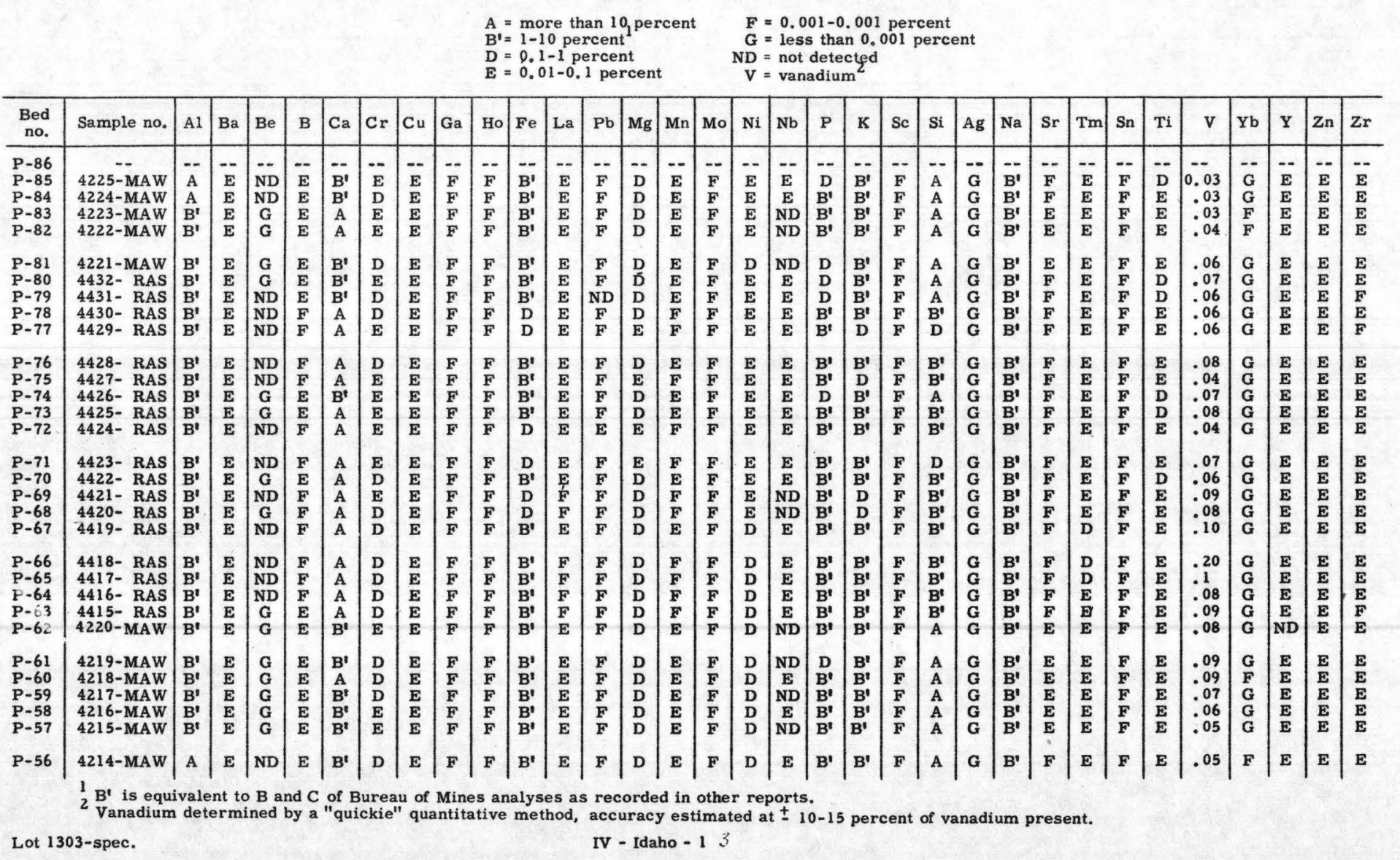


Spectrographic Analyses - Pole Canyon-Continued

\begin{tabular}{|c|c|c|c|c|c|c|c|c|c|c|c|c|c|c|c|c|c|c|c|c|c|c|c|c|c|c|c|c|c|c|c|c|c|}
\hline & & & & & & & & & & & & & & & & & & & & & & & & & & & & & & & & & \\
\hline & & & 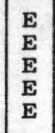 & $\begin{array}{l}\mathrm{G} \\
\mathrm{G} \\
\mathrm{G}\end{array}$ & 1 & 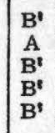 & & \begin{tabular}{|l}
$\mathrm{E}$ \\
$\mathrm{E}$ \\
$\mathrm{E}$ \\
$\mathrm{E}$
\end{tabular} & $\begin{array}{l}F \\
F \\
F\end{array}$ & . & & & & & & \begin{tabular}{|l}
$F$ \\
$F$ \\
$F$
\end{tabular} & $\mathrm{D}$ & 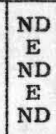 & $\vdots$ & : & $\begin{array}{l}\mathbf{F} \\
\mathbf{F} \\
\mathbf{F} \\
\mathbf{F}\end{array}$ & & & & 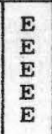 & $\mathrm{E}$ & & & .06 06 & & & & \\
\hline & & 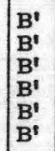 & $\overline{\mathbf{E}}$ & \begin{tabular}{|l}
$G$ \\
$G$ \\
$G$ \\
$G$ \\
$G$ \\
$G$ \\
$G$ \\
$G$
\end{tabular} & $\begin{array}{l}\mathrm{E} \\
\mathrm{E} \\
\mathrm{E} \\
\mathrm{E} \\
\mathrm{E} \\
\mathrm{E}\end{array}$ & 1 & 1 & \begin{tabular}{|l}
$\mathbf{E}$ \\
$\mathbf{E}$ \\
$\mathbf{E}$ \\
$\mathbf{E}$ \\
$\mathbf{E}$ \\
$\mathbf{E}$
\end{tabular} & $\begin{array}{l}\mathbf{F} \\
\mathbf{F} \\
\mathbf{F} \\
\mathbf{F} \\
\mathbf{F} \\
\mathbf{F}\end{array}$ & $\boldsymbol{F}$ & & 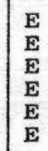 & $\begin{array}{l}F \\
F \\
F \\
F\end{array}$ & $\begin{array}{l}\text { D } \\
\text { D } \\
\text { D } \\
\text { D } \\
\text { D } \\
\text { D }\end{array}$ & $\begin{array}{l}\mathbf{E} \\
\mathbf{E} \\
\mathbf{E} \\
\mathbf{E} \\
\mathbf{E} \\
\mathbf{E}\end{array}$ & $\begin{array}{l}F \\
F \\
F \\
F \\
F \\
F\end{array}$ & $\mid \begin{array}{l}\mathrm{D} \\
\mathrm{D}\end{array}$ & D & $\mid \begin{array}{c}\mathbf{D} \\
\mathrm{B}^{\prime} \\
\mathrm{B}^{\prime} \\
\mathrm{B}^{\prime} \\
\mathbf{B}^{\prime} \\
\mathbf{B}^{\prime}\end{array}$ & 3", & $F$ & $A$ & & & 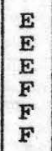 & $\mathbf{E}$ & & & $\begin{array}{l}6 \\
8 \\
6 \\
6\end{array}$ & & & & \\
\hline & & & & \begin{tabular}{|l}
$G$ \\
$G$ \\
$G$ \\
$G$ \\
$G$
\end{tabular} & $\begin{array}{l}\mathbf{E} \\
\mathbf{E} \\
\mathbf{E} \\
\mathbf{E} \\
\mathbf{E}\end{array}$ & \begin{tabular}{|l}
$\mathbf{B}^{\prime}$ \\
$\mathrm{B}^{8}$ \\
$\mathrm{~B}^{\prime}$
\end{tabular} & & \begin{tabular}{|l}
$\mathbf{E}$ \\
$\mathbf{E}$ \\
$\mathbf{E}$ \\
$\mathbf{E}$ \\
$\mathbf{E}$
\end{tabular} & $\begin{array}{l}\mathbf{F} \\
\mathbf{F} \\
\mathbf{F} \\
\mathbf{F} \\
\mathbf{F}\end{array}$ & & & 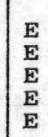 & $\begin{array}{l}\mathbf{F} \\
\mathrm{F}\end{array}$ & & & $\begin{array}{l}F \\
F \\
F \\
F \\
F\end{array}$ & \begin{tabular}{|l}
$\mathrm{E}$ \\
$\mathrm{E}$ \\
$\mathrm{E}$ \\
$\mathrm{D}$ \\
\end{tabular} & : & \begin{tabular}{|l|l}
$\mathbf{B}^{\prime}$ \\
$\mathbf{B}^{\prime}$ \\
$\mathbf{B}^{\prime}$ \\
$\mathbf{B}^{\prime}$ \\
$\mathbf{B}^{\prime}$
\end{tabular} & 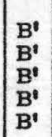 & $\mathbf{F}$ & A & & & $\begin{array}{l}F \\
F \\
F \\
E\end{array}$ & $\begin{array}{l}\mathrm{E} \\
\mathrm{E} \\
\mathrm{F}\end{array}$ & & & & & & & \\
\hline & & & . & \begin{tabular}{|l}
$G$ \\
$G$ \\
$G$ \\
$G$ \\
$G$ \\
$G$
\end{tabular} & & $\begin{array}{l}\mathbf{B}^{\mathbf{B}} \\
\mathbf{B}^{\mathbf{1}} \\
\mathbf{B}^{\mathbf{t}}\end{array}$ & & \begin{tabular}{|l}
$\mathbf{E}$ \\
$\mathbf{E}$ \\
$\mathbf{E}$ \\
$\mathbf{E}$ \\
$\mathbf{E}$
\end{tabular} & $\begin{array}{c}\mathbf{F} \\
\mathbf{F} \\
\mathbf{F} \\
\mathbf{F} \\
\mathbf{F}\end{array}$ & & & & $\begin{array}{l}\underset{F}{F} \\
\vec{F}\end{array}$ & & & 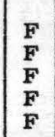 & $\begin{array}{l}\bar{E} \\
D \\
E\end{array}$ & $\begin{array}{l}\mathbf{E} \\
\mathbf{E} \\
\mathbf{E}\end{array}$ & & & $\begin{array}{l}F \\
F \\
F\end{array}$ & & & & $\begin{array}{l}\mathrm{E} \\
\mathrm{E} \\
\mathrm{E} \\
\mathrm{E} \\
\mathrm{E}\end{array}$ & \begin{tabular}{|l}
$E$ \\
$E$ \\
$E$
\end{tabular} & & & & & & & \\
\hline & & $\left|\begin{array}{l}\mathrm{B}^{\prime} \\
\mathrm{B}^{\prime} \\
\mathrm{B}^{\prime}\end{array}\right|$ & 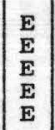 & $\begin{array}{l}G \\
G \\
G \\
G \\
G \\
G\end{array}$ & $\begin{array}{l}\mathrm{E} \\
\mathrm{E} \\
\mathrm{E} \\
\mathrm{E} \\
\mathrm{E}\end{array}$ & $\mid \begin{array}{l}\mathbf{B}^{\mathbf{z}} \\
\mathbf{B}^{\mathbf{B}} \\
\mathbf{A}^{\prime}\end{array}$ & & \begin{tabular}{|l}
$\mathbf{E}$ \\
$\mathbf{E}$ \\
$\mathbf{E}$ \\
$\mathbf{E}$ \\
$\mathbf{E}$
\end{tabular} & F & & & 1 & $\begin{array}{l}\mathbf{F} \\
\mathbf{F} \\
\mathbf{F}\end{array}$ & & & $\begin{array}{l}\mathbf{F} \\
\mathbf{F} \\
\mathbf{F} \\
\mathbf{F}\end{array}$ & $\begin{array}{l}\mathbf{E} \\
\mathbf{E} \\
\mathbf{E}\end{array}$ & $\begin{array}{l}\mathbf{E} \\
\mathbf{E} \\
\mathbf{E} \\
\mathbf{E} \\
\mathbf{E}\end{array}$ & & 1 & 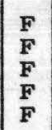 & & & & 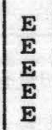 & $\begin{array}{ll}\mathrm{E} \\
\mathrm{I} \\
\mathrm{E}\end{array}$ & & & & & & & \\
\hline & & $\mid \begin{array}{l}B^{\prime} \\
B^{\prime} \\
\mathbf{B}^{\prime}\end{array}$ & $\mid \begin{array}{l}\mathbf{F} \\
\mathbf{E} \\
\mathbf{E}\end{array}$ & N. & $\mathbf{E}$ & \begin{tabular}{|l}
$\mathbf{A}$ \\
$\mathbf{A}$ \\
$\mathbf{A}$
\end{tabular} & & \begin{tabular}{|l}
$\mathbf{E}$ \\
$\mathbf{E}$
\end{tabular} & F & & & & $\begin{array}{l}F \\
F \\
F \\
F\end{array}$ & & $\Xi$ & $\underset{F}{F}$ & $\begin{array}{l}\mathbf{E} \\
\mathbf{E}\end{array}$ & \begin{tabular}{|c|}
$\mathbf{E}$ \\
$\mathbf{E}$ \\
ND \\
$\mathbf{E}$ \\
$\mathbf{E}$
\end{tabular} & & & & & & & $\begin{array}{l}\mathrm{E} \\
\mathrm{E} \\
\mathrm{E}\end{array}$ & $\mathrm{E}$ & & & & & & & \\
\hline & & $\mid \begin{array}{l}\text { A } \\
\text { B } \\
\text { B } \\
\text { B }\end{array}$ & $\mid \begin{array}{l}\mathbf{E} \\
\mathrm{E}\end{array}$ & IN & $\mathbf{F}$ & $\begin{array}{l}\mathrm{A} \\
\mathrm{A}\end{array}$ & 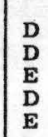 & \begin{tabular}{|l}
$\mathbf{E}$ \\
$\mathbf{E}$ \\
$\mathbf{E}$ \\
$\mathbf{E}$ \\
$\mathbf{E}$
\end{tabular} & $\mathbf{F}$ & & & $\begin{array}{l}E \\
E\end{array}$ & $\begin{array}{l}\text { ND } \\
\text { F } \\
F \\
F \\
F \\
F\end{array}$ & 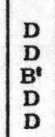 & 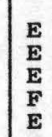 & 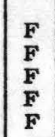 & $\begin{array}{l}\mathrm{D} \\
\mathrm{D}\end{array}$ & $\begin{array}{l}\mathbf{E} \\
\mathbf{E} \\
\mathbf{E} \\
\mathbf{E} \\
\mathbf{N D}\end{array}$ & & $\mathrm{B}^{\circ}$ & $\underset{F}{F}$ & & & & $\begin{array}{l}F \\
F \\
F\end{array}$ & 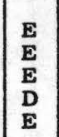 & & & $\begin{array}{c}21 \\
23 \\
09\end{array}$ & & & & \\
\hline & & $\begin{array}{l}\text { B } \\
\text { B } \\
\text { B } \\
\text { B } \\
\text { B }\end{array}$ & \begin{tabular}{|l|l}
$\mathrm{E}$ \\
$\mathrm{E}$ \\
$\mathrm{E}$ \\
$\mathrm{E}$
\end{tabular} & G & $\mathbf{F}$ & $\mathrm{A}_{\mathrm{A}}^{\mathrm{A}}$ & \begin{tabular}{|l}
$E$ \\
$E$ \\
$E$ \\
$D$ \\
$D$
\end{tabular} & $\begin{array}{l}\mathbf{E} \\
\mathbf{E} \\
\mathbf{E}\end{array}$ & 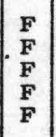 & & $\begin{array}{l}\text { D } \\
\text { D } \\
\text { D }\end{array}$ & & $\vec{F}$ & $\begin{array}{l}\text { D } \\
\text { D } \\
\text { D }\end{array}$ & $E$ & $\begin{array}{l}\mathbf{F} \\
\mathbf{F} \\
\mathbf{F}\end{array}$ & $\begin{array}{l}\mathbf{E} \\
\mathbf{E} \\
\mathbf{E}\end{array}$ & $\begin{array}{l}\text { D } \\
\text { D }\end{array}$ & 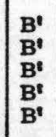 & $\begin{array}{l}\text { D } \\
\mathrm{B}^{\prime} \\
\mathrm{B}^{\prime} \\
\text { D } \\
\mathrm{B}^{\prime}\end{array}$ & $\begin{array}{l}\mathbf{F} \\
\mathbf{F} \\
\mathbf{F}\end{array}$ & & $\begin{array}{l}\mathbf{G} \\
\mathbf{G} \\
\mathbf{G} \\
\mathbf{G} \\
\mathbf{G}\end{array}$ & $\mid \begin{array}{l}\mathbf{B}^{\prime} \\
\mathbf{B}^{\prime} \\
\mathbf{B}^{\prime} \\
\mathbf{B}^{\prime} \\
\mathbf{B}^{\prime}\end{array}$ & $\begin{array}{l}F \\
F \\
F\end{array}$ & $E$ & & & 8 & $\begin{array}{l}\text { G } \\
\text { G } \\
\text { G } \\
\text { G } \\
\text { G }\end{array}$ & & & \\
\hline & $3-\mathrm{R}$ & & & & & & & & & & & & & & & & & & & & & & & & & & & & 07 & & & & \\
\hline
\end{tabular}

lot 1303-spec.

IV - Idaho - 14 


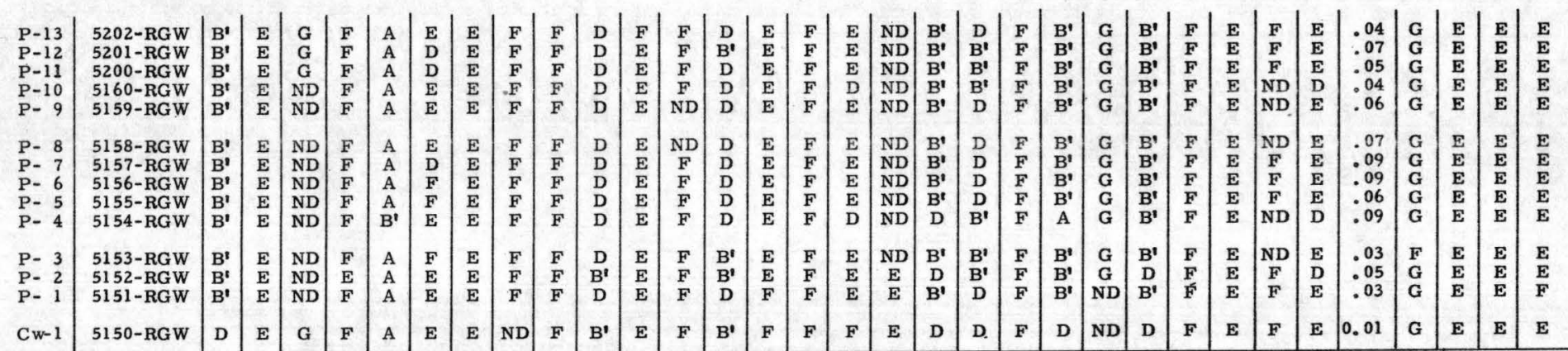


Lone Pine, Idaho, lot 1298

Phosphatic shale member of the Phosphoria formation sampled in bulldozer trench on the west limb of the Snowdrift anticline near Lone Pine Springs, $\mathrm{NE}_{\frac{1}{4}}$ sec. 4, T. $9 \mathrm{~S}_{\circ}$, R. $45 \mathrm{E}_{\circ}$, Caribou County, Idaho. Section measured and sampled by M. A. Warner, R. A. Smart, R. G. Waring, R. S. Jones, and $\mathrm{R}$. P. Sheldon in July 1949 . Samples analyzed for $\mathrm{P}_{2} \mathrm{O}_{5}$ and acid insoluble by U. S. Bureau of

\begin{tabular}{|c|c|c|c|c|c|c|c|c|c|c|c|c|c|c|}
\hline \multirow{2}{*}{$\begin{array}{c}\text { Bed } \\
\text { no. }\end{array}$} & \multirow[b]{2}{*}{ Rock description } & \multirow{2}{*}{$\begin{array}{c}\text { Sample } \\
\text { no. }\end{array}$} & \multirow{2}{*}{$\begin{array}{c}\text { Thickness } \\
\text { (feet) }\end{array}$} & \multicolumn{5}{|c|}{ Chemical analyses (percent) } & \multirow{2}{*}{$\begin{array}{c}\text { Cumulative } \\
\text { thickness } \\
\text { (feet) }\end{array}$} & \multirow{2}{*}{$\begin{array}{l}\text { Thickness } \mathrm{x} \\
\text { percent } \mathrm{P}_{2} \mathrm{O}_{5} \\
\text { (cumulative) }\end{array}$} & \multirow{2}{*}{\multicolumn{2}{|c|}{$\begin{array}{c}\text { Uranium content } \\
\text { (percent) }\end{array}$}} & \multirow{2}{*}{$\begin{array}{l}\text { Thickness } x \\
\text { percent eU } \\
\text { (cumulative) }\end{array}$} & \\
\hline & & & & $\mathrm{P}_{2} \mathrm{O}_{5}$ & $\mathrm{Al}_{2} \mathrm{O}_{3}$ & $\mathrm{Fe}_{2} \mathrm{O}_{3}$ & $\begin{array}{l}\text { Loss on } \\
\text { ignition }\end{array}$ & $\begin{array}{c}\text { Acid } \\
\text { insoluble }\end{array}$ & & & & & & \\
\hline \multicolumn{14}{|c|}{ Rex chert member of Phosphoria formation - basal bed only } & \\
\hline $\mathrm{R}-11$ & Chert & -- & 1.8 & $\cdots$ & $\cdots$ & $-\cdot$ & $-\cdot$ & -- & 1.8 & -- & -- & -- & -- & \\
\hline \multicolumn{14}{|c|}{ Phosphatic shale member of Phosphoria formation } & \\
\hline $\begin{array}{l}P-115 \\
P-114 \\
P-113 \\
P-112 \\
P-111\end{array}$ & $\begin{array}{l}\text { Mudstone } \\
\text { Phosphate rock, argillaceous } \\
\text { Mudstone } \\
\text { Mudstone } \\
\text { Mudstone }\end{array}$ & $\begin{array}{l}\text { 5080-MAW } \\
\text { 5006-MAW } \\
\text { 5007-MAW } \\
\text { 5008-MAW } \\
\text { 5009-MAW }\end{array}$ & $\begin{array}{r}0.9 \\
.5 \\
.7 \\
.9 \\
2.0\end{array}$ & $\begin{array}{r}2.1 \\
29.6 \\
3.6 \\
2.8 \\
2.0\end{array}$ & $\begin{array}{l}\because- \\
\because- \\
\because-\end{array}$ & $\begin{array}{l}-- \\
\therefore \\
\therefore \\
--\end{array}$ & $\begin{array}{l}\because- \\
\therefore \\
--\end{array}$ & $\begin{array}{l}84.4 \\
17.1 \\
73.8 \\
69.7 \\
75.0\end{array}$ & $\begin{array}{l}0.9 \\
1.4 \\
2.1 \\
3.0 \\
5.0\end{array}$ & $\begin{array}{r}1.89 \\
16.69 \\
19.21 \\
21.73 \\
25.73\end{array}$ & $\begin{array}{l}0.002 \\
.013 \\
.004 \\
.003 \\
.003\end{array}$ & $\begin{array}{c}-\overline{012} \\
0 . \\
\cdots\end{array}$ & $\begin{array}{l}0.002 \\
.008 \\
.011 \\
.014 \\
.020\end{array}$ & \\
\hline $\begin{array}{l}P-110 \\
P-109 \\
P-108 \\
P-107 \\
P-106\end{array}$ & $\begin{array}{l}\text { Mudstone } \\
\text { Mudstone } \\
\text { Mudstone } \\
\text { Mudstane } \\
\text { Phosphate rock, argillaceous }\end{array}$ & $\begin{array}{l}\text { 5010-MAW } \\
5011-\mathrm{MAW} \\
\text { 5012-MAW } \\
\text { 5013-MAW } \\
5014-\mathrm{MAW}\end{array}$ & $\begin{array}{l}2.3 \\
3.2 \\
1.1 \\
3.3 \\
1.0\end{array}$ & $\begin{array}{r}1.9 \\
.9 \\
3.7 \\
27.8\end{array}$ & $\begin{array}{l}\because- \\
\because- \\
2.8\end{array}$ & $\begin{array}{l}-. \\
\because- \\
1.24\end{array}$ & $\begin{array}{l}-- \\
\because- \\
-- \\
4.08\end{array}$ & $\begin{array}{l}79.0 \\
79.2 \\
77.7 \\
70.3 \\
21.1\end{array}$ & $\begin{array}{l}7.3 \\
10.5 \\
11.6 \\
14.9 \\
15.9\end{array}$ & $\begin{array}{l}27.80 \\
31.00 \\
31.99 \\
44.20 \\
72.00\end{array}$ & $\begin{array}{l}.003 \\
.003 \\
.002 \\
.004 \\
.010\end{array}$ & $\begin{array}{l}-- \\
\ddot{0} \\
--\end{array}$ & $\begin{array}{l}.027 \\
.036 \\
.038 \\
.052 \\
.062\end{array}$ & $\mathbb{N}$ \\
\hline $\begin{array}{l}P-105 \\
P-104 \\
P-103 \\
P-102 \\
P-101\end{array}$ & $\begin{array}{l}\text { Mudstone } \\
\text { Phosphate rock } \\
\text { Phosphate rock } \\
\text { Phosphate rock } \\
\text { Mudstone }\end{array}$ & $\begin{array}{l}\text { 5015-MAW } \\
\text { 5016-MAW } \\
\text { 5017-MAW } \\
\text { 5018-MAW } \\
\text { 5019-MAW }\end{array}$ & $\begin{array}{r}2.2 \\
1.5 \\
.9 \\
1.0 \\
1.5\end{array}$ & $\begin{array}{r}3.8 \\
36.9 \\
35.7 \\
36.5 \\
6.6\end{array}$ & $\begin{array}{l}9.5 \\
1.2 \\
1.5 \\
1.2 \\
8.8\end{array}$ & $\begin{array}{r}3.34 \\
.82 \\
.67 \\
.50 \\
3.23\end{array}$ & $\begin{array}{l}5.88 \\
4.08 \\
5.96 \\
2.56 \\
4.94\end{array}$ & $\begin{array}{r}71.7 \\
2.8 \\
2.8 \\
4.9 \\
66.7\end{array}$ & $\begin{array}{l}18.1 \\
19.6 \\
20.5 \\
21.5 \\
23.0\end{array}$ & $\begin{array}{r}80.36 \\
135.71 \\
167.84 \\
204.34 \\
214.24\end{array}$ & $\begin{array}{l}.003 \\
.011 \\
.013 \\
.012 \\
.004\end{array}$ & $\begin{array}{l}.0 \\
.010 \\
.011 \\
.011 \\
--\end{array}$ & $\begin{array}{l}.068 \\
.085 \\
.096 \\
.108 \\
.114\end{array}$ & $\frac{b}{b}$ \\
\hline $\begin{array}{l}P-100 \\
P-99 \\
P-98 \\
P-97 \\
P-96\end{array}$ & $\begin{array}{l}\text { Phosphate rock and argillaceous } \\
\text { phosphate rock } \\
\text { Phosphate rock, argillaceous } \\
\text { Phosphate rock-. } \\
\text { Phosphate rock } \\
\text { Mudstone }\end{array}$ & $\begin{array}{l}\text { 5081-MAW } \\
5082-\mathrm{MAW} \\
5083-\mathrm{MAW} \\
5084-\mathrm{MAW} \\
5085-\mathrm{MAW}\end{array}$ & $\begin{array}{r}.7 \\
.4 \\
.9 \\
1.0 \\
.6\end{array}$ & $\begin{array}{r}29.6 \\
18.3 \\
31.7 \\
34.1 \\
7.1\end{array}$ & $\begin{array}{l}3.2 \\
6.0 \\
2.5 \\
1.5 \\
8.5\end{array}$ & $\begin{array}{l}1.32 \\
2.10 \\
1.07 \\
.59 \\
3.29\end{array}$ & $\begin{array}{l}3.56 \\
4.84 \\
3.36 \\
3.22 \\
8.32\end{array}$ & $\begin{array}{r}18.0 \\
41.8 \\
13.4 \\
9.6 \\
63.7\end{array}$ & $\begin{array}{l}23.7 \\
24.1 \\
25.0 \\
26.0 \\
26.6\end{array}$ & $\begin{array}{l}234.96 \\
242.28 \\
270.81 \\
304.91 \\
309.17\end{array}$ & $\begin{array}{l}.010 \\
.005 \\
.013 \\
.013 \\
.005\end{array}$ & $\begin{array}{l}.010 \\
.005 \\
.012 \\
.012 \\
.004\end{array}$ & $\begin{array}{l}.122 \\
.124 \\
.135 \\
.148 \\
.151\end{array}$ & $\sum$ \\
\hline $\begin{array}{l}\text { P- } 95 \\
\text { P- } 94 \\
\text { P- } 93 \\
\text { P- } 92 \\
\text { P- } 91\end{array}$ & $\begin{array}{l}\text { Phosphate rock } \\
\text { Mudstone, phosphatic } \\
\text { Phosphate rock- } \\
\text { Phosphate rock, argillaceous } \\
\text { Phosphate rock- }\end{array}$ & $\begin{array}{l}\text { 5086-MAW } \\
\text { 5087-MAW } \\
\text { 5088-MAW } \\
\text { 5089-MAW } \\
\text { 5090-MAW }\end{array}$ & $\begin{array}{r}1.2 \\
.7 \\
.6 \\
.5 \\
1.2\end{array}$ & $\begin{array}{l}36.2 \\
14.3 \\
34.6 \\
20.3 \\
35.8\end{array}$ & $\begin{array}{l}.78 \\
6.7 \\
1.4 \\
5.6 \\
1.1\end{array}$ & $\begin{array}{r}.23 \\
2.80 \\
.38 \\
1.30 \\
.72\end{array}$ & $\begin{array}{r}3.08 \\
8.20 \\
4.26 \\
10.06 \\
2.76\end{array}$ & $\begin{array}{r}6.4 \\
47.9 \\
8.0 \\
30.3 \\
6.0\end{array}$ & $\begin{array}{l}27.8 \\
28.5 \\
29.1 \\
29.6 \\
30.8\end{array}$ & $\begin{array}{l}352.61 \\
362.62 \\
383.38 \\
393.53 \\
436.49\end{array}$ & $\begin{array}{l}.019 \\
.006 \\
.015 \\
.013 \\
.017\end{array}$ & $\begin{array}{l}.018 \\
.005 \\
.013 \\
.012 \\
.017\end{array}$ & $\begin{array}{l}.174 \\
.178 \\
.187 \\
.194 \\
.214\end{array}$ & \\
\hline $\begin{array}{l}\text { P- } 90 \\
\text { P- } 89 \\
P-88\end{array}$ & $\begin{array}{l}\text { Mudstone, phosphatic } \\
\text { Phosphate rock } \\
\text { Phosphate rock }\end{array}$ & $\begin{array}{l}5091-\text { MAW } \\
5020-\text { RAS } \\
5021-\text { RAS }\end{array}$ & $\begin{array}{r}1.3 \\
1.0 \\
.9\end{array}$ & $\begin{array}{l}11.7 \\
31.9 \\
38.5\end{array}$ & $\begin{array}{l}6.8 \\
2.0 \\
.77\end{array}$ & $\begin{array}{r}2.84 \\
.76 \\
.44\end{array}$ & $\begin{array}{l}5.34 \\
1.84 \\
2.70\end{array}$ & $\begin{array}{r}55.7 \\
13.5 \\
1.2\end{array}$ & $\begin{array}{l}32.1 \\
33.1 \\
34.0\end{array}$ & $\begin{array}{l}451.70 \\
483.60 \\
518.25\end{array}$ & $\begin{array}{l}.004 \\
.015 \\
.016\end{array}$ & $\begin{array}{l}. \overline{-} \\
.010 \\
.016\end{array}$ & $\begin{array}{l}.219 \\
.234 \\
.249\end{array}$ & \\
\hline
\end{tabular}

mples analyzed for eU and chem. U by the U. S. Geological Surve laboratory, Geochemistry and 


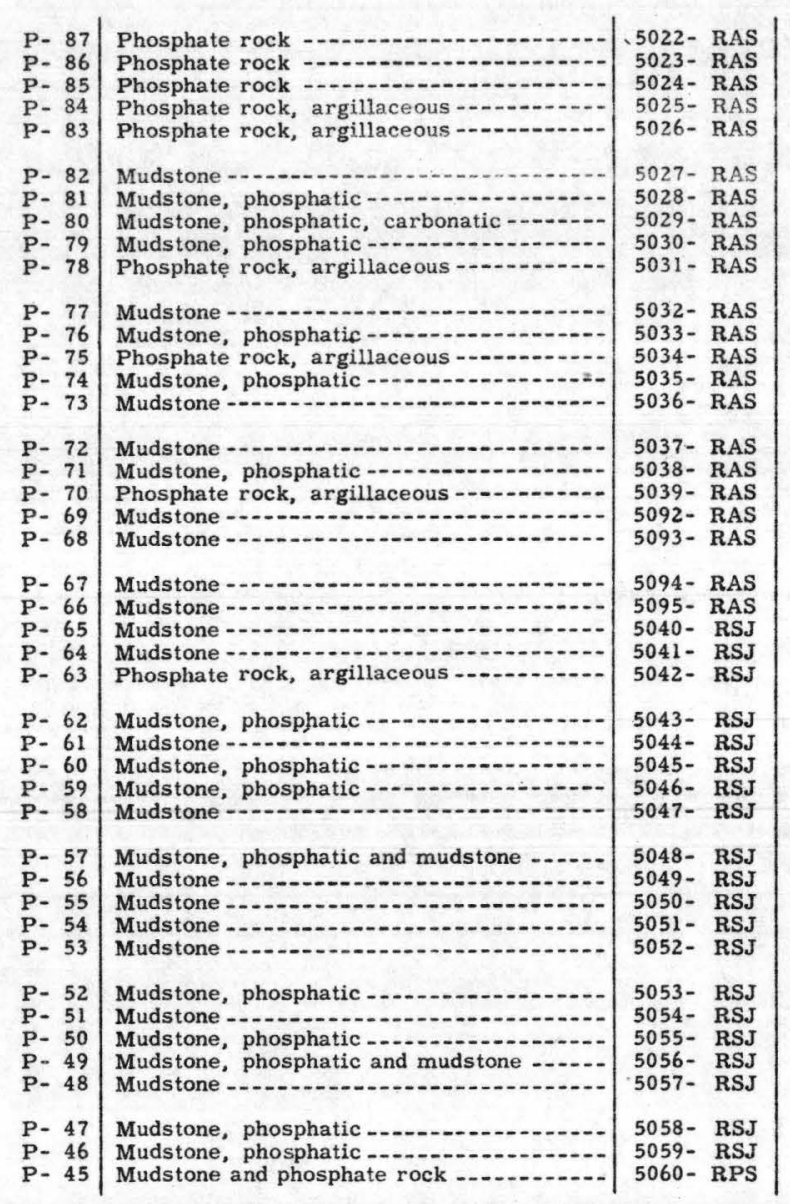

\begin{tabular}{|c|c|c|c|c|}
\hline 1.5 & 33.5 & 2.3 & 1.11 & 3.92 \\
\hline 1.2 & 27.4 & 2. 9 & 1.96 & 9.82 \\
\hline 1. & $\begin{array}{r}32.5 \\
\end{array}$ & 1.7 & .51 & 8.54 \\
\hline 2.2 & $\begin{array}{r}25.8 \\
22.6\end{array}$ & $\begin{array}{r}3.4 \\
4.0\end{array}$ & $\begin{array}{l}1.24 \\
1.34\end{array}$ & $\begin{array}{l}13.56 \\
14.36\end{array}$ \\
\hline 2.3 & 65 & $\ldots$ & $\ldots$ & nes \\
\hline 2.5 & 15.4 & -. & -. & -. \\
\hline .0 & 10.7 & -- & -- & -- \\
\hline & 12.8 & -- & -- & -- \\
\hline 1.3 & 24.2 & -- & - & $\cdots$ \\
\hline .5 & 4.5 & -- & -- & . \\
\hline 1.0 & 10.1 & -- & $\cdots$ & -- \\
\hline & 20.7 & -- & - & -- \\
\hline .8 & 12.2 & -- & $\overline{-}$ & $\bar{*}$ \\
\hline 1.4 & 64 & $\ldots$ & $\ldots$ & $\ldots$ \\
\hline .4 & 8.6 & -. & -. & -- \\
\hline 5 & 18.4 & -- & -. & -- \\
\hline 4 & 5.6 & -- & - & $\cdots$ \\
\hline .2 & 2.7 & & $\cdots$ & $\cdots$ \\
\hline .0 & 3. 0 & -- & -- & $\cdots$ \\
\hline 3 & 4.2 & -- & -- & -- \\
\hline .0 & 2.8 & $=-$ & 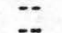 & $=$ \\
\hline .2 & 24.5 & -- & -- & -- \\
\hline .7 & 14.3 & -- & -- & -- \\
\hline & 7.5 & -- & - & -- \\
\hline .8 & 14.4 & $\overline{-}$ & $=$ & $\overline{-}$ \\
\hline .7 & 3.1 & - & -- & -. \\
\hline .7 & 3,0 & -. & - & -- \\
\hline & & 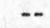 & 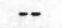 & \\
\hline & 7.5 & - & 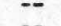 & - \\
\hline 0 & $\begin{array}{l}1.2 \\
.6\end{array}$ & - & -. & $\ldots$ \\
\hline 6 & 10.1 & -. & $\ldots$ & $\ldots$ \\
\hline .5 & .4 & -- & -- & -- \\
\hline & 18.2 & - & $\cdots$ & -- \\
\hline 5 & 8.6 & -- & -- & -- \\
\hline & & & & \\
\hline & $\begin{array}{l}14.4 \\
13.0\end{array}$ & - & 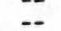 & - \\
\hline & 15.9 & -- & -- & -- \\
\hline
\end{tabular}

8.7
14.8
6.3
16.1
22.2
61.9
38.0
37.9
51.0
29.5
73.0
61.7
34.1
54.2
66.8
67.3
64.2
40.2
67.3
76.5
67.1
70.4
75.0
72.3
23.5
61.0
66.0
38.0
58.5
76.5
83.0
83.6
69.8
87.2
85.9
64.7
90.2
45.8
64.2
72.8
38.3
42.8
40.4

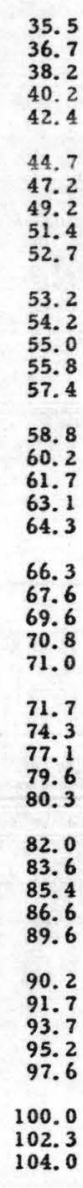

568.50
601.38
650.13
701.73
751.45
766.40
804.90
82.30
85.30
885.46
85.92
888.17
898.27
914.83
924.59
936.59
945.55
957.59
985.19
993.03
996.27
$1,002.27$
$1,007.73$
$1,013.33$
$1,017.53$
$1,022.43$
$1,032.44$
$1,051.94$
$1,092.26$
$1,118.51$
$1,120.68$
$1,125.78$
$1,126.90$
$1,140.40$
$1,141.84$
$1,143.64$
$1,149.70$
$1,150.30$
$1,186.70$
$1,199.60$
$1,210.16$
$1,244.72$
$1,274.62$
$1,301.65$

\begin{tabular}{|c|c|c|}
\hline $\begin{array}{l}.016 \\
.016 \\
.015 \\
.010 \\
.009\end{array}$ & $\begin{array}{l}.014 \\
.014 \\
.015 \\
.009 \\
.008\end{array}$ & $\begin{array}{l}.273 \\
.292 \\
.314 \\
.334 \\
.354\end{array}$ \\
\hline $\begin{array}{l}.004 \\
.003 \\
.005 \\
.005 \\
.005\end{array}$ & $\begin{array}{l}.002 \\
.003 \\
.0003 \\
.-\end{array}$ & $\begin{array}{l}.363 \\
.371 \\
.381 \\
.392 \\
.398\end{array}$ \\
\hline $\begin{array}{l}.002 \\
.004 \\
.007 \\
.004 \\
.003\end{array}$ & $\begin{array}{c}- \\
-\overline{-0} \\
.0 \\
\because-\end{array}$ & $\begin{array}{l}.399 \\
.403 \\
.409 \\
.412 \\
.417\end{array}$ \\
\hline $\begin{array}{l}.003 \\
.004 \\
.005 \\
.004 \\
.003\end{array}$ & $\begin{array}{c}\ddot{-} \\
.005 \\
\because-\end{array}$ & $\begin{array}{l}.421 \\
.427 \\
.434 \\
.440 \\
.444\end{array}$ \\
\hline $\begin{array}{l}.004 \\
.003 \\
.004 \\
.002 \\
.007\end{array}$ & $\begin{array}{c}z \\
\because \\
\ddot{0005}\end{array}$ & $\begin{array}{l}.452 \\
.455 \\
.463 \\
.466 \\
.467\end{array}$ \\
\hline $\begin{array}{l}.005 \\
.003 \\
.006 \\
.004 \\
.002\end{array}$ & \begin{tabular}{l}
.003 \\
$.0-04$ \\
-004 \\
\hdashline-
\end{tabular} & $\begin{array}{l}.471 \\
.478 \\
.495 \\
.505 \\
.507\end{array}$ \\
\hline $\begin{array}{l}.003 \\
.002 \\
.004 \\
.003 \\
.003\end{array}$ & -: & $\begin{array}{l}.512 \\
.515 \\
.522 \\
.526 \\
.535\end{array}$ \\
\hline $\begin{array}{l}.004 \\
.002 \\
.004 \\
.004 \\
.004\end{array}$ & -- & $\begin{array}{l}.537 \\
.540 \\
.548 \\
.554 \\
.564\end{array}$ \\
\hline $\begin{array}{l}.008 \\
.006 \\
.005\end{array}$ & $\begin{array}{l}.006 \\
.004 \\
.004\end{array}$ & $\begin{array}{r}.583 \\
.597 \\
.605\end{array}$ \\
\hline
\end{tabular}


$\underline{\text { Lone Pine-Continued }}$

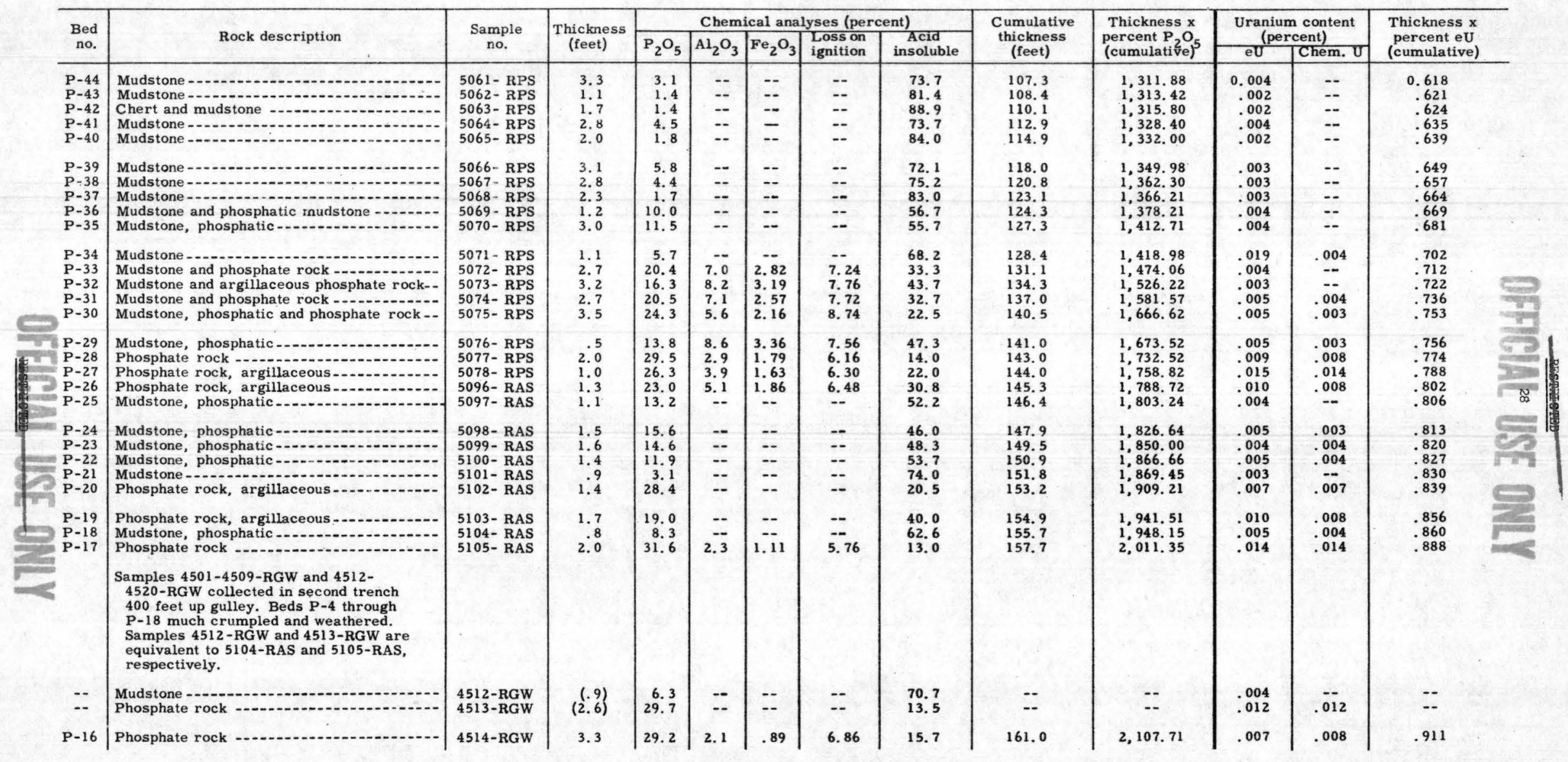




\begin{tabular}{|c|c|c|c|c|c|c|c|c|c|c|c|c|c|}
\hline $\begin{array}{l}P-15 \\
P-14 \\
P-13 \\
P-12 \\
P-11\end{array}$ & $\begin{array}{l}\text { Phosphate rock } \\
\text { Phosphate rock } \\
\text { Mudstone, phosphatic } \\
\text { Phosphate rock } \\
\text { Phosphate rock, argillaceous }\end{array}$ & $\begin{array}{l}\text { 4515-RGW } \\
4516-\mathrm{RGW} \\
4517-\mathrm{RGW} \\
4518-\mathrm{RGW} \\
4519-\mathrm{RGW}\end{array}$ & $\begin{array}{l}2.8 \\
1.6 \\
.7 \\
.8 \\
.9\end{array}$ & $\begin{array}{l}31.5 \\
32.5 \\
14.1 \\
31.6 \\
24.6\end{array}$ & $\begin{array}{l}1.7 \\
2.0 \\
8.2 \\
1.8 \\
4.8\end{array}$ & $\begin{array}{r}.80 \\
.83 \\
3.01 \\
1.10 \\
2.26\end{array}$ & $\begin{array}{l}7.06 \\
7.84 \\
8.18 \\
6.82 \\
6.80\end{array}$ & $\begin{array}{r}10.1 \\
6.7 \\
44.1 \\
9.6 \\
23.4\end{array}$ & $\begin{array}{l}163.8 \\
165.4 \\
166.1 \\
166.9 \\
167.8\end{array}$ & $\begin{array}{l}2,195.91 \\
2,247.91 \\
2,257.78 \\
2,283.06 \\
2,305.20\end{array}$ & $\begin{array}{l}.012 \\
.012 \\
.006 \\
.005 \\
.004\end{array}$ & $\begin{array}{l}.013 \\
.011 \\
.004 \\
.004 \\
-.\end{array}$ & $\begin{array}{l}.945 \\
.964 \\
.968 \\
.972 \\
.976\end{array}$ \\
\hline $\begin{array}{l}\text { P-10 } \\
\text { P- } 9 \\
\text { P- } 8 \\
\text { P- } 7 \\
\text { P- } 6\end{array}$ & $\begin{array}{l}\text { Phosphate rock } \\
\text { Phosphate rock } \\
\text { Phosphate rock } \\
\text { Phosphate rock } \\
\text { Phosphate rock }\end{array}$ & $\begin{array}{l}4520-R G W \\
4501-R G W \\
4502-R G W \\
4503-R G W \\
4504-R G W\end{array}$ & $\begin{array}{l}2.0 \\
2.0 \\
1.0 \\
1.2 \\
.9\end{array}$ & $\begin{array}{l}35.6 \\
35.3 \\
31.6 \\
33.6 \\
31.7\end{array}$ & $\begin{array}{l}.94 \\
.77 \\
1.3 \\
.74 \\
1.1\end{array}$ & $\begin{array}{l}.40 \\
.37 \\
.58 \\
.44 \\
.68\end{array}$ & $\begin{array}{l}6.20 \\
6.94 \\
8.60 \\
6.50 \\
7.48\end{array}$ & $\begin{array}{l}1.5 \\
1.0 \\
5.9 \\
3.0 \\
5.6\end{array}$ & $\begin{array}{l}169.8 \\
171.8 \\
172.8 \\
174.0 \\
174.9\end{array}$ & $\begin{array}{l}2,376.40 \\
2,447.00 \\
2,478.60 \\
2,518.92 \\
2,547.45\end{array}$ & $\begin{array}{l}.008 \\
.007 \\
.011 \\
.023 \\
.027\end{array}$ & $\begin{array}{l}.006 \\
.006 \\
.010 \\
.021 \\
.026\end{array}$ & $\begin{array}{l}.992 \\
1.006 \\
1.017 \\
1.045 \\
1.069\end{array}$ \\
\hline $\begin{array}{l}\text { P- } 5 \\
\text { P- } 4 \\
\text { P- } 3 \\
\text { P- } 2 \\
\text { P- } 1\end{array}$ & $\begin{array}{l}\text { Phosphate rock } \\
\text { Mudstone } \\
\text { Carbonate rock, argillaceous } \\
\text { Mudstone, carbonatic } \\
\text { Phosphate rock ........ }\end{array}$ & $\begin{array}{l}\text { 4505-RGW } \\
4506-\mathrm{RGW} \\
4507-\mathrm{RGW} \\
4508-\mathrm{RGW} \\
4509-\mathrm{RGW}\end{array}$ & $\begin{array}{r}.8 \\
1.9 \\
2.7 \\
2.0 \\
.5\end{array}$ & $\begin{array}{r}32.7 \\
2.8 \\
.3 \\
1.1 \\
34.4\end{array}$ & \begin{tabular}{l}
1.1 \\
\hdashline- \\
\hdashline- \\
--
\end{tabular} & \begin{tabular}{l}
.46 \\
\hdashline- \\
\hdashline- \\
--
\end{tabular} & $\begin{array}{l}5.68 \\
=- \\
\because- \\
--\end{array}$ & $\begin{array}{r}7.2 \\
65.2 \\
33.9 \\
63.8 \\
5.0\end{array}$ & $\begin{array}{l}175.7 \\
177.6 \\
180.3 \\
182.3 \\
182.8\end{array}$ & $\begin{array}{l}2,573.61 \\
2,578.93 \\
2,579.74 \\
2,581.94 \\
2,599.14\end{array}$ & $\begin{array}{l}.010 \\
.005 \\
.001 \\
.003 \\
.011\end{array}$ & $\begin{array}{l}.009 \\
.003 \\
0- \\
.011\end{array}$ & $\begin{array}{l}1.077 \\
1.086 \\
1.089 \\
1.095 \\
1.101\end{array}$ \\
\hline \multicolumn{14}{|c|}{ Wells formation - top bed only } \\
\hline$C w-1$ & Carbonate rock & -. & 1.1 & 0.8 & -. & -- & -- & 3.6 & 1.1 & -- & -- & -- & -- \\
\hline
\end{tabular}


Georgetown Canyon, Idaho, lot 1320

Portion of the phosphatic shale member of the Phosphoria formation sampled from diamond drill hole on east limb of the Geortetown

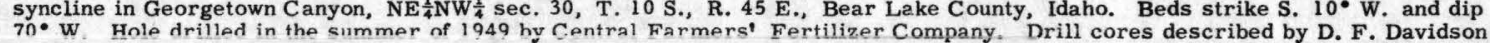
Drill cores described by D. F. in September 1949. Samples analyzed by U. S. Bureau of Mines laboratory, Albany, Oreg.

\begin{tabular}{|c|c|c|c|c|c|c|c|c|c|c|}
\hline \multirow{2}{*}{$\begin{array}{l}\text { Bed } \\
\text { no. }\end{array}$} & \multirow[t]{2}{*}{ Rock description } & \multirow{2}{*}{$\begin{array}{c}\text { Sample } \\
\text { no. }\end{array}$} & \multirow{2}{*}{$\begin{array}{l}\text { Thickness } \\
\text { (feet) }\end{array}$} & \multicolumn{2}{|c|}{$\begin{array}{c}\text { Chemical analyses } \\
\text { (percent) }\end{array}$} & \multirow{2}{*}{$\begin{array}{c}\text { Cumulative } \\
\text { thickness } \\
\text { (feet) }\end{array}$} & \multirow{2}{*}{$\begin{array}{l}\text { Thickness } x \\
\text { percent } \mathrm{P}_{2} \mathrm{O}_{5-} \\
\text { (cumulative) }^{-}\end{array}$} & \multicolumn{2}{|c|}{$\begin{array}{l}\text { Uranium content } \\
\text { (percent) }\end{array}$} & \multirow{2}{*}{$\begin{array}{c}\text { Thickness } x \\
\text { percent chem. } \\
\text { (cumulative) }\end{array}$} \\
\hline & & & & $\mathrm{P}_{2} \mathrm{O}_{5}$ & insoluble & & & $\mathrm{eU}$ & Chem. U & \\
\hline \multicolumn{6}{|c|}{ Phosphatic shale member of Phosphoria formation } & * & & & & \\
\hline $\begin{array}{l}P-15 \\
P-14 \\
P-13 \\
P-12 \\
P-11\end{array}$ & $\begin{array}{l}\text { Mudstone } \\
\text { Mudstone, phosphatic, carbonatic } \\
\text { Mudstone, carbonatic } \\
\text { Carbonate rock, argilaceous } \\
\text { Mudstone, phosphatic, carbonatic }\end{array}$ & $\begin{array}{l}\text { 4616-DFD } \\
4617-D F D \\
4618-D F D \\
4619-D F D \\
4620-D F D\end{array}$ & $\begin{array}{l}2.0 \\
.6 \\
1.3 \\
1.2 \\
1.3\end{array}$ & $\begin{array}{r}4.0 \\
9.5 \\
5.1 \\
.9 \\
8.9\end{array}$ & $\begin{array}{l}68.9 \\
46.1 \\
55.5 \\
32.9 \\
45.3\end{array}$ & $\begin{array}{l}2.0 \\
2.6 \\
3.9 \\
5.1 \\
6.4\end{array}$ & $\begin{array}{r}8.00 \\
13.70 \\
20.33 \\
21.41 \\
32.98\end{array}$ & $\begin{array}{l}0.003 \\
.003 \\
.003 \\
.001 \\
.004\end{array}$ & $\begin{array}{l}0.002 \\
.005 \\
.003 \\
.001 \\
.005\end{array}$ & $\begin{array}{l}0.004 \\
.007 \\
.011 \\
.012 \\
.019\end{array}$ \\
\hline $\begin{array}{l}\text { P-10 } \\
\text { P- } 9 \\
\text { P- } 8 \\
\text { P- } 7 \\
\text { P- } 6\end{array}$ & $\begin{array}{l}\text { Mudstone, carbonatic } \\
\text { Mudstone } \\
\text { Carbonate rock, argillaceous } \\
\text { Carbonate rock, argillaceous } \\
\text { Carbonate rock, argillaceous }\end{array}$ & $\begin{array}{l}4621-\text { DFD } \\
4622-\text { DFD } \\
4623-\text { DFD } \\
4624-\text { DFD } \\
4625-\text { DFD }\end{array}$ & $\begin{array}{l}2.5 \\
2.5 \\
5.5 \\
3.5 \\
.7\end{array}$ & $\begin{array}{r}2.5 \\
2.5 \\
1.9 \\
.2 \\
.3\end{array}$ & $\begin{array}{l}57.1 \\
66.0 \\
29.0 \\
17.9 \\
29.9\end{array}$ & $\begin{array}{r}8.9 \\
11.4 \\
16.9 \\
20.4 \\
21.1\end{array}$ & $\begin{array}{l}39.23 \\
45.48 \\
55.93 \\
56.63 \\
56.84\end{array}$ & $\begin{array}{l}.002 \\
.003 \\
.001 \\
.001 \\
.001\end{array}$ & $\begin{array}{l}.002 \\
.002 \\
.001 \\
.001 \\
.001\end{array}$ & $\begin{array}{l}.024 \\
.029 \\
.034 \\
.038 \\
.038\end{array}$ \\
\hline $\begin{array}{l}\text { P- } 5 \\
\text { P- } 4 \\
\text { P- } 3 \\
\text { P- } 2 \\
\text { P- } 1\end{array}$ & $\begin{array}{l}\text { Carbonate rock, argillace ous } \\
\text { Mudstone, carbonatic } \\
\text { Carbonate rock, argillaceous } \\
\text { Mudstone } \\
\text { Mudstone }\end{array}$ & $\begin{array}{l}4626-\text { DFD } \\
4627-\text { DFD } \\
4628-D F D \\
4629-\text { DFD } \\
4630-\text { DFD }\end{array}$ & $\begin{array}{l}3.0 \\
5.0 \\
5.0 \\
2.5 \\
2.0\end{array}$ & $\begin{array}{r}.2 \\
1.4 \\
7.1 \\
22.4 \\
17.5\end{array}$ & $\begin{array}{l}30.4 \\
62.0 \\
20.7 \\
27.3 \\
31.8\end{array}$ & $\begin{array}{l}24.1 \\
29.1 \\
34.1 \\
36.6 \\
38.6\end{array}$ & $\begin{array}{r}57.44 \\
64.44 \\
99.94 \\
155.94 \\
190.94\end{array}$ & $\begin{array}{l}.001 \\
.002 \\
.003 \\
.009 \\
.005\end{array}$ & $\begin{array}{l}.001 \\
.002 \\
.003 \\
.010 \\
.006\end{array}$ & $\begin{array}{l}.041 \\
.051 \\
.066 \\
.091 \\
.103\end{array}$ \\
\hline
\end{tabular}


Dingle, Idaho, lot 1293

Phosphatic shale member of the Phosphoria formation sampled in bulldozer trench on the east limb of the Hot Springs anticline, SE $\frac{1}{4}$ sec. $36, T .14 \mathrm{~S}$., R. P. Sheldon and sampled by Warner, Smart, and Waring in July 1949 . Samples analyzed for $\mathrm{P}_{2} \mathrm{O}_{5}$ and acid insoluble by $U$. $S$. Bureau of Mines laboratory, Albany, Oreg., and for other constituents by Trace Elements Section laboratory, $\mathrm{U}_{2} \mathrm{~S}_{5}$. Geological Survey, Washington, $\mathrm{D}$. C.

Samples analyzed for eU and chem. U by the U. S. Geological Surve laboratory, Geochemistry and Petrology Branch.

\begin{tabular}{|c|c|c|c|c|c|c|c|c|c|c|c|c|}
\hline \multirow{2}{*}{$\begin{array}{c}\text { Bed } \\
\text { no. }\end{array}$} & \multirow{2}{*}{ Rock description } & \multirow{2}{*}{$\begin{array}{l}\text { Sample } \\
\text { no. }\end{array}$} & \multirow{2}{*}{$\mid \begin{array}{c}\text { Thickness } \\
\text { (feet) }\end{array}$} & \multicolumn{5}{|c|}{ Chemical analyses (percent) } & \multirow{2}{*}{$\begin{array}{l}\text { Cumulative } \\
\text { thickness } \\
\text { (feet) }\end{array}$} & \multirow{2}{*}{$\begin{array}{l}\text { Thickness } x \\
\text { percent } P_{2} O_{5} \\
\text { (cumulative) }\end{array}$} & \multirow{2}{*}{$\begin{array}{c}\begin{array}{c}\text { Uranium content } \\
\text { (percent) }\end{array} \\
\begin{array}{c|c|c|}\text { eU } & \text { Chem. U }\end{array}\end{array}$} & \multirow{2}{*}{$\begin{array}{l}\text { Thickness } x \\
\text { percent eU } \\
\text { (cumulative) }\end{array}$} \\
\hline & & & & $\mathrm{P}_{2} \mathrm{O}_{5}$ & $\mathrm{Al}_{2} \mathrm{O}_{3}$ & $\mathrm{Fe}_{2} \mathrm{O}_{3}$ & $\begin{array}{l}\text { Loss on } \\
\text { ignition }\end{array}$ & $\begin{array}{c}\text { Acid } \\
\text { insoluble }\end{array}$ & & & & \\
\hline
\end{tabular}

Rex chert member of Phosphoria formation - basal beds only

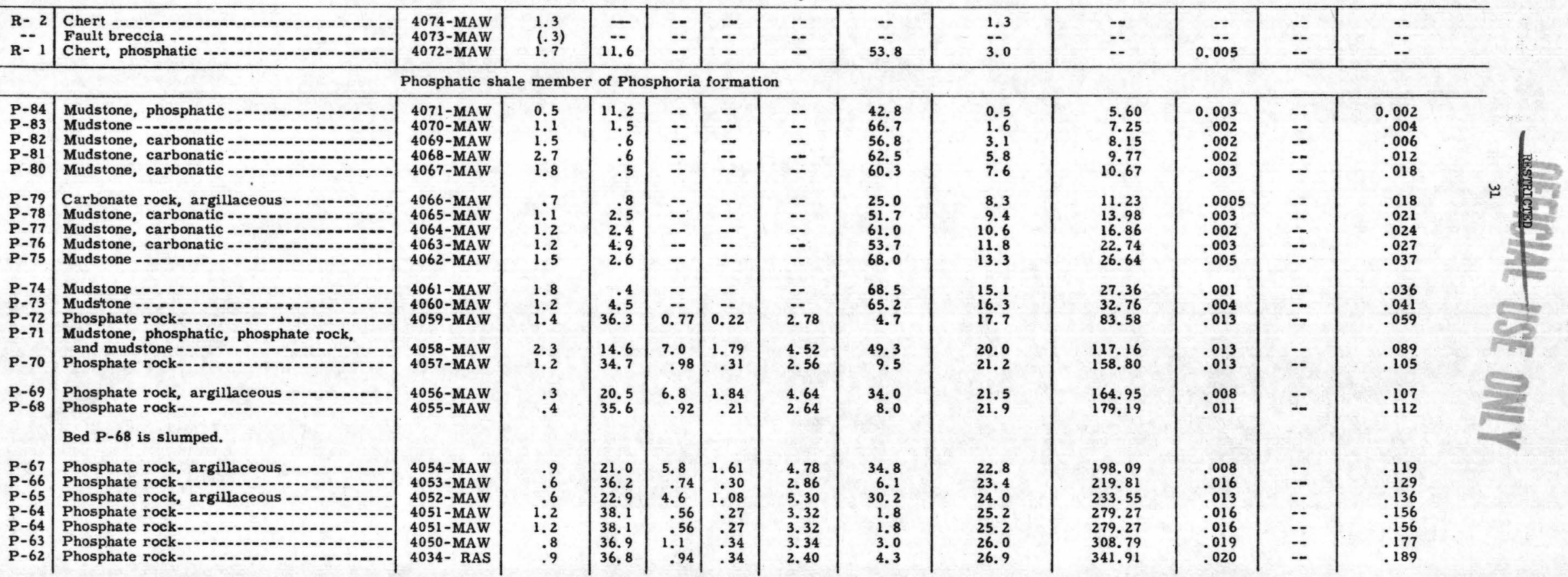


Dingle-Continued

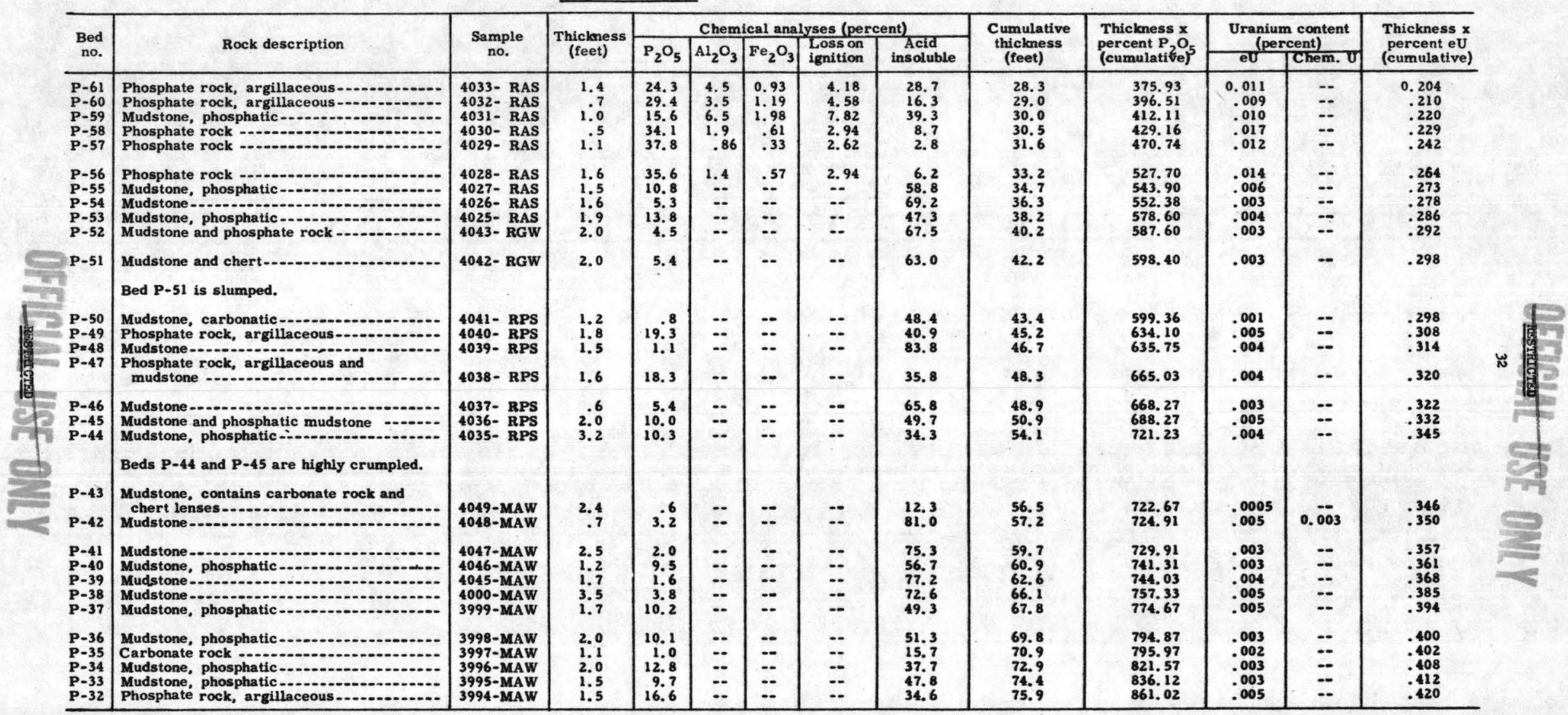



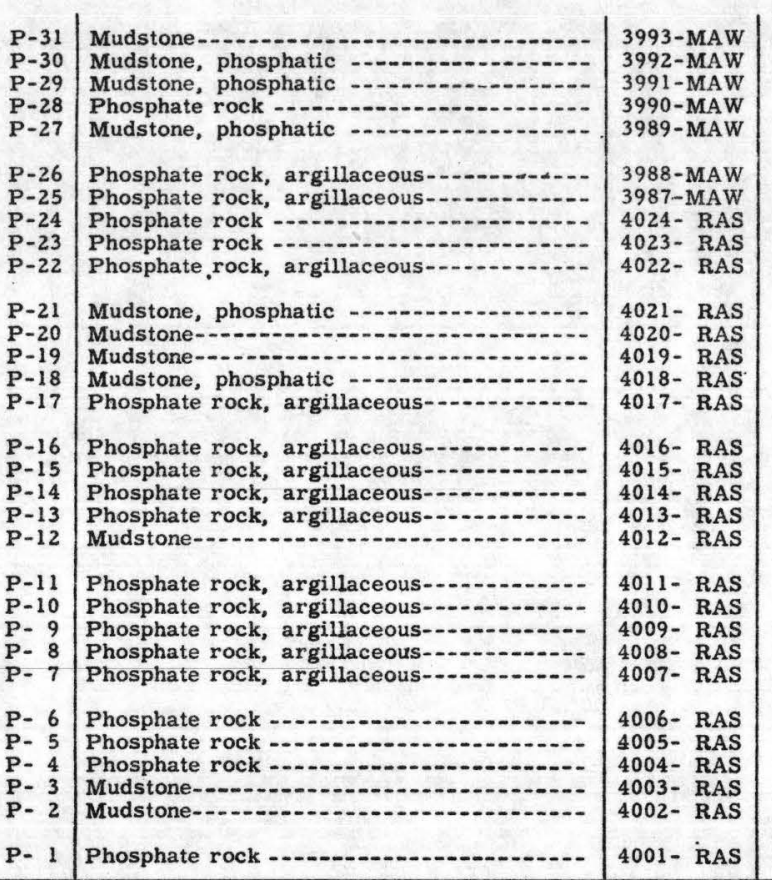

\begin{tabular}{|c|c|c|c|c|}
\hline 1.0 & 6.8 & - & $\cdots$ & -- \\
\hline 2.6 & 12. 6 & - & -- & -- \\
\hline $\begin{array}{l}2.3 \\
2.7\end{array}$ & $\begin{array}{l}12.9 \\
26.5\end{array}$ & $3 . \overline{9}$ & $1 . \overline{65}$ & 13. 34 \\
\hline 1.1 & 13.7 & 7.9 & 3.01 & 8.86 \\
\hline & 22.3 & 5.7 & 2. 00 & 9.86 \\
\hline & 18.0 & 6.7 & 2. 81 & 11.02 \\
\hline & 31.6 & $\begin{array}{l}2.2 \\
3.4\end{array}$ & $\begin{array}{l}1.00 \\
1.52\end{array}$ & 7.06 \\
\hline 2.4 & 21.6 & $\begin{array}{l}.4 \\
4.4\end{array}$ & $\begin{array}{l}1.32 \\
1.83\end{array}$ & $\begin{array}{l}4.62 \\
5.34\end{array}$ \\
\hline & 16.2 & $\ldots$ & $\ldots$ & 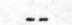 \\
\hline & 7.6 & $\because$ & - & . \\
\hline & $\begin{array}{l}4.2 \\
9.9\end{array}$ & 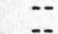 & $\because$ & -- \\
\hline 1.9 & 23.4 & 3.7 & 1.04 & 5.46 \\
\hline 1. & 28. 9 & 3.2 & .60 & 6.66 \\
\hline & $25 . ?$ & 4.1 & .70 & 4. 30 \\
\hline 0 & 24.5 & 3.9 & .94 & 5. 54 \\
\hline 2.2 & $\begin{array}{r}18.3 \\
4.7\end{array}$ & $\begin{array}{r}7.0 \\
10.0\end{array}$ & $\begin{array}{l}1.91 \\
3.50\end{array}$ & $\begin{array}{l}4.64 \\
3.92\end{array}$ \\
\hline & 26.5 & 3. 1 & 1.48 & 3.04 \\
\hline & 22. 3 & 3.6 & 6.53 & 3.64 \\
\hline & 27.4 & 4.3 & 1.34 & 3.12 \\
\hline & 22.4 & 3.6 & & 2.66 \\
\hline & 25.0 & 4.7 & 2.92 & 3.14 \\
\hline 2.8 & 35.5 & 1.3 & .34 & 3.04 \\
\hline & 35.6 & 1.3 & & 3.12 \\
\hline & 35.6 & 1.2 & .34 & 3.48 \\
\hline $\begin{array}{l}2.6 \\
1.7\end{array}$ & $\begin{array}{l}2.5 \\
1.2\end{array}$ & $\begin{array}{l}9.2 \\
9.9\end{array}$ & $\begin{array}{l}2.89 \\
3.12\end{array}$ & $\begin{array}{l}\text {, } 28 \\
3.14\end{array}$ \\
\hline .3 & 32.3 & 2.8 & 1.94 & 3.06 \\
\hline
\end{tabular}

\begin{tabular}{r|r}
65.2 & 76.9 \\
45.1 & 79.5 \\
52.1 & 81.8 \\
12.9 & 84.5 \\
46.3 & 85.6 \\
26.7 & 86.4 \\
34.8 & 87.2 \\
9.0 & 88.5 \\
16.5 & 89.4 \\
33.0 & 91.8 \\
45.8 & 93.2 \\
62.8 & 94.5 \\
70.4 & 96.7 \\
58.5 & 97.6 \\
32.5 & 99.5 \\
17.8 & 101.0 \\
27.2 & 103.4 \\
28.3 & 105.4 \\
42.0 & 107.6 \\
72.5 & 108.5 \\
26.5 & 110.5 \\
30.7 & 111.5 \\
23.0 & 113.3 \\
35.3 & 114.0 \\
27.2 & 114.9 \\
4.5 & 117.7 \\
4.2 & 119.6 \\
3.8 & 121.4 \\
77.7 & 124.0 \\
79.0 & 125.7 \\
10.5 & 126.0
\end{tabular}

867.82
900.58
930.25
$1,001.80$
$1,016.87$
$1,034.71$
$1,049.11$
$1,090.19$
$1,116.83$
$1,168.67$
$1,191.35$
$1,201.23$
$1,210.47$
$1,219.38$
$1,263.84$
$1,307.19$
$1,368.87$
$1,417.87$
$1,458.13$
$1,462.36$
$1,515.36$
$1,537.66$
$1,586.98$
$1,602.66$
$1,625.16$
$1,724.56$
$1,792.20$
$1,856.28$
$1,862.78$
$1,864.82$
$1,874.51$

.003
.004
.004
.004
.005
.004
.005
.006
.011
.010
.009
.004
.005
.004
.007
.014
.012
.009
.007
.005
.009
.004
.008
.005
.005
.009
.016
.014
.003
.003
.009

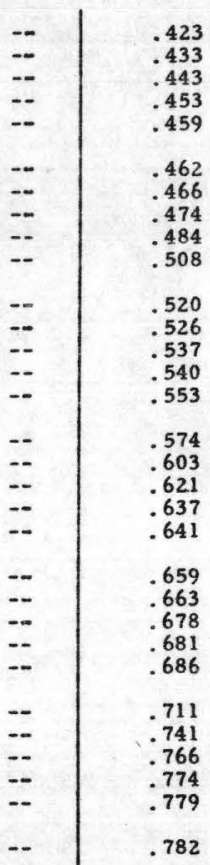

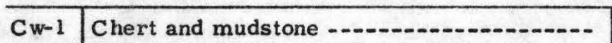

Wells formation - top part only

Upper part of the phosphatic shale member of the Phosphoria formation measured and sampled in a tunnel near the bulldozer trench. Beds strike N. $15^{\bullet} \mathrm{W}$. and dip approximately $70^{\circ}$ W. Section measured and sampled by D. F. Davidson and R. A. Smart in June 1949.

\begin{tabular}{|c|c|c|c|c|c|c|c|c|c|c|c|c|c|}
\hline$R-1$ & Chert, phosphatic -.... & 3901 - DFD & 1.1 & 10.0 & -- & -- & $-=$ & 56.4 & 1.1 & -- & 0.004 & -- & -. \\
\hline
\end{tabular}


Dingle-Continued

\begin{tabular}{|c|c|c|c|c|c|c|c|c|c|c|c|c|c|}
\hline \multirow{2}{*}{$\begin{array}{c}\text { Bed } \\
\text { no. }\end{array}$} & \multirow{2}{*}{ Rock description } & \multirow{2}{*}{$\begin{array}{c}\text { Sample } \\
\text { no. }\end{array}$} & \multirow{2}{*}{$\begin{array}{c}\text { Thickness } \\
\text { (feet) }\end{array}$} & \multicolumn{5}{|c|}{ Chemical analyses (percent) } & \multirow{2}{*}{$\begin{array}{l}\text { Cumulative } \\
\text { thickness } \\
\text { (feet) }\end{array}$} & \multirow{2}{*}{$\begin{array}{l}\text { Thickness } \mathrm{x} \\
\text { percent } \mathrm{P}_{2} \mathrm{O}_{5} \\
\text { (cumulative) }^{5}\end{array}$} & \multirow{2}{*}{\multicolumn{2}{|c|}{$\begin{array}{c}\text { Uranium content } \\
\text { (percent) }\end{array}$}} & \multirow{2}{*}{$\begin{array}{l}\text { Thickness } x \\
\text { percent eU } \\
\text { (cumulative) }\end{array}$} \\
\hline & & & & $\mathrm{P}_{2} \mathrm{O}_{5}$ & $\mathrm{Al}_{2} \mathrm{O}_{3}$ & $\mathrm{Fe}_{2} \mathrm{O}_{3}$ & $\begin{array}{l}\text { Loss on } \\
\text { ignition }\end{array}$ & $\begin{array}{c}\text { Acid } \\
\text { insoluble }\end{array}$ & & & & & \\
\hline \multicolumn{14}{|c|}{ Phosphatic shale member of Phosphoria formation } \\
\hline $\mathrm{P}-33$ & Mudstone, phosphatic & 3902-DFD & 0.5 & 10.6 & -- & -- & -- & 55.0 & 0.5 & 5.30 & 0.007 & -- & 0.004 \\
\hline $\begin{array}{l}P-32 \\
P-31\end{array}$ & Mudstone & 3903-DFD & .9 & 2.0 & -- & -- & $=$ & 73.9 & 1.4 & 7.10 & .002 & -- & .005 \\
\hline $\begin{array}{l}P-31 \\
P-30\end{array}$ & $\begin{array}{l}\text { Mudstone } \\
\text { Mudstone, carbonatic }\end{array}$ & $\begin{array}{l}\text { 3904-DFD } \\
\text { 3905-DFD }\end{array}$ & 2.7 & $\begin{array}{r}3.5 \\
.3\end{array}$ & $\overline{--}$ & 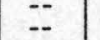 & $=$ & $\begin{array}{l}67.0 \\
60.0\end{array}$ & $\begin{array}{l}2.1 \\
4.1\end{array}$ & $\begin{array}{r}9.55 \\
10.15\end{array}$ & .004 & $\therefore$ & .008 \\
\hline$P-29$ & Mudstone, carbonatic - & 3906-DFD & 3.4 & .5 & -- & -- & -- & 51.0 & 7.5 & 11.85 & .003 & - & .020 \\
\hline -- & Mudstone, carbonatic lens in bed P-29....- & 3907-DFD & -- & .2 & -- & -- & -- & 60.1 & -- & -- & .002 & -- & -- \\
\hline $\mathrm{P}-28$ & Mudstone, carbonatic & 3908-RAS & 2.1 & 1.5 & -- & -- & -- & 61.3 & 9.6 & 15.00 & .002 & -- & .024 \\
\hline $\mathrm{P}-27$ & Carbonate rock, argillaceous & 3909-RAS & .7 & .8 & -- & -- & -- & 26.7 & 10.3 & 15.56 & .001 & -- & .025 \\
\hline$P-26$ & $\begin{array}{l}\text { Mudstone } \\
\text { Phosphate rock, argillaceous }\end{array}$ & 4080-RAS & $\begin{array}{r}2.0 \\
2.0\end{array}$ & 6.3 & -- & $\overline{-}$ & 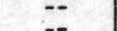 & 54.3 & 12.3 & 28. 16 & .004 & -- & .033 \\
\hline$P-25$ & & 4081-RAS & .8 & 17.9 & -- & -- & -- & 41.8 & 13.1 & 42.48 & .005 & $\cdots$ & .037 \\
\hline P-24 & Mudstone - & 4082-RAS & 1.8 & 3.4 & -- & -- & -- & 69.5 & 14.9 & 48.60 & .004 & -- & .044 \\
\hline $\begin{array}{l}P-23 \\
P-22\end{array}$ & $\begin{array}{l}\text { Mudstone } \\
\text { Phosphate rock }\end{array}$ & $\begin{array}{l}\text { 4083-RAS } \\
\text { 4084-RAS }\end{array}$ & $\begin{array}{l}2.4 \\
1.7\end{array}$ & 33.8 & $\overline{1.6}$ & $0 . \overline{47}$ & 2.56 & $\begin{array}{r}73.7 \\
9.5\end{array}$ & $\begin{array}{l}17.3 \\
19.0\end{array}$ & $\begin{array}{r}49.80 \\
107.26\end{array}$ & .002 & $\therefore$ & .049 \\
\hline$P-21$ & Mudstone and argillaceous phosphate & & & & & & & & & & & & \\
\hline P-20 & $\begin{array}{l}\text { rock }-1,0 \\
\text { Mudstone - }\end{array}$ & $\begin{array}{l}\text { 4085-RAS } \\
\text { 4086-RAS }\end{array}$ & $\begin{array}{l}.8 \\
1.0\end{array}$ & $\begin{array}{l}8.7 \\
5.0\end{array}$ & $\begin{array}{l}9.1 \\
9.6\end{array}$ & $\begin{array}{l}1.63 \\
1.69\end{array}$ & $\begin{array}{l}3.86 \\
3.90\end{array}$ & $\begin{array}{l}62.4 \\
72.7\end{array}$ & $\begin{array}{l}19.8 \\
20.8\end{array}$ & $\begin{array}{l}114.22 \\
119.22\end{array}$ & .003 & $\overline{-}$ & .074 \\
\hline$P-19$ & Phosphate rock & 4087-RAS & .4 & 35.6 & 1,5 & .43 & 2.32 & 6.4 & 21,2 & 133,46 & .012 & -- & .082 \\
\hline $\mathrm{P}-18$ & Phosphate rock and phosphatic mudstone -.. & 4088-RAS & 1.0 & 23.5 & 7.0 & 1.22 & 3.80 & 30.3 & 22.2 & 156.96 & .008 & -. & .090 \\
\hline $\mathrm{P}-17$ & Phosphate rock & 4089-RAS & 1.1 & 34.4 & 1.3 & .34 & 1.96 & 9.4 & 23.3 & 194.80 & .012 & -- & .104 \\
\hline P-16 & Phosphate rock & 4090-RAS & .7 & 33.3 & 2.8 & .67 & 2.76 & 11.1 & 24.0 & 218.11 & .011 & -- & .111 \\
\hline$P-15$ & $\begin{array}{l}\text { Phosphate rock, argillaceous and } \\
\text { phosphate rock }\end{array}$ & 4091-RAS & 1.0 & 25.8 & 4.8 & 1.74 & 6.40 & 22.7 & 25.0 & 243.91 & .010 & -- & .121 \\
\hline P-14 & Phosphate rock - & 4092-RAS & .9 & 35.5 & 1.1 & .64 & 5. 28 & 4.3 & 25.9 & 275.86 & .018 & -- & .138 \\
\hline $\mathrm{P}-13$ & Mudstone, phosphatic and phosphate rock -- & 4093-RAS & .8 & 26.6 & 4.1 & 1.88 & 6.62 & 20.5 & 26.7 & 297.14 & .012 & -- & .147 \\
\hline $\mathrm{P}-12$ & & 4094-RAS & 1.9 & 36.9 & .73 & .38 & 5.66 & 1.7 & 28.6 & 367.25 & .014 & 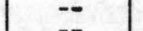 & .174 \\
\hline $\begin{array}{l}P-11 \\
P-10\end{array}$ & Phosphate rock & 4095-RAS & 2.1 & 35.3 & 1.2 & .84 & 6.08 & 3.4 & 30.7 & $\begin{array}{l}441.38 \\
477.08\end{array}$ & .019 & $=$ & .2214 \\
\hline$P=10$ & Phosphate rock, argillaceous & 4096-RAS & 1.5 & 23.8 & 5.8 & & 7. 88 & & & 477.08 & & $\cdots$ & \\
\hline P- 9 & Phosphate rock & 4097-RAS & 1.9 & 36.9 & 1.4 & .61 & 3.14 & 2.7 & 34. 1 & $\begin{array}{l}547.19 \\
582.89\end{array}$ & .018 & $=$ & .257 \\
\hline $\begin{array}{l}\text { P- } \\
P-7\end{array}$ & $\begin{array}{l}\text { Phosphate rock } \\
\text { Phosphate rock, argillaceous }\end{array}$ & $\begin{array}{l}\text { 4098-RAS } \\
\text { 4099-RAS }\end{array}$ & $\begin{array}{l}1.0 \\
1.6\end{array}$ & $\begin{array}{l}35.7 \\
24.4\end{array}$ & $\begin{array}{l}1.4 \\
4.0\end{array}$ & $\begin{array}{l}.71 \\
1.77\end{array}$ & $\begin{array}{r}3.94 \\
11.62\end{array}$ & 19.5 & 36. 7 & $\begin{array}{l}582.89 \\
621.93\end{array}$ & .022 & - & .295 \\
\hline P- 6 & Mudstone, phosphatic - & 5000-RAS & 1.2 & 11.7 & $=$ & -2 & -- & 52.0 & 37.9 & 635.97 & .004 & -- & .300 \\
\hline P- 5 & Mudstone & 5001-RAS & 1.5 & 5.4 & -- & -- & -- & 66.1 & 39.4 & 644.07 & .004 & - & .306 \\
\hline P- 4 & Mudstone & 5002-RAS & 2.6 & 1.7 & -- & -- & -- & 80.3 & 42.0 & 648.49 & .003 & -- & .314 \\
\hline P- 3 & Mudstone & 5003-RAS & 2.0 & 4. 9 & -- & -- & -- & 71.8 & 44.0 & 658.29 & .002 & -- & .318 \\
\hline P- 2 & Phosphate rock & 5004-RAS & .4 & 28.6 & $-\infty$ & -- & $=$ & 13.4 & 44.4 & $\begin{array}{r}669.73 \\
672.28\end{array}$ & .005 & $\because$ & .320 \\
\hline P- 1 & Mudstone & 5005-RAS & .5 & 5.1 & $-\infty$ & -- & -- & 56.0 & 44.9 & 672.28 & .005 & -- & .322 \\
\hline
\end{tabular}

lot 1293

IV - Idaho - 24 
Hot Springs, Idaho, lot 1317

Portion of the phosphatic shale member of the Phosphoria formation sampled on overturned east limb of Hot Springs anticline in the Hot Springs crosscut, sec. 13, T. 15 S., R. 44 E., Bear Lake County, Idaho. Beds strike N. $24^{\circ} \mathrm{W}$. and dip $43^{\circ} \mathrm{W}$. Section measured by R. G. Waring, J. D. Weiser, H. W. Peirce and sampled by Waring and Peirce in September 1949. Samples analyzed for $\mathrm{P}_{2} \mathrm{O}_{5}$ and acid insoluble by U. S. Bureau of Mines
laboratory, Albany, Oreg., and for other constituents by Trace Elements Section laboratory, ${ }^{\mathrm{U}}$. S. Geological Survey, Washington, D. C.
Samples analyzed for $\mathrm{eU}$ and chem. U by the U. S. Geological Surve laboratory, Geochemistry and Petrology Branch.

\begin{tabular}{|c|c|c|c|c|c|c|c|c|c|c|c|c|}
\hline \multirow{2}{*}{$\begin{array}{c}\text { Bed } \\
\text { no. }\end{array}$} & \multirow[b]{2}{*}{ Rock description } & \multirow{2}{*}{$\begin{array}{c}\text { Sample } \\
\text { no. }\end{array}$} & \multirow{2}{*}{$\begin{array}{c}\text { Thickness } \\
\text { (feet) }\end{array}$} & \multicolumn{5}{|c|}{ Chemical analyses (percent) } & \multirow{2}{*}{$\begin{array}{l}\text { Cumulative } \\
\text { thickness } \\
\text { (feet) }\end{array}$} & \multirow{2}{*}{$\begin{array}{l}\text { Thickness } \mathrm{x} \\
\text { percent } \mathrm{P}_{2} \mathrm{O}_{5} \\
\text { (cumulative) }\end{array}$} & \multirow{2}{*}{$\begin{array}{c}\begin{array}{c}\text { Uranium content } \\
\text { (percent) }\end{array} \\
\text { eU Chem. U }\end{array}$} & \multirow{2}{*}{$\begin{array}{l}\text { Thickness } x \\
\text { percent chem, } \\
\text { (cumulative) }\end{array}$} \\
\hline & & & & $\mathrm{P}_{2} \mathrm{O}_{5}$ & $\mathrm{Al}_{2} \mathrm{O}_{3}$ & $\mathrm{Fe}_{2} \mathrm{O}_{3}$ & \begin{tabular}{|l|} 
Loss on \\
ignition
\end{tabular} & $\begin{array}{c}\text { Acid } \\
\text { insoluble }\end{array}$ & & & & \\
\hline
\end{tabular}

Upper portion of phosphatic shale member of Phosphoria formation

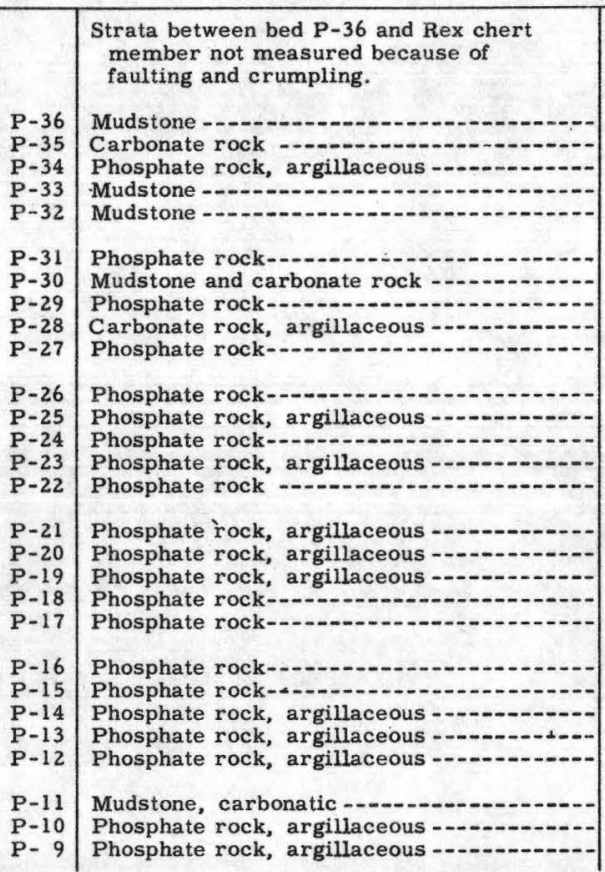

lot 1317
4615- JDW 4614- JDW 4613- JDW 4612-JDW 4610- JDW 4609- JDW 4608-JDW 4607- JDW 4606-JDW 4605-HWP 4604-HWP 4603-HWP $4602-\mathrm{HWP}$
$4601-\mathrm{HWP}$

4600-HWP 4599-HWP 4597-HW 4596-HWP 4595-HWP 4594-HWP 4592-HWP 4591-HWP 4590-HWP 4589-HWP 4588-HWP

\begin{tabular}{|c|c|c|c|c|}
\hline 1.3 & 2.3 & -- & - & -- \\
\hline 5.0 & & 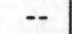 & 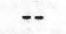 & 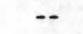 \\
\hline .4 & 24.6 & -- & -- & $=-$ \\
\hline $\begin{array}{l}1.3 \\
3.0\end{array}$ & $\begin{array}{l}3.2 \\
1.0\end{array}$ & $-\infty$ & $=$ & $=$ \\
\hline 2.0 & 34.3 & 0.56 & 0.39 & 6.17 \\
\hline 2.0 & 4.1 & 4.96 & 1.75 & 21.63 \\
\hline .5 & 30.0 & .90 & .61 & 9.48 \\
\hline 1.3 & 5.5 & 4.72 & 1.86 & 23.15 \\
\hline .8 & 31.2 & .70 & .36 & 6.58 \\
\hline 1.3 & 31.0 & 1.62 & .84 & 7.50 \\
\hline & 22.8 & 4.16 & 1.75 & 7.55 \\
\hline & 31.7 & 1.27 & .63 & 7.01 \\
\hline & 19.5 & 3.84 & 1.75 & 12.85 \\
\hline .8 & 33.8 & 1.00 & .63 & 9.38 \\
\hline 8 & 24.1 & 2.78 & 1.26 & 11.42 \\
\hline .7 & 24.4 & 2.60 & 1.12 & 13.30 \\
\hline 1.4 & 22.4 & 4.60 & 1.80 & 11.43 \\
\hline & 32.4 & .62 & .46 & 8.83 \\
\hline 1 & 25.4 & 1.10 & .48 & 9.83 \\
\hline 1.6 & 32.0 & 2. 19 & 1.06 & 15.73 \\
\hline 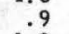 & 30.2 & 1. 21 & .56 & 14.32 \\
\hline 19 & 28.1 & 3.16 & 1.36 & 14.16 \\
\hline 1.3 & 18.6 & & & \\
\hline 2.7 & 15.7 & -- & -- & -- \\
\hline .7 & 5.7 & -- & -- & - \\
\hline $\begin{array}{r}1.4 \\
.6\end{array}$ & $\begin{array}{l}16.7 \\
16.5\end{array}$ & -- & -- & - \\
\hline
\end{tabular}

67.2
16.6
21.1
65.9
72.8
2.4
36.4
4.9
30.0
3.6
7.9
26.0
7.8
24.6
3.6
16.9
15.6
27.1
2.6
4.3
10.6
5.0
17.9
19.9
37.7
40.2
35.0
35.4

0.003 \begin{tabular}{l|l}
1.3 & 2.99 \\
6.3 & 4.99
\end{tabular}

6.3
6.7
8.0
11.0
13.0
15.0
15.5
16.8
17.6
18.9
19.7
20.5
21.25
22.05
22.85
23.55
24.95
25.75
27.85
29.45
30.35
32.25
33.75
36.45
39.15
40.55
41.15

4.99

14.83
18.99

21.99

90.59

98.79
113.79

120.94

186. 20

204.44

229.80
244.43

271.47

290.75
307.83

365.1

418.45

469.65

496.83

578.12

578.12
620.51

635.90

659.28
669.18 
Hot Springs - Continued

\begin{tabular}{|c|c|c|c|c|c|c|c|c|c|c|c|c|c|}
\hline \multirow{2}{*}{$\begin{array}{r}\text { Bed } \\
\text { no. }\end{array}$} & \multirow{2}{*}{ Rock description } & \multirow{2}{*}{$\begin{array}{l}\text { Sample } \\
\text { no. }\end{array}$} & \multirow{2}{*}{$\begin{array}{c}\text { Thickness } \\
\text { (feet) }\end{array}$} & \multicolumn{5}{|c|}{ Chemical analyses (percent) } & \multirow{2}{*}{$\begin{array}{l}\text { Cumulative } \\
\text { thickness } \\
\text { (feet) }\end{array}$} & \multirow{2}{*}{$\begin{array}{l}\text { Thickness } x \\
\text { percent } P_{2} O_{5} \\
\text { (cumulative) }\end{array}$} & \multirow{2}{*}{\multicolumn{2}{|c|}{$\begin{array}{c}\text { Uranium content } \\
\text { (percent) }\end{array}$}} & \multirow{2}{*}{$\begin{array}{l}\text { Thickness } x \\
\text { percent chem. } \\
\text { (cumulative) }\end{array}$} \\
\hline & & & & $\mathrm{P}_{2} \mathrm{O}_{5}$ & $\mathrm{Al}_{2} \mathrm{O}_{3}$ & $\mathrm{Fe}_{2} \mathrm{O}_{3}$ & \begin{tabular}{|l} 
Loss on \\
ignition
\end{tabular} & $\begin{array}{l}\text { Acid } \\
\text { insoluble }\end{array}$ & & & & & \\
\hline $\begin{array}{l}P-8 \\
P-7 \\
P-6 \\
P-5 \\
P-4\end{array}$ & $\begin{array}{l}\text { Mudstone, phosphatic } \\
\text { Mudstone, phosphatic } \\
\text { Mudstone - } \\
\text { Phosphate rock, argillaceous } \\
\text { Mudstone, phosphatic }\end{array}$ & $\begin{array}{l}\text { 4587-HWP } \\
4586-\text { HWP } \\
4585-\text { HWP } \\
4584-\text { HWP } \\
4583-\text { HWP }\end{array}$ & $\begin{array}{l}2.2 \\
1.5 \\
1.4 \\
.7 \\
2.3\end{array}$ & $\begin{array}{r}12.9 \\
13.0 \\
1.7 \\
20.7 \\
11.5\end{array}$ & $\begin{array}{l}-: \\
-- \\
-- \\
-\end{array}$ & $\begin{array}{l}\because- \\
\because- \\
--\end{array}$ & $\begin{array}{l}= \\
= \\
- \\
-\end{array}$ & $\begin{array}{l}42.6 \\
47.6 \\
72.0 \\
32.5 \\
51.8\end{array}$ & $\begin{array}{l}43.35 \\
44.85 \\
46.25 \\
46.95 \\
49.25\end{array}$ & $\begin{array}{l}697.56 \\
717.06 \\
719.44 \\
733.93 \\
760.38\end{array}$ & $\begin{array}{l}0.004 \\
.004 \\
.004 \\
.005 \\
.004\end{array}$ & $\begin{array}{r}0.004 \\
.004 \\
.001 \\
.005 \\
.004\end{array}$ & $\begin{array}{r}0.361 \\
.367 \\
.369 \\
.372 \\
.381\end{array}$ \\
\hline $\begin{array}{l}P-3 \\
P-2 \\
P-1\end{array}$ & $\begin{array}{l}\text { Carbonate rock } \\
\text { Mudstone, phosphatic } \\
\text { Phosphate rock, argillaceous }\end{array}$ & $\begin{array}{l}4582-\mathrm{HWP} \\
4581-\mathrm{HWP} \\
4580-\mathrm{HWP}\end{array}$ & $\begin{array}{l}1.3 \\
2.2 \\
1.2\end{array}$ & $\begin{array}{r}1.3 \\
27.0 \\
16.9\end{array}$ & $\begin{array}{l}-- \\
--\end{array}$ & $\begin{array}{l}-- \\
--\end{array}$ & $\begin{array}{l}-- \\
--\end{array}$ & $\begin{array}{l}13.8 \\
26.4 \\
31.6\end{array}$ & $\begin{array}{l}50.55 \\
52.75 \\
53.95\end{array}$ & $\begin{array}{l}762.07 \\
821.46 \\
841.74\end{array}$ & $\begin{array}{l}.001 \\
.004 \\
.005\end{array}$ & $\begin{array}{l}.001 \\
.003 \\
.006\end{array}$ & $\begin{array}{l}.383 \\
.389 \\
.396\end{array}$ \\
\hline
\end{tabular}

A fault at base of bed P-1 causes omission

of an unknown thickness of strata. 